\title{
Exact and Approximate Theoretical Techniques for Quantum Magnetism in Low Dimensions
}

\author{
Swapan K. Pati ${ }^{1}$, S. Ramasesha ${ }^{2}$, Diptiman Sen $^{3}$ \\ ${ }^{1}$ Jawaharlal Nehru Centre for Advanced Scientific Research, \\ Jakkur, Bangalore 560064, India \\ 2 Solid State and Structural Chemistry Unit, \\ Indian Institute of Science, Bangalore 560012, India \\ ${ }^{3}$ Centre for Theoretical Studies, \\ Indian Institute of Science, Bangalore 560012, India
}

\begin{abstract}
Quantum magnetism in low dimensions has been one of the central areas of theoretical research for many decades now. One of the key reasons for the long standing interest in this field has been the existence of simplified models, which serve as paradigms for understanding the role of strong interactions in many-electron systems. Although simple, these models quite often can not be solved exactly. In this review, we discuss a variety of analytical and numerical methods, which treat the system in a systematic and controlled manner. The central method employed in all the studies is the density matrix renormalization group (DMRG) method. This is supported by small scale numerical exact calculations, and by analytical methods using field theoretic techniques. We have considered a number of magnetic systems, from magnetic clusters to extended lattices, and have found some novel quantum ground states and low-energy elementary excitations. In some cases, we have also employed the finite-temperature DMRG method to accurately compute the low-temperature thermodynamic properties such as specific heat and magnetic susceptibility.
\end{abstract}




\section{Introduction}

The nonrelativistic Schrödinger equation of a system of electrons is spin independent. It therefore appears at first glance that the solutions of the Schrödinger equation should also be spin independent. However, the indistinguishability of the electrons forces the total wave function which is a product of the spin wave function and the spatial wave function to be antisymmetric. This in turn implies that for two electrons, a spatially symmetric wave function should be associated with an antisymmetric spin wave function and vice versa. The different charge distribution in the spatially symmetric and antisymmetric wave functions leads to different coulomb repulsions by virtue of which the spin states which are symmetric and antisymmetric have different energies [1]. Dirac represented the splitting between the energies of the two spin states by the spin operator $-2 J \hat{\mathbf{S}}_{i} \cdot \hat{\mathbf{S}}_{j}$, where $J$ is the exchange integral involving the two spatial orbitals in which the two electrons are singly occupied. In most open shell atomic systems the exchange integral $J$ is large enough to force the total spin of the ground state configuration to be the largest permissible value. This in essence is the Hund's rule of maximum multiplicity and is also the reason why we find transition and rare earth metal ions in the high spin states in nature.

In solids containing transition metal or rare earth ions surrounded by ligands, the relative alignment of the unpaired spins at the metal site is not at all obvious. In order to understand this, we should examine the possible pathways for the delocalization of the valence electrons in the system. If the favorable delocalization pathways involve antiparallel alignment of the metal ion spins then the nature of the exchange interaction between the metal ion spins is antiferromagnetic and ferromagnetic otherwise. This follows from the fact that delocalization of electrons lowers kinetic energy of the electrons and therefore the ground state corresponds to an alignment of spins that allows for maximum delocalization. This is indeed the reason why the ground state of a hydrogen molecule is a spin singlet. In a system with degenerate partially occupied orbitals, the Hund's coupling favors high-spin alignment of the electrons on an ion. If delocalization pathways exist that allow for these high-spin states in the process of delocalization, then the alignment of the spins on two neighboring centers will be ferromagnetic. If delocalization pathways exist only when these high-spin states are aligned antiparallel, one would have an antiferromagnetic alignment of the spin. Thus, the overall nature of the spin alignment is governed by a competition between the Hund's coupling and electron delocalization [2, 3].

Given a collection of spins, the exchange Hamiltonian for the system is written 
as

$$
H=\sum_{i j} J_{i j} \hat{\mathbf{S}}_{i} \cdot \hat{\mathbf{S}}_{j}
$$

where $J_{i j}$ is the effective exchange integral for the interaction between the spins at sites ' $i$ ' and ' $j$ '. Since the spins in the cluster arise from unpaired electrons of a transition metal ion in a crystal field, it is natural to expect that the spin-orbit as well as spin-spin interactions of the electrons in the ion could alter the nature of the total spin on the ion by giving the net spin a preferred direction of orientation. Such a situation can be easily handled by treating each $J_{i j}$ as a vector and generalizing the exchange Hamiltonian as

$$
H=\sum_{i j}\left(J_{i j}^{x} \hat{S}_{i}^{x} \hat{S}_{j}^{x}+J_{i j}^{y} \hat{S}_{i}^{y} \hat{S}_{j}^{y}+J_{i j}^{z} \hat{S}_{i}^{z} \hat{S}_{j}^{z}\right)
$$

Such a model is often referred to as the XYZ spin model [2]. Two extreme cases are often studied, (i) the spin is assumed to have no projection on the X-Y plane, in which case the resulting model is the Ising model and corresponds to scalar spins, and (ii) the spin is assumed to have no projection on the z-axis, in which case we have an XY model or a planar spin model. The Ising model is a discrete classical model as it consists of no noncommuting operators in its Hamiltonian while the XY model could be classical or quantum mechanical. Usually, while dealing with large site spin systems, it is not uncommon to assume that the spins are classical, in the spirit of Bohr's correspondence principle.

In the crystalline state, the spins in the solid would be arranged on a lattice. If the exchange interaction is predominant between spins along a single crystalline direction, the model could be treated as a one dimensional array of spins. There are many examples of solids in which this holds [4]. Likewise, it is also possible that the interactions amongst spins is large along two crystallographic directions and weak along a third direction and this would result in two-dimensional spin system [5].

In this review article, we will mainly concern ourselves with the study of isotropic spin clusters and one-dimensional spin systems, sometimes in the presence of an external magnetic field. We will be mostly interested in properties of the ground state and the low-lying excitations, since these are the states which govern the low-temperature properties of systems such as the specific heat and magnetic susceptibility. The symmetries of a system often enable us to characterize the energy eigenstates in terms of quantum numbers such as the total spin $S_{t o t}$, the component of the total spin along some particular direction, say, $S_{t o t, z}$, spin parity (which is a symmetry for states with $S_{t o t, z}=0$ ), the wave number $k$ for a translation invariant system, and possibly other spatial symmetries depending on the structure. We will 
discuss below how the use of symmetries can help to lessen the numerical effort required to study the low-energy states.

In the next two sections we introduce some exact numerical methods, and describe the application of these methods to magnetic clusters. In section 4, we discuss two analytical methods which use field theoretic approximations. In section 5, we describe an innovative way of solving the spin Hamiltonians, which goes beyond the conventional techniques and is based on the density matrix renormalization group (DMRG) theory. Various applications of DMRG to the properties of low-dimensional extended chains are described in sections 6 to 8 .

\section{Exact Calculations}

The properties of a spin Hamiltonian can be computed from the eigenstates of the Hamiltonian which are in turn obtained by setting up the Hamiltonian matrix in a suitable basis and diagonalizing it thereafter. While the procedure itself is quite straightforward, the space spanned by the Hamiltonian rapidly increases with the number of the spins in the system. The Fock space dimensionality of a system of $n$ spins with spin $s_{i}$ is given by

$$
D_{F}=\prod_{i=1}^{n}\left(2 s_{i}+1\right) .
$$

The Hamiltonian matrix is block-diagonal in structure with each block corresponding to specified values of the quantities conserved by the Hamiltonian. Thus, for an isotropic spin system, the $z$-component of the total spin, $M_{S}$, and the total spin $S$ are conserved. Restricting the Fock space to specified values of $M_{S}$ and $S$ gives Hilbert spaces whose dimensionalities are smaller than the Fock space dimensionality.

While constructing spin basis functions which are eigenstates of the total $\hat{S}^{z}$ operator is quite simple, construction of spin adapted functions (SAF, eigenstates of

the total $\hat{\mathbf{S}}^{2}$ operator) is not direct. Perhaps the simplest and chemically appealing way of constructing SAF is by the valence bond (VB) method which uses the RumerPauling rules. This method is best illustrated by applying it to a system of $2 n$ spins, each possessing a spin of half, in the total spin $S=0$ sector. A total spin singlet can be formed by choosing pairs of sites and spin coupling each of them to obtain a singlet. The product of these singlet pairs will be a spin eigenstate of the operator $\hat{\mathbf{S}}_{\text {total }}^{2}$. This is illustrated in Fig. 1. However, there are more ways to spin couple in pairs than the number of linearly independent singlet states, e.g., the state $\mid 3>$ in Fig. 1 can be expressed as a linear combination of the states $\mid 1>$ and $\mid 2>$. 
The overcompleteness can be avoided by resorting to the Rumer-Pauling rules. To implement this rule, we arrange the $2 n$ spins at the vertices of a regular $2 n$-gon. We draw lines between pairs of sites that are singlet paired. According to the RumerPauling rule, the subset of these, encompassing all diagrams (to be called 'legal' diagrams) with no crossing lines, forms a complete and linearly independent set of states [6].

The Rumer-Pauling rules can be easily extended to construct complete and linearly independent basis sets in higher spin Hilbert spaces involving spin- $1 / 2$ objects. This is done most easily with the help of phantom sites. If we wish to construct VB diagrams for total spin $S$ subspace involving $n$ spin-1/2 objects, then we introduce $2 S$ additional sites to be called phantom sites. Besides imposing the Rumer-Pauling rules on the diagrams with $n+2 S$ sites, we impose the additional constraint that there should be no singlet lines amongst the $2 S$ phantom sites. In Fig. 2, we show a few examples of VB diagrams with higher total spin.

It is also quite simple to extend the VB rules to spin clusters made up of different site spins [0]. If the spin at a site is $s_{i}$, then we replace this site by a set of $2 s_{i}$ sites, each with spin- $1 / 2$. We then proceed with constructing the VB basis, as though the system is made up entirely of spin- $1 / 2$ objects, with one difference, namely, we impose the additional constraint that there should be no singlet lines within the subset of $2 s_{i}$ sites which replace the spin $s_{i}$ at site $i$. The VB diagrams with total spin $S \geq \frac{1}{2}$ are constructed as before with the help of phantom sites. An example of a legal VB diagram involving higher site spins is shown in Fig. 2.

Generating and storing the VB diagrams on a computer is also quite simple. We associate one bit with every site. The state of the bit is ' 1 ', if in the VB diagram a line begins at the site and the state is ' 0 ' if a line ends at the site. Thus, we can associate an integer of $n$ bits with every VB diagram involving $n$ spin- $1 / 2$ objects. This association is unique if we decipher the bit pattern of the integer corresponding to the diagram from inside-out, much like expanding an algebraic expression with multiple parentheses. In Fig. 2, we have also shown the bit pattern and the associated integer for each VB diagram. The VB diagrams are generated on a computer by checking the bit pattern in all $n$-bit integers to see if they satisfy the criterion for representing the desired VB diagram. This also allows us to generate the VB diagrams as an ordered sequence of the integers that represent them, a fact that helps in rapid generation of the Hamiltonian matrix.

The Hamiltonian matrix in the VB basis can be easily constructed by knowing the action of the operator $\hat{\mathbf{S}}_{i} \cdot \hat{\mathbf{S}}_{j}$ for spin-1/2 particles (i) on a singlet line joining sites $i$ and $j$ and (ii) on the pair of sites $i$ and $j$ singlet paired to two different sites $i^{\prime}$ and $j^{\prime}$ (Fig. 3). In the case of Hilbert spaces with nonzero total $S$, the Hamiltonian involves 
spin exchange between the real sites only. However, these exchange operators could lead to VB diagrams in which the phantom sites are interconnected. In this event, simply neglecting these resultant states is sufficient to ensure that we are dealing exactly with the spin $S$ Hilbert space. The Hamiltonian for spin clusters with arbitrary spins can be treated as consisting of operators with only spin- $1 / 2$ objects. This is done by replacing the spin exchange operator between sites $i$ and $j, \hat{\mathbf{S}}_{i} \cdot \hat{\mathbf{S}}_{j}$ by the operator $\left(\Sigma_{k=1}^{2 s_{i}} \hat{\tau}_{k}\right) \cdot\left(\Sigma_{l=1}^{2 s_{j}} \hat{\tau}_{l}\right)$, where the operators $\hat{\tau}_{k}$ and $\hat{\tau}_{l}$ are the usual spin-1/2 operators.

The matrix representing the Hamiltonian in the VB basis is in general nonsymmetric since the VB basis is nonorthogonal. However, the matrix itself is sparse. There exist efficient numerical algorithms [8] for obtaining the low-lying eigenstates of sparse nonsymmetric matrices, and it is possible to solve a nonsymmetric matrix eigenvalue problem for a million by million matrix with about a 100 million nonzero matrix elements on a powerful PC based workstation.

While VB theory guarantees spin purity in the computed eigenstates of the spin conserving Hamiltonians, it has several drawbacks. Computation of quantities such as spin-spin correlation functions and spin densities are not easy because a site-spin operator operating on a VB diagram spoils the spin purity of the diagram. This could be overcome by converting the eigenstate of the Hamiltonian in the VB basis to the constant $M_{S}$ basis. Another difficulty with the VB procedure is exploiting the spatial symmetries of the problem. Operation by a spatial symmetry operator on a legal VB diagram could lead to illegal VB diagrams. Disentangling these illegal diagrams into legal diagrams can be computationally prohibitive [9].

In more general spin problems, it is often advantageous to use the constant $M_{S}$ basis and exploit all the spatial symmetries. Partial spin symmetry adaptation in these cases is also possible by using the spin parity operator. The effect of the spin parity operator on a basis state is to flip all the spins in the state. In the $M_{S}=0$ sector, it is possible to factor the Hilbert space into odd and even parity Hilbert spaces. The odd (even) parity Hilbert space is spanned by basis vectors with odd (even) total spin. This also has the effect of reducing the dimensionality of the Hilbert space besides providing partial spin symmetry adaptation. It is rather simple to set up the Hamiltonian matrix in the symmetry adapted basis. The Hamiltonian matrix is symmetric and usually very sparse. The lowest few eigenstates can be easily computed by employing the Davidson algorithm. Given these eigenstates, the computation of properties can proceed by converting an eigenstate in the symmetrized basis into that in the unsymmetrized basis. The orthogonality of the basis states as well as the simple rules involved in obtaining the resultant when a basis state is operated upon by any type of spin operators in any combination affords easy 
computation of a variety of properties of a magnetic system.

The exact diagonalization techniques discussed above are in general applicable to systems whose Hilbert space dimensionality is about 10 million. The major problem with exact diagonalization methods is the exponential increase in dimensionality of the Hilbert space with the increase in the system size. Thus, the study of larger systems becomes not only CPU intensive but also memory intensive as the number of nonzero elements of the matrix increases rapidly with system size. With increasing power of the computers, slightly larger problems have been solved every few years. To illustrate this trend, we consider the case of the spin-1 Heisenberg chain. In 1973, ten years before the Haldane conjecture, De Neef [10] used the exact diagonalization procedure to solve a 8 -site spin-1/2 chain. In 1977, Blote [11 diagonalized the Hamiltonian of a chain of 10 sites. In 1982, Botet and Jullien [12] increased this to 12 sites. In 1984, Parkinson and Bonner 13 solved the 14-site spin-1 problem. In the same year, Moreo [14] solved the 16-site spin-1 chain. In 1990, Takahashi 15] pushed this up to 18 sites. And in 1994, Golinelli et al. 16 have solved for the low-lying states of a 22-site spin-1 chain. The growth in chain length of the longest spin-1 chain solved is almost linear with time, roughly increasing by 2 sites in every three years. Just to remind ourselves, the Fock space dimensionality in this case increases as $3^{N}$ with chain length $\mathrm{N}$. The size of the matrix also increases similarly and the CPU and storage scales quadratically with the size of the matrix, if we are targeting only a few eigenstates. For this reason, for systems which span much larger spaces, the focus has shifted to approximate techniques.

\section{Applications to Spin Clusters}

In recent years, some of the magnetic clusters that have been studied extensively are the $\mathrm{Mn}_{12}$ [17], $\mathrm{Fe}_{8}$ [18] and $\mathrm{V}_{15}$ [19] clusters. These clusters show many interesting phenomena such as quantum resonant tunneling and quantum interference [20]. Basic to a proper understanding of these phenomena is a knowledge of the lowenergy excitation spectrum in these systems. The methods discussed under exact diagonalization schemes allow us to calculate the low-energy excitation spectrum, given a set of exchange constants. However, the exchange constants themselves are not known with any certainty. Therefore, it is all the more important to be able to carry out exact diagonalization studies of low-lying states to infer the possible sign and magnitude of the exchange constants [7].

For the $\mathrm{Mn}_{12}$ cluster, we show the geometry and the exchange parameters in Fig. 4. The crystal structure suggests that the exchange constant $J_{1}$ is largest 
and antiferromagnetic in nature [21]. Based on magnetic measurements, it has been suggested that $J_{1}$ has a magnitude of $215 \mathrm{~K}$. The magnitude and sign of the other exchange constants are based on comparisons with manganese systems in smaller clusters. It has been suggested that the exchange constant $J_{2}$ and $J_{3}$ are antiferromagnetic and have a magnitude of about $85 \mathrm{~K}$. However, for the exchange constant $J_{4}$, there is no concrete estimate, either of the sign or of the magnitude. In an earlier study, the $\mathrm{Mn}^{I I I}-\mathrm{Mn}^{I V}$ pair with the strongest antiferromagnetic exchange constant was replaced by a composite spin-1/2 object [22] and the exchange Hamiltonian of the cluster solved for three different sets of parameters. It was found that the ordering of the energy levels were very sensitive to the relative strengths of the exchange constants. In these studies, $J_{4}$ was set to zero and the low-lying excited states were computed. Besides, only states with spin $S$ up to ten could be obtained because of the replacement of the higher spin ion pairs by the composite spin-1/2 objects.

The technique described earlier, however, allows an exact computation of the lowlying states of $\mathrm{Mn}_{12}$. The results of the exact calculations are presented in Table 1. We note that none of the three sets of parameters studied using an effective Hamiltonian, gives the correct ground and excited states, when an exact calculation is performed. It appears that setting the exchange constant $J_{4}$ to zero, cannot yield an $S=10$ ground state (Table 1 , cases A, B and C). When $J_{3}$ is equal to or slightly larger than $J_{2}$ (cases A and B, Table 1), we find a singlet ground state, unlike the result of the effective Hamiltonian in which the ground state has $S=8$ and $S=0$ respectively. The ground state has spin $S=6$, when $J_{3}$ is slightly smaller than $J_{2}$ (case $\mathrm{C}$, Table 1). In all these case, the first few low-lying states are found to lie within $20 \mathrm{~K}$ of the ground state.

When we use the parameters suggested by Chudnovsky [17] (case D, Table 1), we obtain an $S=10$ ground state separated from an $S=9$ first excited state by $223 \mathrm{~K}$. This is followed by another $S=9$ excited state at $421 \mathrm{~K}$. Only when the exchange constant $J_{4}$ is sufficiently strongly ferromagnetic (case E, Table 1), do we find an $S=10$ ground state with an $S=9$ excited state separated from it by a gap of $35 \mathrm{~K}$, which is close to the experimental value [23]. The second higher excited state has $S=8$, and is separated from the ground state by $62 \mathrm{~K}$.

In Fig. 5, we show the spin density [24] for the $\mathrm{Mn}_{12}$ cluster in the ground state for the $S=10, M_{S}=10$ state. While the manganese ions connected by the strong antiferromagnetic exchange show opposite spin densities, it is worth noting that the total spin density on these two ions is 0.69 , well away from a value of 0.5 expected if these ions were indeed to form a spin-1/2 object.

The $\mathrm{Fe}_{8}$ cluster is shown in Fig. 6. Each of the Fe ions has a spin of 2 and 
the ground state of the system has a total spin $S=10$, with $S=9$ excited state separated from it by about $20 \mathrm{~K}$. All the exchange interactions in this system are expected to be antiferromagnetic. While the structure of the complex dictates that the exchange interaction $J_{2}$ along the back of the butterfly should be small in comparison with the interaction $J_{1}$ across the wing [25], in earlier studies it was reported that such a choice of interaction parameters would not provide a $S=10$ ground state [26].

Results from the exact calculations of the eigenstates of the $\mathrm{Fe}_{8}$ cluster using three sets of parameters is shown in Table 2 . In two of these cases, $J_{2}$ is very much smaller than $J_{1}$. We find that in all these cases, the ground state has a spin $S=10$ and the lowest excited state has spin, $S=9$. One of the main difference we find amongst the three sets of parameters is in the energy gap to the lowest excited state (Table 2). For the set of parameters used in the earlier study, this gap is the lowest at $3.4 \mathrm{~K}$. For the parameter sets 1 and 3 [27, this gap is respectively $13.1 \mathrm{~K}$ and $39.6 \mathrm{~K}$. While in cases 1 and 2, the second excited state is an $S=8$ state, in case 3 , this state also has spin 9.

The spin densities in all the three cases for the ground state are shown in Fig. 7. The spin densities in all cases are positive at the corners. In cases 1 and 2, the spin density on the Fe ion on the backbone is positive and negative on the remaining two Fe sites [28]. However, in case 3, the negative and positive spin density sites for $\mathrm{Fe}$ ions in the middle of the edges is interchanged. This is perhaps due to the fact that in cases 1 and 2 , the exchange constant $J_{3}$ is less than $J_{4}$, while in case 3 ,

this is reversed. Thus, a spin density measurement can provide relative strengths of these two exchange constants. In all the three case, the difference between the spin densities in the ground and excited states is that the decrease in the spin density in the excited state is mainly confined to the corner Fe sites.

\section{Field Theoretic Studies of Spin Chains}

One-dimensional and quasi-one-dimensional quantum spin systems have been studied extensively in recent years for several reasons. Many such systems have been realized experimentally, and a variety of theoretical techniques, both analytical and numerical, are available to study the relevant models. Due to large quantum fluctuations in low dimensions, such systems often have unusual properties such as a gap between the ground state and the excited states. The most famous example of this is the Haldane gap which was predicted theoretically in integer spin Heisenberg antiferromagnetic chains [29], and then observed experimentally in a spin-1 
system $\mathrm{Ni}\left(\mathrm{C}_{2} \mathrm{H}_{8} \mathrm{~N}_{2}\right)_{2} \mathrm{NO}_{2}\left(\mathrm{ClO}_{4}\right)$ [30]. Other examples include the spin ladder systems in which a small number of one-dimensional spin- $1 / 2$ chains interact amongst each other [31]. It has been observed that if the number of chains is even, i.e., if each rung of the ladder (which is the unit cell for the system) contains an even number of spin-1/2 sites, then the system effectively behaves like an integer spin chain with a gap in the low-energy spectrum. Some two-chain ladders which show a gap are $(\mathrm{VO})_{2} \mathrm{P}_{2} \mathrm{O}_{7}$ [32, $\mathrm{SrCu}_{2} \mathrm{O}_{3}$ [33] and $\mathrm{Cu}_{2}\left(\mathrm{C}_{5} \mathrm{H}_{12} \mathrm{~N}_{2}\right)_{2} \mathrm{Cl}_{4}$ [34]. Conversely, a three-chain ladder which effectively behaves like a half-odd-integer spin chain and does not exhibit a gap is $\mathrm{Sr}_{2} \mathrm{Cu}_{3} \mathrm{O}_{5}$ [33]. A related observation is that the quasi-onedimensional system $\mathrm{CuGeO}_{3}$ spontaneously dimerizes below a spin-Peierls transition temperature [35]; then the unit cell contains two spin-1/2 sites and the system is gapped.

The results for gaps quoted above are all in the absence of an external magnetic field. The situation becomes more interesting in the presence of a magnetic field [36]. Then it is possible for an integer spin chain to be gapless and a half-odd-integer spin chain to show a gap above the ground state for appropriate values of the field [3745]. This has been demonstrated in several models using a variety of methods such as exact diagonalization of small systems and bosonization [46, 47]. In particular, it has been shown that the magnetization of the system can exhibit plateaus at certain nonzero values for some finite ranges of the magnetic field. Further, for a Hamiltonian which is invariant under translation by one unit cell, the value of the magnetization per unit cell is quantized to be a rational number at each plateau [37]. In section 8 , we will study the magnetization plateau which can occur in a three-chain ladder.

In the next two subsections, we will discuss some field theoretic methods which can be used for studying spin chains and ladders. These methods rely on the idea that the low-energy and long-wavelength modes of a system (i.e., wavelengths much

longer than the lattice spacing $a$ if the system is defined on a lattice at the microscopic level) can often be described by a continuum field theory.

\subsection{Nonlinear $\sigma$-model}

The nonlinear $\sigma$-model (NLSM) analysis of antiferromagnetic spin chains with the inclusion of $J_{2}$ (next-nearest neighbor coupling) and $\delta$ (dimerization) proceeds as follows 48]. The Hamiltonian for the frustrated and dimerized spin chain can be written as

$$
\hat{H}=\sum_{i}\left[1-(-1)^{i} \delta\right] \hat{\mathbf{S}}_{i} \cdot \hat{\mathbf{S}}_{i+1}+J_{2} \sum_{i} \hat{\mathbf{S}}_{i} \cdot \hat{\mathbf{S}}_{i+2}
$$


The interactions are schematically shown in Fig. 8. The region of interest is defined by $J_{2} \geq 0$ and $0 \leq \delta \leq 1$. We first do a classical analysis in the $S \rightarrow \infty$ limit to find the ground state configuration of the spins. Let us make the general ansatz that the ground state is a coplanar configuration of the spins with the energy per spin being equal to

$$
e_{0}=S^{2}\left[\frac{J_{1}}{2}(1+\delta) \cos \theta_{1}+\frac{J_{1}}{2}(1-\delta) \cos \theta_{2}+J_{2} \cos \left(\theta_{1}+\theta_{2}\right)\right],
$$

where $\theta_{1}$ is the angle between the spins $\mathbf{S}_{2 i}$ and $\mathbf{S}_{2 i+1}$ and $\theta_{2}$ is the angle between the spins $\mathbf{S}_{2 i}$ and $\mathbf{S}_{2 i-1}$. Minimization of the classical energy with respect to the $\theta_{i}$ yields the following three phases.

(i) Neel phase: This phase has $\theta_{1}=\theta_{2}=\pi$; hence all the spins point along the same line and they go as $\cdots \uparrow \downarrow \uparrow \downarrow \cdots$ along the chain. This phase is stable for $1-\delta^{2}>4 J_{2} / J_{1}$.

(ii) Spiral phase: Here, the angles $\theta_{1}$ and $\theta_{2}$ are given by

$$
\begin{aligned}
\cos \theta_{1} & =-\frac{1}{1+\delta}\left[\frac{1-\delta^{2}}{4 J_{2} / J_{1}}+\frac{\delta}{1+\delta^{2}} \frac{4 J_{2}}{J_{1}}\right] \\
\text { and } \cos \theta_{2} & =-\frac{1}{1-\delta}\left[\frac{1-\delta^{2}}{4 J_{2} / J_{1}}-\frac{\delta}{1-\delta^{2}} \frac{4 J_{2}}{J_{1}}\right],
\end{aligned}
$$

where $\pi / 2<\theta_{1}<\pi$ and $0<\theta_{2}<\theta_{1}$. Thus the spins lie on a plane. This phase is stable for $1-\delta^{2}<4 J_{2} / J_{1}<\left(1-\delta^{2}\right) / \delta$.

(iii) Colinear phase: This phase (which needs both dimerization and frustration) is defined to have $\theta_{1}=\pi$ and $\theta_{2}=0$; hence all the spins again point along the same line and they go as $\cdots \uparrow \uparrow \downarrow \downarrow \cdots$ along the chain. It is stable for $\left(1-\delta^{2}\right) / \delta<4 J_{2} / J_{1}$.

These phases along with their boundaries are depicted in Fig. 9. Thus even in the classical limit $S \rightarrow \infty$, the system has a rich ground state 'phase diagram' 49.

We can now go to the next order in $1 / S$, and study the spin wave spectrum about the ground state in each of the phases. The main results are as follows. In the Neel phase, we find two zero modes, i.e., modes for which the energy $\omega_{k}$ vanishes linearly at certain values of the momentum $k$, with the slope $d \omega_{k} / d k$ at those points (the velocity) being the same for the two modes. In the spiral phase, we have three zero modes, two with the same velocity describing out-of-plane fluctuations, and one with a higher velocity describing in-plane fluctuations. In the colinear phase, we get two zero modes with equal velocities just as in the Neel phase. The three phases also differ in the behavior of the spin-spin correlation function $S(q)=$ $\sum_{n}\left\langle\hat{\mathbf{S}}_{0} \cdot \hat{\mathbf{S}}_{n}\right\rangle \exp (-i q n)$ in the classical limit. $S(q)$ is peaked at $q=\left(\theta_{1}+\theta_{2}\right) / 2$, i.e., 
at $q=\pi$ in the Neel phase, at $\pi / 2<q<\pi$ in the spiral phase and at $q=\pi / 2$ in the colinear phase.

To study the interactions between the spin waves, it is convenient to derive a semiclassical NLSM field theory which can describe the low-energy and longwavelength excitations. The field theory in the Neel phase is given by a $O(3)$ NLSM with a topological term [29, 47]. The field variable is a unit vector $\vec{\phi}$ with the Lagrangian density

$$
\mathcal{L}=\frac{1}{2 c g^{2}} \dot{\vec{\phi}}^{2}-\frac{c}{2 g^{2}} \vec{\phi}^{2}+\frac{\theta}{4 \pi} \vec{\phi} \cdot \vec{\phi}^{\prime} \times \dot{\vec{\phi}}
$$

where $c=2 S\left(1-4 J_{2}-\delta^{2}\right)^{1 / 2}$ is the spin wave velocity, $g^{2}=2 /\left[S\left(1-4 J_{2}-\delta^{2}\right)^{1 / 2}\right]$ is the coupling constant (which describes the strength of the interactions between the spin waves), and $\theta=2 \pi S(1-\delta)$ is the coefficient of the topological term (the integral of this term is an integer which defines the winding number of a field configuration $\vec{\phi}(x, t))$. Note that $\theta$ is independent of $J_{2}$ in the NLSM. (Time and space derivatives are denoted by a dot and a prime respectively). For $(\theta, \bmod 2 \pi)=\pi$ and $g^{2}$ less than a critical value, it is known that the system is gapless [47, 50]. For any other value of $\theta$, the system is gapped. For $J_{2}=\delta=0$, one therefore expects that integer spin chains should have a gap while half-odd-integer spin chains should be gapless. This is known to be true even for small values of $S$ like $1 / 2$ (analytically) and 1 (numerically) although the field theory is only derived for large $S$. In the presence of dimerization, one expects a gapless system at certain special values of $\delta$. For $S=1$, the special value is predicted to be $\delta_{c}=0.5$. We see that the existence of a gapless point is correctly predicted by the NLSM. However, as we will see later, according to reliable numerical results from DMRG, $\delta_{c}$ is 0.25 for $J_{2}=0$ 51 and it decreases with $J_{2}$ as shown in Fig. 10. These deviations from field theory are probably due to higher order corrections in $1 / S$ which have not been studied analytically so far.

In the spiral phase, it is necessary to use a different NLSM which is known for $\delta=0$ [52, 53]. The field variable is now an $S O(3)$ matrix $\mathbf{R}$. The Lagrangian density is

$$
\mathcal{L}=\frac{1}{2 c g^{2}} \operatorname{Tr}\left(\dot{\mathbf{R}}^{T} \dot{\mathbf{R}} \mathbf{P}_{0}\right)-\frac{c}{2 g^{2}} \operatorname{Tr}\left(\mathbf{R}^{\prime T} \mathbf{R}^{\prime} \mathbf{P}_{1}\right)
$$

where $c=S(1+y) \sqrt{1-y^{2}} / y, g^{2}=2 \sqrt{(1+y) /(1-y)} / S$ with $1 / y=4 J_{2}$, and $\mathbf{P}_{0}$ and $\mathbf{P}_{1}$ are diagonal matrices with diagonal elements $\left(1,1,2 y(1-y) /\left(2 y^{2}-2 y+1\right)\right)$ and $(1,1,0)$ respectively. Note that there is no topological term. Hence there is no apparent difference between integer and half-odd-integer spin chains in the spiral phase. A one-loop renormalization group [52] and large $N$ analysis [53] indicate that 
the system should have a gap for all values of $J_{2}$ and $S$, and that there is no reason for a particularly small gap at any special value of $J_{2}$.

Finally, in the colinear phase, the NLSM is known for $\delta=1$, i.e., for the spin ladder. The Lagrangian is the same as in (7), with $c=4 S \sqrt{J_{2}\left(J_{2}+1\right)}, g^{2}=$ $\sqrt{1+1 / J_{2}} / S$ and $\theta=0$. There is no topological term for any value of $S$, and the model is therefore gapped.

The field theories for general $\delta$ in both the spiral and colinear phases are still not known. Although the results are qualitatively expected to be similar to the $\delta=0$ case in the spiral phase and the $\delta=1$ case in the colinear phase, quantitative features such as the dependence of the gap on the coupling strengths will require the explicit form of the field theory.

\subsection{Bosonization}

Another field theoretic method for studying spin systems in one dimension is the technique of bosonization [46, 47, 54, 55, 56]. This technique consists of mapping bosonic operators into fermionic ones, and then using whichever set of operators is easier to compute with. For instance, consider a model with a single species of fermion with a linear dispersion relation $E(k)= \pm v k$, where the \pm denotes the right- and left-moving fermions respectively (with the corresponding fields being denoted by $\hat{\psi}_{R}$ and $\hat{\psi}_{L}$ ), and $v$ denotes the velocity. Similarly, consider a model with a single species of boson with the dispersion relation $E(k)=v|k|$; the rightand left-moving fields are denoted by $\hat{\phi}_{R}$ and $\hat{\phi}_{L}$ respectively. Then it can be shown that these operators are related to each other as

$$
\begin{aligned}
\hat{\psi}_{R} & \sim \frac{1}{\sqrt{2 \pi \alpha}} e^{-i 2 \sqrt{\pi} \hat{\phi}_{R}}, \\
\hat{\psi}_{L} & \sim \frac{1}{\sqrt{2 \pi \alpha}} e^{i 2 \sqrt{\pi} \hat{\phi}_{L}} .
\end{aligned}
$$

The length parameter $\alpha$ is a cut-off which is required to ensure that the contribution from high-momentum modes do not produce divergences when computing correlation functions. It is convenient to define the two bosonic fields

$$
\begin{aligned}
& \hat{\phi}=\hat{\phi}_{R}+\hat{\phi}_{L}, \\
& \hat{\theta}=-\hat{\phi}_{R}+\hat{\phi}_{L} .
\end{aligned}
$$

Then the fermionic density is given by

$$
\hat{\rho}-\rho_{0}=\hat{\psi}_{R}^{\dagger} \hat{\psi}_{R}+\hat{\psi}_{L}^{\dagger} \hat{\psi}_{L}=-\frac{1}{\sqrt{\pi}} \frac{\partial \hat{\phi}}{\partial x},
$$


where $\rho_{0}$ is the background density; fluctuations around this density are described by the quantum fields $\hat{\psi}$ or $\hat{\phi}$.

Although the dispersion relation is generally not linear for all the modes of a given system, it often happens that the low-energy and long-wavelength modes can be studied using bosonization. For a fermionic system in one dimension, these modes are usually the ones lying close to the two Fermi points with momenta $\pm k_{F}$ respectively. One can define right- and left-moving fields $\hat{\psi}_{R}$ and $\hat{\psi}_{L}$ which vary slowly on the scale length $a$,

$$
\hat{\psi}(x)=\hat{\psi}_{R}(x) e^{i k_{F} x}+\hat{\psi}_{L}(x) e^{-i k_{F} x} .
$$

Quantities such as the density will generally contain terms which vary slowly as well as terms varying rapidly on the scale of $a$,

$$
\begin{aligned}
\hat{\rho}-\rho_{0} & =\hat{\psi}^{\dagger} \hat{\psi}=\hat{\psi}_{R}^{\dagger} \hat{\psi}_{R}+\hat{\psi}_{L}^{\dagger} \hat{\psi}_{L}+e^{-i 2 k_{F} x} \hat{\psi}_{R}^{\dagger} \hat{\psi}_{L}+e^{i 2 k_{F} x} \hat{\psi}_{L}^{\dagger} \hat{\psi}_{R} \\
& =-\frac{1}{\sqrt{\pi}} \frac{\partial \hat{\phi}}{\partial x}+\frac{1}{2 \pi \alpha}\left[e^{i\left(2 \sqrt{\pi} \hat{\phi}-2 k_{F} x\right)}+e^{-i\left(2 \sqrt{\pi} \hat{\phi}-2 k_{F} x\right)}\right] .
\end{aligned}
$$

One can compute various correlation functions in the bosonic language. Consider an operator of the form

$$
\hat{O}_{m, n}=e^{i 2 \sqrt{\pi}(m \hat{\phi}+n \hat{\theta})} .
$$

We find the following result for the two-point equal-time correlation function at spatial separations which are much larger than the microscopic lattice spacing $a$,

$$
\left\langle 0\left|T \hat{O}_{m, n}(x) \hat{O}_{m^{\prime}, n^{\prime}}^{\dagger}(0)\right| 0\right\rangle \sim \delta_{m m^{\prime}} \delta_{n n^{\prime}}\left(\frac{\alpha}{x}\right)^{2\left(m^{2} K+n^{2} / K\right)},
$$

where $K$ denotes an interaction parameter which will be described below. Note that the correlation function decays as a power law. In the language of the renormalization group, the scaling dimension of $\hat{O}_{m, n}$ is given by $m^{2} K+n^{2} / K$.

We can now study a quantum spin chain using bosonization. To be specific, let us consider a spin-1/2 chain described by the anisotropic Hamiltonian

$$
\hat{H}=\sum_{i=1}^{N}\left[\frac{J}{2}\left(\hat{S}_{i}^{+} \hat{S}_{i+1}^{-}+\hat{S}_{i}^{-} \hat{S}_{i+1}^{+}\right)+J_{z} \hat{S}_{i}^{z} \hat{S}_{i+1}^{z}-h \hat{S}_{i}^{z}\right]
$$

where the interactions are only between nearest neighbor spins, and $J>0 . \hat{S}_{i}^{+}=$ $\hat{S}_{i}^{x}+i \hat{S}_{i}^{y}$ and $\hat{S}_{i}^{-}=\hat{S}_{i}^{x}-i \hat{S}_{i}^{y}$ are the spin raising and lowering operators, and $h$ denotes a magnetic field. Note that the model has a $U(1)$ invariance, namely, rotations about the $S^{z}$ axis. When $J_{z}=J$ and $h=0$, the $U(1)$ invariance is 
enhanced to an $S U(2)$ invariance, because at this point the model can be written simply as $\hat{H}=J \sum_{i} \hat{S}_{i} \cdot \hat{S}_{i+1}$. Although the model in (16) can be exactly solved using the Bethe ansatz, and one has the explicit result that the model is gapless for a certain range of values of $J_{z} / J$ and $h / J$ (see Ref. [39]), it is not easy to compute explicit correlation functions in that approach. We therefore use bosonization to study this model.

We first use the Jordan-Wigner transformation to map the spin model to a model of spinless fermions. We map an $\uparrow$ spin or a $\downarrow$ spin at any site to the presence or absence of a fermion at that site. We introduce a fermion annihilation operator $\psi_{i}$ at each site, and write the spin at the site as

$$
\begin{aligned}
\hat{S}_{i}^{z} & =\hat{\psi}_{i}^{\dagger} \hat{\psi}_{i}-1 / 2=\hat{n}_{i}-1 / 2 \\
\hat{S}_{i}^{-} & =(-1)^{i} \hat{\psi}_{i} e^{i \pi \sum_{j} \hat{n}_{j}},
\end{aligned}
$$

where the sum runs from one boundary of the chain up to the $(i-1)^{\text {th }}$ site (we assume open boundary conditions here for convenience), $n_{i}=0$ or 1 is the fermion occupation number at site $i$, and the expression for $\hat{S}_{i}^{+}$is obtained by taking the hermitian conjugate of $\hat{S}_{i}^{-}$, The string factor in the definition of $\hat{S}_{i}^{-}$is added in order to ensure the correct statistics for different sites; the fermion operators at different sites anticommute, whereas the spin operators commute.

We now find that

$$
\hat{H}=-\sum_{i}\left[\frac{J}{2}\left(\hat{\psi}_{i}^{\dagger} \hat{\psi}_{i+1}+\text { h.c. }\right)+J_{z}\left(\hat{n}_{i}-1 / 2\right)\left(\hat{n}_{i+1}-1 / 2\right)-h\left(\hat{n}_{i}-1 / 2\right)\right] .
$$

We see that the spin-flip operators $\hat{S}_{i}^{ \pm}$lead to hopping terms in the fermion Hamiltonian, whereas the $\hat{S}_{i}^{z} \hat{S}_{i+1}^{z}$ interaction term leads to an interaction between fermions on adjacent sites.

Let us first consider the noninteracting case given by $J_{z}=0$. By Fourier transforming the fermions, $\hat{\psi}_{k}=\sum_{j} \hat{\psi}_{j} e^{-i k j a} / \sqrt{N}$, where $a$ is the lattice spacing and the momentum $k$ lies in the first Brillouin zone $-\pi / a<k \leq \pi / a$, we find that the Hamiltonian is given by

$$
\hat{H}=\sum_{k} \omega_{k} \hat{\psi}_{k}^{\dagger} \hat{\psi}_{k}
$$

where

$$
\omega_{k}=-J \cos (k a)-h .
$$

The noninteracting ground state is the one in which all the single-particle states with $\omega_{k}<0$ are occupied, and all the states with $\omega_{k}>0$ are empty. If we set the magnetic field $h=0$, the magnetization per site $m \equiv \sum_{i} S_{i}^{z} / N$ will be zero in the 
ground state; equivalently, in the fermionic language, the ground state is precisely half-filled. Thus, for $m=0$, the Fermi points $\left(\omega_{k}=0\right)$ lie at $k a= \pm \pi / 2 \equiv k_{F} a$. Let us now add the magnetic field term. In the fermionic language, this is equivalent to adding a chemical potential term (which couples to $\hat{n}_{i}$ or $\hat{S}_{i}^{z}$ ). In that case, the ground state no longer has $m=0$ and the fermion model is no longer half-filled. The Fermi points are then given by $\pm k_{F}$, where

$$
k_{F} a=\pi\left(m+\frac{1}{2}\right)
$$

It turns out that this relation between $k_{F}$ (which governs the oscillations in the correlation functions as discussed below) and the magnetization $m$ continues to hold even if we turn on the interaction $J_{z}$, although the simple picture of the ground state (with states filled below some energy and empty above some energy) no longer holds in that case.

In the linearized approximation, the modes near the two Fermi points have the velocities $\partial \omega_{k} / \partial k= \pm v$, where $v$ is some function of $J, J_{z}$ and $h$. Next, we introduce the slowly varying fermionic fields $\hat{\psi}_{R}$ and $\hat{\psi}_{L}$ as indicated above; these are functions of a coordinate $x$ which must be an integer multiple of $a$. Finally, we bosonize these fields. The spin fields can be written in terms of either the fermionic or the bosonic fields. For instance, $\hat{S}^{z}$ is given by the fermion density as in Eq. (17) which then has a bosonized form given in Eq. (13). Similarly,

$$
\begin{aligned}
\hat{S}^{+}(x)=(-1)^{x / a} & {\left[e^{-i k_{F} x / a} \hat{\psi}_{R}^{\dagger}(x)+e^{i k_{F} x / a} \hat{\psi}_{L}^{\dagger}(x)\right] \times } \\
& {\left[e^{i \pi \int_{-\infty}^{x} d x^{\prime}\left(\hat{\psi}^{\dagger}\left(x^{\prime}\right) \hat{\psi}\left(x^{\prime}\right)+1 / 2 a\right)}+\text { h.c. }\right], }
\end{aligned}
$$

where $(-1)^{x / a}= \pm 1$ since $x / a$ is an integer. This can now be written entirely in the bosonic language; the term in the exponential is given by

$$
\int_{-\infty}^{x} d x^{\prime} \hat{\psi}^{\dagger}\left(x^{\prime}\right) \hat{\psi}\left(x^{\prime}\right)=-\frac{1}{\sqrt{\pi}} \int_{-\infty}^{x} d x^{\prime} \frac{\partial \hat{\phi}}{\partial x^{\prime}}=-\frac{1}{\sqrt{\pi}}\left[\hat{\phi}_{R}(x)+\hat{\phi}_{L}(x)\right]
$$

where we have ignored the contribution from the lower limit at $x^{\prime}=-\infty$.

We can now use these bosonic expressions to compute the two-spin, equal-time correlation functions $G^{a b}(x) \equiv<0\left|T \hat{S}^{a}(x) \hat{S}^{b}(0)\right| 0>$. We find that

$$
\begin{aligned}
G^{z z}(x) & =m^{2}+\frac{c_{1}}{x^{2}}+c_{2} \frac{\cos \left(2 k_{F} x\right)}{x^{2 K}} \\
G^{+-}(x)+G^{-+}(x) & =c_{3} \frac{(-1)^{x / a}}{x^{1 / 2 K}}+c_{4} \frac{(-1)^{x / a} \cos \left(2 k_{F} x\right)}{x^{2 K+1 / 2 K}}
\end{aligned}
$$


where $c_{1}, \ldots, c_{4}$ are some constants. The parameters $K$ and $v$ are functions of $J_{z} / J$ and $h / J$; the exact dependence can be found from the web site given in Ref. [39. For $h=0, K$ is given by the analytical expression

$$
\frac{1}{K}=1+\frac{2}{\pi} \sin ^{-1}\left(\frac{J_{z}}{J}\right)
$$

Note that at the $S U(2)$ invariant point $J_{z}=J$ and $h=0$, we have $K=1 / 2$, and the two correlations $G^{z z}$ and $G^{+-}$have the same forms.

In addition to providing a convenient way of computing correlation functions, bosonization also allows us to study the effects of small perturbations. For instance, a physically important perturbation is a dimerizing term

$$
V=\delta \sum_{i}(-1)^{i}\left[\frac{J}{2}\left(\hat{S}_{i}^{+} \hat{S}_{i+1}^{-}+\hat{S}_{i}^{-} \hat{S}_{i+1}^{+}\right)+J_{z} \hat{S}_{i}^{z} \hat{S}_{i+1}^{z}\right],
$$

where $\delta$ is the strength of the perturbation. Upon bosonizing, we find that the scaling dimension of this term is $K$. Hence it is relevant if $K<2$; in that case, it produces an energy gap in the system which scales with $\delta$ as

$$
\Delta E \sim \delta^{1 /(2-K)} .
$$

This kind of phenomenon occurs in spin-Peierls systems such as $\mathrm{CuGeO}_{3}$; below a transition temperature $T_{s p}$, they go into a dimerized phase which has a gap [57].

\section{Density Matrix Renormalization Group Method}

The method which held the promise of overcoming the difficulty of exploding dimensionalities is the renormalization group $(\mathrm{RG})$ technique, in which one systematically throws out the degrees of freedom of a many-body system. While this technique found dramatic success in the Kondo problem [58], its straightforward extension to interacting lattice models was quite inaccurate [59].

In early 1992, the key problems associated with the failure of the old RG method were identified and a different renormalization procedure based on the eigenvalues of the many-body density matrix of proper subsystems was developed [60, 61]. This method has come to be known as the density matrix renormalization group (DMRG) method and has found dramatic success in solving quasi-one-dimensional many-body Hamiltonians.

In a real-space $\mathrm{RG}$ approach, one begins by subdividing the total system into several blocks $A_{n}$ and proceeds to iteratively build effective blocks so that at each 
iteration, each effective block represents two or more blocks of the previous iteration, without increasing the Fock space dimensionality of the blocks from what existed at the previous iteration. Usually, one starts with each $A_{n}$ consisting of a single site. Since the Hilbert space grows exponentially with the increase in system size, one truncates the number of states kept at each iteration.

The main reason for the failure of the old RG methods is the choice of the states retained at each stage of the iteration [60]. White [61], recognized that the weakness of the old RG procedure was in the truncation of the Fock space of a block based on the eigenvalues of the block Hamiltonian being renormalized. He replaced this choice by introducing a completely different truncation scheme than the one that was used in the old quantum RG procedures. The choice is the eigenvalues of the reduced density matrix of the block constructed from the desired state of the full Hamiltonian. The truncated Fock space is now spanned by the $m$ eigenvectors of the reduced density matrix of order $l \times l(m \leq l)$ corresponding to the $m$ highest eigenvalues of the reduced many-body density matrix. The reason for choosing the eigenvalues of the reduced density matrix as a criterion for implementing a cutoff is that, the larger the density matrix eigenvalue, the larger is the weight of the eigenstate of the density matrix in the expectation value of any property of the system. This result becomes evident when all the dynamical operators are expressed as matrices in the basis of the eigenvectors of the density matrix. The expectation value of any operator $\hat{A}$ is simply

$$
<\hat{A}>=\sum_{i} A_{i i} \rho_{i} / \sum_{i} \rho_{i},
$$

where $\rho_{i}$ is the density matrix eigenvalue. The larger the value of a particular $\rho_{i}$, the larger is its contribution to the expectation value, for a physically reasonable spread in the diagonal matrix elements $A_{i i}$.

The many-body density matrix of a part of the system can be easily constructed as follows. Let us begin with given state $\mid \psi>_{S}$ of $S$, which is called the universe or superblock, consisting of the system (which we call a block) $A$ and its environment $A^{\prime}$. Let us assume that the Fock space of $A$ and $A^{\prime}$ are known, and can be labeled as $\mid i>_{A}$ and $\mid j>_{A^{\prime}}$ respectively. The representation of $\mid \psi>_{S}$ in the product basis of $i_{A}$ and $i_{A^{\prime}}$ can be written as

$$
\left|\psi>_{S}=\sum_{i j} \psi_{i j}\right| i>_{A} \times \mid j>_{A^{\prime}}
$$

where we assume the coefficients $\psi_{i j}$ to be real, without loss of generality. Then the reduced many-body density matrix for block $A$, is defined as

$$
\rho_{k l}=\sum_{j} \psi_{k j} \psi_{l j}
$$


The eigenvalue $\rho_{i}$ of the density matrix $\rho$ gives the probability of finding the corresponding eigenstate $\mid \mu_{i}>_{A}$ in the projection of $\mid \psi>_{S}$ on block $A$. It therefore follows that the eigenvectors with highest eigenvalues of the density matrix of $A$, are the optimal or most probable states to be retained while the system is augmented.

In the early literature in quantum chemistry, the eigenvectors corresponding to large eigenvalues of one-particle density matrices were employed as the orbital basis for carrying out a configuration interaction (CI) calculation. The eigenvectors of the density matrix were called the 'natural' orbitals, and it was observed that the CI procedure converged rapidly when the 'natural' orbitals were employed in setting up the Slater determinants [62].

The DMRG scheme differs from the 'natural' orbital scheme in two important respects: (i) the reduced density matrices are many-body density matrices, and (ii) the size of the system in terms of the number of sites being studied at each iteration is usually augmented by two sites. However, the Hamiltonian matrix that one encounters from iteration to iteration, remains roughly of the same order while the matrix elements keep changing (renormalized). In this sense, the procedure can be called a renormalization procedure. The coupling constants (the Hamiltonian matrix elements) keep changing while the system size increases, as in the RG procedure carried out within a blocking technique.

\subsection{Implementation of the DMRG method}

We now describe the procedure to carry out the computations. One starts the computation with a small size system, $2 n$, which can be exactly solved, $1 \leq n \leq 4$, depending on the degree of freedom at each site. By exact diagonalization, one gets the desired eigenstate of that system. The density matrices of the left and right blocks, each consisting of $n$ sites (in principle it is not necessary to have the same number of sites for the two blocks, although in practice this is what is most generally used), are obtained from the desired eigenstate. The density matrices are diagonalized and at the first iteration usually all the density matrix eigenvectors (DMEV) are retained. The Hamiltonian matrix of the left and right blocks (denoted by $A$ and $A^{\prime}$ ) obtained in any convenient basis are transformed into the density matrix eigenvector basis. So also are the matrices corresponding to the relevant site operators in both blocks. Now, the iterative procedure proceeds as follows.

1. Construct a superblock $S=A \bullet \bullet A^{\prime}$, consisting of the block $A$, two additional sites $\sigma$ and $\sigma^{\prime}$ and the block $A^{\prime}$. Thus, at the first iteration, the system $S$ has $n+1+1+n=2 n+2$ sites. 
2. Set up the matrices for the total Hamiltonian of the superblock $S$ in the direct product basis of the DMEV of the blocks $A$ and $A^{\prime}$ and the Fock space states of the new sites. Considering that the new sites are spin- $S$ sites with $(2 S+1)$ Fock states each, the order of the total Hamiltonian matrix will be $m^{2}(2 S+1)^{2} \times m^{2}(2 S+1)^{2}$, where $m$ is the dimension of the block DMEV basis.

3. Diagonalize the Hamiltonian of the superblock $S=2 n+2$ to find the desired eigenstate $|\psi\rangle$. Using the state $|\psi\rangle$, evaluate all properties of the superblock of interest.

4. Construct the reduced many-body density matrix, $\rho$, for the new block $A \bullet$. If the system does not possess reflection symmetry, construct the density matrix, $\rho^{\prime}$, for the new right block $\bullet A^{\prime}$ as well.

5. Diagonalize the density matrix, $\rho$, and if necessary $\rho^{\prime}$. Usually, the density matrix is block-diagonal in the z-component of the total spin of the block, and it becomes computationally efficient to exploit such quantum numbers. Construct a nonsquare matrix $\mathbf{O}$, with $m$ columns, each column being an eigenvector of the density matrix corresponding to one of the $m$ largest eigenvalues. The number of rows in the matrix $\mathbf{O}$ corresponds to the order of the density matrix.

6. Construct the matrices corresponding to the Hamiltonian, $H_{A \bullet}$, of the new left block $A \bullet$, and the site spin operators $\left(\hat{S}^{z}\right.$ and $\left.\hat{S}^{+}\right)$of all the necessary sites. The $\hat{S}^{-}$operators are simply the adjoints of the $\hat{S}^{+}$operators.

7. Renormalize all the matrices corresponding to the block and site operators by using the RG transformation matrix $\mathbf{O}$, e. $g ., \tilde{H}_{A \bullet}=O H_{A} O^{\dagger}$. The resulting renormalized matrices are of order $m \times m$ and the procedure amounts to a simultaneous change of basis and a truncation.

8. Replace the block $A$ by $A \bullet$. If the system does not possess reflection symmetry, replace $A^{\prime}$ by $\tilde{A}^{\prime} \bullet$.

9. Go to step 1.

Using the block-diagonal nature of the density matrix, besides reducing the requirement in CPU time, also allows one to label the DMEV by the appropriate $z$-component of the total spin of the block $\left(M_{s, A}\right)$. The Fock space of the individual sites that are added at each iteration are eigenstates of the site spin and number 
operators. This allows us to target a definite projected spin $\left(M_{s}\right)$ state of the total system.

We now briefly describe the mathematical notations that we have used so far for various states. A state of $A \bullet$ is given by the tensor product of a state of $A$ with quantum number $q$ and an index $i$, and a state $\sigma$ of the additional site. Thus,

$$
\left|q, i, \sigma>_{A \bullet}=\right| q, i>_{A} \times \mid \sigma>
$$

A state of a superblock $S=A \bullet \bullet A^{\prime}$ is given by

$$
\left|q_{A}, \mu, \sigma ; q_{A^{\prime}}, \nu, \tau>=\right| q_{A}, \mu, \sigma>_{A} \bullet \times \mid q_{A^{\prime}}, \nu, \tau>\bullet A^{\prime}
$$

The eigenstate of the Hamiltonian of the super-block can be written as

$$
\left|\psi>_{S}=\sum_{q_{A}, q_{A^{\prime}}, \mu, \nu, \sigma, \tau} \psi_{\mu, \nu}^{q_{A}, q_{A^{\prime}}, \sigma, \tau}\right| q_{A}, \mu, \sigma ; q_{A^{\prime}}, \nu, \tau>_{S}
$$

The density matrix for $A \bullet$ then will have a block structure and can be expressed as

$$
\rho_{\mu, \nu}^{q_{A}, \sigma}=\sum_{q_{A^{\prime}, \mu^{\prime}, \tau}} \psi_{\mu, \mu^{\prime}}^{q_{A}, q_{A^{\prime}}, \sigma, \tau} \psi_{\nu, \mu^{\prime}}^{q_{A}, q_{A^{\prime}}, \sigma, \tau}
$$

The above algorithm is called the infinite lattice DMRG algorithm because this procedure is best suited for the system in the thermodynamic limit, i.e., when the properties of the system are extrapolated to the infinite system size limit.

\subsection{Finite size DMRG algorithm}

If we are interested in accurate properties of the system at a required size, then it is possible to improve upon the accuracies obtainable from the infinite DMRG procedure. This involves recognizing that the reduced many-body density matrices at each iteration correspond to a different system size. For example, when we carry out the DMRG procedure to obtain the properties of a system of $2 M$ sites, at an iteration corresponding to $2 p$ sites $(n \leq p \leq M)$, the reduced density matrix we construct is that of a block of $p$ sites in a system of $2 p$ sites. However, if our interest is in the $2 M$-site system, we should employ the density matrix of the block of $p$ sites in a $2 M$-site system. It is possible to construct, iteratively, the $p$-site reduced density matrix of the $2 M$-site system. This is achieved by the so called finite-size algorithm [61. This method provides highly accurate solutions even when the states of the full Hamiltonian have inhomogeneous (symmetry breaking) properties. 
To obtain the $2 M$-site result, we should perform the infinite lattice algorithm up to $l=(M-1)$ sites first storing all operators in each iteration. Now the algorithm for finite lattices with reflection symmetry (left block = right block), proceeds as follows.

1. On reaching a system size of $2 M$ sites, obtain the density matrix of the block of $M$ sites.

2. Use the density matrix of $M$ sites on the left and that of $(M-2)$ sites on the right, add two new sites as in the infinite DMRG procedure and obtain the desired eigenstate of the $2 M$ system.

3. Now obtain the reduced density matrix of the $(M+1)$ sites from the eigenstate of the previous iteration obtained in the direct product basis of the DMEVs of the $M$-site, $(M-2)$-site density matrices, and the Fock space states of the individual sites.

4. Go back to step 2 , replacing $M$ by $(M+1)$ and $(M-2)$ by $(M-3)$ and iterate until a single site results on the right and $(2 M-3)$ sites result on the left.

5. Since the system has reflection symmetry, use the density matrix of the $(2 M-$ 3 ) sites on the right and construct the $2 M$ system as built-up from three individual sites on the left and $(2 M-3)$ sites on the right. Obtain the desired eigenstate of the $2 M$ system in this basis.

6. Now obtain the new 2-site density matrix on the left and $(2 M-4)$ site density matrix on the right. Replace the single-site on the left by two sites and $(2 M-3)$ sites on the right by $(2 M-4)$ sites in step 5 .

7. Repeat steps 5 and 6 until $(M-1)$ sites are obtained both on the left and right. The properties of the $2 M$ system obtained from the eigenstates at this stage corresponds to the first iteration of the finite-size algorithm. We can now go back to step 1 and carry through the steps to obtain properties at later iterations of the finite-size DMRG algorithm.

In systems without reflection symmetry, the DMEVs of the right and left parts are not identical even if the sizes of the reduced systems are the same. The finiteDMRG algorithm in this case involves first constructing the density matrices of the left part for sizes greater than $M$ and on reaching the density matrix of $(2 M-3)$ sites, reducing the size of the left-part and increasing that of the right, from one site to $(M-1)$. This will result in the refined density matrices of both the right and 
the left block of the total system, for block sizes of $(M-1)$. At this stage, we can compute all the properties and continue the reverse sweep until the right block is of size $(2 M-3)$ and the left block is of size 1 . The forward sweep that follows will increase the block size on the left and decrease that of the right. We would have completed the second iteration when the two block sizes are equal. The forward and reverse sweeps can be continued until we reach the desired convergence in the properties of the whole system.

\subsection{Calculation of properties in the DMRG basis}

At the end of each iteration, one can calculate the properties of the targeted state [63]. The reduced many-body density matrix computed at each iteration can be used to calculate the static expectation values of any site operator or their products. Care should be taken to use the density matrices appropriate to the iteration. The expectation value of a site property corresponding to the operator $\hat{A}_{i}$ can be written as

$$
<\hat{A}_{i}>=\operatorname{Tr}\left(\rho \mathbf{A}_{i}\right) .
$$

$\rho$ is the density matrix of the block in which the site $i$ is situated and $\mathbf{A}_{i}$ is the matrix of the renormalized site operator at site $i$. For calculating correlation functions, one can use a similar equation. The correlation function between two site operators belonging to separate blocks can be written as

$$
<\hat{A}_{i} \hat{A}_{j}>=\operatorname{Tr}\left(\rho \mathbf{A}_{i} \mathbf{A}_{j}\right) .
$$

However, the accuracy of this procedure turns out be very poor if the sites $i$ and $j$ belong to the same block [61]. The reason is that a feature implicit in the above procedure is the resolution of identity by expansion in terms of the complete basis. Unfortunately, the basis in which the site operators are represented is incomplete and such an expansion is therefore error prone. To circumvent this difficulty, it has been suggested [61] that, one obtains the matrix representation of the products of the site operators from the first occurrence of the product pair $\langle i j\rangle$ and, by renormalizing the product operator $\hat{A}_{i} \hat{A}_{j}$, at every subsequent iteration until the

end of the RG procedure. Then, the correlation function between $\hat{A}_{i}$ and $\hat{A}_{j}$ (where $i$ and $j$ belong to the same block) can be evaluated as

$$
<\hat{A}_{i} \hat{A}_{j}>=\operatorname{Tr}\left(\rho \mathbf{A}_{i} \mathbf{A}_{j}\right) .
$$

This procedure is found to be more accurate in most cases. 


\subsection{Remarks on the applications of DMRG}

The DMRG method is currently the most accurate method for large quantum lattice models in one dimension. It can be applied to interacting bosonic, fermionic or spin models as well as to models which have interactions amongst them. The overall accuracy of the DMRG method is exceptionally high for one-dimensional systems with only nearest neighbor interactions. For a spin- $1 / 2$ chain where exact Bethe ansatz ground-state energy is available, the DMRG ground state energy per site in units of the exchange constant $J$, is found to be accurate to seven decimal places with a cut-off $m=100$ [61]. The method is found to be almost as accurate for the one-dimensional Hubbard model, where again it is possible to compare the DMRG results with exact results obtained from the exact analytic Bethe ansatz solution [64.

Since higher dimensionality is equivalent to longer-range interactions within one dimension, the model also restricts the range of interactions in one dimension. It has been noted that the number of DMEVs that should be retained in a calculation on higher dimensional systems, for accuracies comparable to accuracy in one dimension, scales exponentially with dimensionality. Thus, to obtain accuracy comparable to that obtained in a chain of $L$-sites for a cut-off $m$, in a $L \times L$ square lattice, the number of DMEVs needed to be retained for the corresponding 2-dimensional lattice is $\approx m^{2}$.

Extending the range of interactions to next-nearest neighbors does not significantly deteriorate the accuracy [65]. However, inclusion of cyclic boundary conditions reduces the accuracy of the method significantly, although in one-dimension, the DMRG method still would outperform any other method for the same system size. In the DMRG procedure, the most accurate quantity computed is the total energy. In dealing with other quantities such as correlation functions, caution must be exercised in interpreting the results.

The density matrix eigenvalues sum to unity and the truncation error, which is defined as the sum of the density matrix eigenvalues corresponding to the discarded DMEV, gives a qualitative estimate as to the accuracy of the calculation as well as providing a framework for the extrapolation to the $m \rightarrow \infty$ limit. The accuracy of the results obtained in this way is unprecedented [66, 67]. The accuracy of the ground state energy per site for the spin-1 chain is limited by the precession of machine arithmetic, i.e., $e_{0}=1.401484038971(4)$. Similarly, the accuracy persists even while calculating for the Haldane gap, e.g., the gap is evaluated to be 0.41050(2).

Another aspect of the DMRG technique worth noting is that the method is best suited for targeting one eigenstate at a time. However, it is possible to obtain 
reasonable results for a set of states by using an average many-body reduced density matrix constructed as a weighted sum of the density matrices corresponding to each of the states in question. One way of constructing the average density matrix is by using a statistical weight for the chosen set of states; the averaged density matrix in this instance is given by

$$
\rho_{\beta ; k l}=\sum_{i j} \psi_{i ; k j} \psi_{i ; l j} \exp \left[-\beta \epsilon_{i}\right] / \sum_{i} \exp \left[-\beta \epsilon_{i}\right]
$$

where $\beta=1 / k_{B} T$ with $k_{B}$ and $T$ being the Boltzmann constant and temperature respectively. One can thus extend the DMRG method to finite temperatures.

Finite size algorithms have been used extensively to study the edge states and systems with impurities, where substantial improvement of the accuracy is needed to characterize the various properties of a finite system. The DMRG method has been applied to diverse problems in magnetism: study of spin chains with $s>1 / 2$ [68, chains with dimerization and/or frustration [51, 65, 69, 70], coupled spin chains 65, 71, 72, to list a few. Highly accurate studies of the structure factor and string order parameter (topological long range order) [66] as well as edge states in Haldane phase systems [73] have been performed. Dynamical properties for both spin and fermionic systems with DMRG have also been reported within the Maximum Entropy Method [74] as well as the continued fraction [75] and correction vector [76] approaches. Also, DMRG has been successfully formulated to obtain low-temperature thermodynamic properties for various spin systems [77, 78], and the solution of models of spin chains dynamically coupled to dispersionless phonons [79]. Additionally, Nishino and Okunishi have derived two reformulations of DMRG, namely, the product wave function renormalization group (PWFRG) 80], and the corner transfer matrix renormalization group (CTMRG) [81] methods. These methods offer the means of calculating dynamical correlation functions in spin chains as well as highly accurate results for the 2-dimensional Ising model at criticality.

\section{Frustrated and Dimerized Spin Chains}

It is well known that the one-dimensional XY chain can be mapped on to a onedimensional noninteracting spinless fermion model. The isotropic spin chain will then map on to a chain of interacting spinless fermions. According to Peierls' theorem, a partly filled one-dimensional band of noninteracting fermions is unstable with respect to a lattice distortion that results in an insulating ground state. It has been shown that introduction of interactions in the Peierls system leads to a stronger instability. The mapping between the Heisenberg spin chains with equal nearest 
neighbor exchange interactions (uniform spin chain) and the spinless fermion model suggests that such a spin chain is also unstable with respect to a lattice distortion leading to alternately strong and weak nearest neighbor exchange constants, i.e., a dimerized spin chain. What is of importance is the fact that such a dimerization is unconditional: no matter how strong the lattice is, the lattice dimerizes, since the exchange energy gained due to dimerization always exceeds the strain energy. This result follows from the fact that the gain in exchange energy varies as $\delta^{2} \ln \delta$ while the strain energy loss varies as $\delta^{2}$, where $\delta$ is the magnitude of dimerization that leads to the nearest neighbor exchange constants alternating as $J(1 \pm \delta)$.

In recent years, many systems which closely approximate the one-dimensional spin chain have been synthesized. What has been observed in these experimental systems is that besides the nearest neighbor antiferromagnetic exchange, there also exists a second neighbor exchange $J_{2}$ of the same sign and comparable magnitude. Such a second neighbor interaction has the effect of frustrating the spin alignment favored by the nearest neighbor interaction. Therefore, a realistic study of these systems require modeling them using both dimerization and frustration. Theoretically, spin chains with only frustration $\left(J-J_{2}\right.$ model) were studied by Majumdar and Ghosh. Interestingly, they showed that for $J_{2}=J / 2$, the ground state is doubly degenerate and is spanned by the two possible Kekule structures (Fig. 11). It is quite gratifying to note that a century after the Kekule structure for benzene was proposed, there actually exists a Hamiltonian for which Kekule structure happens to be the ground state!

While most of the discussion above is restricted to spin- $1 / 2$ chains, there has been considerable interest in the higher-spin chains following the conjecture of Haldane which predicts that for uniform spin chains, the excitation spectrum of integer spin chains is qualitatively different from that of half-odd-integer spin chains. The latter have a gapless excitation spectrum while the excitation spectrum of the former are gapped. The synthesis and study of integer spin chains have indeed confirmed this conjecture.

Notwithstanding many interesting exact analytic solutions for spin chains, there still exist a large number of situations for which such solutions have been elusive. The exact solutions are basically confined to the uniform Heisenberg model and the frustrated and dimerized model along the line $2 J_{2}+\delta=1$ in the $J_{2}-\delta$ plane, with $J=1$. Reliable numerical study of these models therefore requires developing techniques which are highly accurate so that the results of large finite systems can be scaled or extrapolated to the thermodynamic limit. As has already been discussed, the DMRG technique is ideally suited due to its high accuracy for quasi-one-dimensional systems. 
The Hamiltonian for the frustrated and dimerized spin chain is given in Eq. (四) and is schematically shown in Fig. 8. A few low-lying states in a sector with a given value of the total spin component, $M_{S}$ are obtained in representative points in the $J_{2}-\delta$ plane, using the DMRG method. The ground state is always the first (lowest energy) state in the $M_{S}=0$ sector. The accuracy of the DMRG method depends crucially on the number of eigenstates of the density matrix, $m$, which are retained. Working with $m=100$ to 120 over the entire $J_{2}-\delta$ plane gives accurate results. This can be verified by comparing the DMRG results for these $m$ values with exact numerical diagonalizations of chains with up to 16 sites for spin-1 systems 82 and 22 sites for spin- $1 / 2$ systems [83]. The chain lengths studied vary from 150 sites for $J_{2}>0$ to 200 sites for $J_{2}=0$. The DMRG results are also tracked as a function of $N$, the chain length, to verify that convergence is reached well before 150 sites in all cases. The numerical results are much better convergent for open chains than for periodic chains, a feature generic to the DMRG technique [61, 67].

The quantum phase diagrams obtained for a spin-1/2 chain is shown in Fig. 12. The system is gapless on the line $A$ running from $J_{2}=0$ to $J_{2 c}=0.241$ for $\delta=0$, and is gapped everywhere else in the $J_{2}-\delta$ plane. There is a disorder line $B$ given by $2 J_{2}+\delta=1$; the peak in the structure factor $S(q)$ is at $q_{\max }=\pi$ to the left of $B$ (region I), decreases from $\pi$ to $\pi / 2$ as we go from $B$ up to the line $C$ (region II), and is at $q_{\max }=\pi / 2$ to the right of $C$ (region III). This is in agreement with the results obtained in section 4 using the NLSM approach. The correlation length $\xi$ goes through a minimum on line $B$.

In the spin-1 case (Fig. 10), the phase diagram is more complex. There is a solid line marked $A$ which runs from $(0,0.25)$ to about $(0.22 \pm 0.02,0.20 \pm 0.02)$ shown by a cross. To within numerical accuracy, the gap is zero on this line and the correlation length $\xi$ is as large as the system size $N$. The rest of the 'phase' diagram is gapped. However the gapped portion can be divided into different regions characterized by other interesting features. On the dotted lines marked $B$, the gap is finite. Although $\xi$ goes through a maximum when we cross $B$ in going from region II to region I or from region III to region IV, its value is much smaller than $N$. There is a dashed line $C$ extending from $(0.65,0.05)$ to about $(0.73,0)$ on which the gap appears to be zero (to numerical accuracy), and $\xi$ is very large but not as large as $N$. The straight line $D$ satisfying $2 J_{2}+\delta=1$ extends from $(0,1)$ to about $(0.432,0.136)$. Regions II and III are separated by line $E$ which goes down to about $(0.39,0)$. Across $D$ and $E$, the peak in the structure factor decreases from $\pi$ (Neel) in regions I and II to less than $\pi$ (spiral) in regions III and IV. In regions II and III, the ground state for an open chain has a four-fold degeneracy (consisting of states with $S=0$ and $S=1$ ), whereas it is nondegenerate in regions I and IV with $S=0$. The regions II and III, 
where the ground state is four-fold degenerate for an open chain, can be identified with the Haldane phase; the regions I and IV correspond to a non-Haldane singlet phase. The lines $B, D$ and $E$ meet in a small region $\mathrm{V}$ where the ground state of the system is numerically very difficult to find. Note that the numerically zero gap at $(0.73,0)$ is unexpected from either bosonic mean-field theory 84 or the NLSM approach discussed earlier.

For the spin-1 system, there is a striking similarity between the ground state properties of the dimerized and frustrated model (何) as a function of $J_{2}$ (with $\delta=0$ ) and the biquadratic model,

$$
\hat{H}=\sum_{i}\left[\hat{\mathbf{S}}_{i} \cdot \hat{\mathbf{S}}_{i+1}+\beta\left(\hat{\mathbf{S}}_{i} \cdot \hat{\mathbf{S}}_{i+1}\right)^{2}\right],
$$

as a function of (positive) $\beta$ [69]. For $J_{2}<0.39$ and $\beta<1 / 3$, both models are in the Neel phase and are gapped. For $J_{2}>0.39$ and $\beta>1 / 3$, the two models are in the spiral phase and are generally gapped; however, model (44) is 'gapless' for $J_{2}=0.73$ while model (39) is gapless for $\beta=1$. Qualitatively, the cross-over from the Neel to the spiral phase (but not the gaplessness at a particular value of $J_{2}$ or $\beta$ ) can be understood through the following classical argument. Let us set the magnitudes of the spins equal to 1 and define the angle between spins $\mathbf{S}_{i}$ and $\mathbf{S}_{i+n}$ to be $n \theta$. The angle $\theta$ can be obtained by minimizing $\cos \theta+J_{2} \cos 2 \theta$ in (4), and $\cos \theta+\beta \cos ^{2} \theta$ in (39). This gives us a Neel phase $(\theta=\pi)$ if $J_{2} \leq 1 / 4$ and $\beta \leq 1 / 2$ in the two models, and a spiral phase for larger values of $J_{2}$ and $\beta$ with $\theta=\cos ^{-1}\left(-1 / 4 J_{2}\right)$ and $\theta=\cos ^{-1}(-1 / 2 \beta)$ respectively. The actual crossover points from Neel to spiral is different for spin-1 than these classical values. In the classical limit $S \rightarrow \infty$, the ground state of the model is in the Neel phase for $4 J_{2}<1-\delta^{2}$, in a spiral phase for $1-\delta^{2}<4 J_{2}<\left(1-\delta^{2}\right) / \delta$ and in the colinear phase for $\left(1-\delta^{2}\right) / \delta<4 J_{2}$ (Fig. 9).

As can be seen from Fig. $8, \delta=1$ results in two coupled spin chains wherein the interchain coupling is 2 and the intrachain coupling is $J_{2}$. By using DMRG, one can study the dependence of the gap $\Delta$ and the two-spin correlation function $C(r)$ on the interchain coupling $J$. In Fig. 13 is plotted $\Delta$ vs $J$ for both spin-1/2 and spin- 1 systems. For spin-1/2, the system is gapped for any nonzero value of the interchain coupling $J$, although the gap vanishes as $J \rightarrow 0$. The gap increases and correspondingly the correlation length decreases with increasing $J$. In the case of coupled spin-1 chains, one finds the somewhat surprising result that both the gap and the correlation length $\xi$ are fairly large for moderate values of $J$. Note that the variation of the gap with $J$ for spin-1 (shown as circles) is much less than that for spin-1/2 (crosses).

The NLSMs derived in section 4 can be expected to be accurate only for large values of the spin $S$. It is interesting to note that the numerically obtained 'phase' 
boundary between the Neel and spiral phases for spin-1 is closer to the the classical $(S \rightarrow \infty)$ boundary $4 J_{2}=1-\delta^{2}$ than for spin-1/2. For instance, the crossover from Neel to spiral occurs, for $\delta=0$, at $J_{2}=0.5$ for spin- $1 / 2$, at 0.39 for spin- 1 , and at 0.25 classically.

To conclude this section, we have studied a two-parameter 'phase' diagram for the ground state of isotropic antiferromagnetic spin-1/2 and spin- 1 chains. The spin1 diagram is considerably more complex than the corresponding spin- $1 / 2$ chain, with surprising features like a 'gapless' point inside the spiral 'phase'; this point could be close to a critical point discussed earlier in the literature [50, 85]. It would be interesting to establish this more definitively. Our results show that frustrated spin chains with small values of $S$ exhibit features not anticipated from large $S$ field theories.

\section{Alternating $\left(S_{1}, S_{2}\right)$ ferrimagnetic spin chains}

Ferrimagnets belong to a class of magnets which show spontaneous magnetization below a certain critical temperature. There have been a number of experimental efforts to synthesize molecular materials showing spontaneous magnetization at low temperatures [86, 87]. These are quasi-one-dimensional bimetallic molecular magnets in which each unit cell contains two spins with different spin values, with the general formula $\mathrm{ACu}(\mathrm{pbaOH})\left(\mathrm{H}_{2} \mathrm{O}\right)_{3} \cdot 2 \mathrm{H}_{2} \mathrm{O}$, where pbaOH is 2-hydroxo1,3-propylenebis(oxamato) and $\mathrm{A}=\mathrm{Mn}, \mathrm{Fe}, \mathrm{Co}, \mathrm{Ni}$; they belong to the alternating or mixed spin chain family 87, 88. These alternating spin compounds have been seen to exhibit ferrimagnetic behavior. It has been possible to vary the spin at each site from low values where quantum effects dominate to large values which are almost classical.

The thermodynamic behavior of these alternating spin compounds is very interesting [88, 89]. In very low magnetic fields, these systems show one-dimensional ferrimagnetic behavior. The $\chi T$ vs $T$ (where $\chi$ is the magnetic susceptibility and $T$ the temperature) plots show a rounded minimum. As the temperature is increased, $\chi T$ decreases sharply, goes through a minimum before increasing gradually. The temperature at which this minimum occurs differs from system to system and depends on the site spins of the chain. The variation of the field induced magnetization with temperature is also interesting as the ground state is a magnetic state. These exciting observations have motivated us to study the ferrimagnetic systems with arbitrary spins $s_{1}$ and $s_{2}$ alternating from site to site. It would also be of interest to know the thermodynamic properties of these systems with varying $s_{1}$ and $s_{2}$. 


\subsection{Ground state and excitation spectrum}

We start our discussion with the Hamiltonian for a chain with spins $s_{1}$ and $s_{2}$ on alternating sites (with $s_{1}>s_{2}$, without loss of generality).

$$
\hat{H}=J \sum_{n}\left[(1+\delta) \hat{\mathbf{S}}_{1, n} \cdot \hat{\mathbf{S}}_{2, n}+(1-\delta) \hat{\mathbf{S}}_{2, n} \cdot \hat{\mathbf{S}}_{1, n+1}\right]
$$

where the total number of sites is $2 N$ and the sum is over the total number of unit cells $N . \hat{\mathbf{S}}_{i, n}$ corresponds to the spin operator for the site spin $s_{i}$ in the $n$-th unit cell. The exchange integral $J$ is taken to be positive for all our calculations; $\delta$ is the dimerization parameter and it lies in the range $\{0,1\}$.

Before describing our numerical results, we briefly summarize the results of a spin wave analysis for the purposes of comparison [78. We will first state the results for $\delta=0$. According to spin wave theory, the ground state has total spin $S_{G}=N\left(s_{1}-s_{2}\right)$. Let us define a function

$$
\omega(k)=J \sqrt{\left(s_{1}-s_{2}\right)^{2}+4 s_{1} s_{2} \sin ^{2}(k / 2)},
$$

where $k$ denotes the wave number. Then the ground state energy per site is given by

$$
\epsilon_{0}=\frac{E_{0}}{2 N}=-J s_{1} s_{2}+\frac{1}{2} \int_{0}^{\pi} \frac{d k}{\pi}\left[-J\left(s_{1}+s_{2}\right)+\omega(k)\right] .
$$

The lowest branch of excitations is to states with spin $S=S_{G}-1$, with the dispersion

$$
\omega_{1}(k)=J\left(-s_{1}+s_{2}\right)+\omega(k) ;
$$

the gap vanishes at $k=0$. There is a gapped branch of excitations to states with spin $S=S_{G}+1$, with the dispersion

$$
\omega_{2}(k)=J\left(s_{1}-s_{2}\right)+\omega(k)
$$

the minimum gap occurs at $k=0$ and is given by $\Delta=2 J\left(s_{1}-s_{2}\right)$. In the ground state with $S^{z}=S_{G}$, the sublattice magnetizations are given by the expectation values

$$
\begin{aligned}
& <\hat{S}_{1, n}^{z}>=\left(s_{1}+\frac{1}{2}\right)-\frac{1}{2} \int_{0}^{\pi} \frac{d k}{\pi} \frac{J\left(s_{1}+s_{2}\right)}{\omega(k)} \\
& <\hat{S}_{2, n}^{z}>=s_{1}-s_{2}-<\hat{S}_{1, n}^{z}>
\end{aligned}
$$

The various two-spin correlation functions decay exponentially with distance; the inverse correlation length is given by $\xi^{-1}=\ln \left(s_{1} / s_{2}\right)$. The results with dimerization 
$(\delta>0)$ are very similar. In fact, within spin wave theory, the minimum gap $\Delta$ to states with spin $S=S_{G}+1$ is independent of $\delta$.

We now use the powerful DMRG method to study the system defined by Eq. (40) both with and without dimerization, $\delta \neq 0$ and $\delta=0$ respectively. We have considered alternating spin-3/2/spin-1 (hereafter designated as $(3 / 2,1)$ ), spin-3/2/spin-1/2 (to be called $(3 / 2,1 / 2))$ and spin-1,1/2 (called $(1,1 / 2))$ chains with open boundary condition for the Hamiltonian (40). We compute the ground state properties for these three systems by studying chains with 80 to 100 sites. The number of dominant density matrix eigenstates, $m$, that we have retained at each DMRG iteration also varies between 80 to 100 . With the increase of the Fock space dimensionality of the site spins, we increase $m$ to obtain more accurate results. We follow the usual steps for the "infinite system" DMRG method discussed above [61, 65, 90], except that the alternating chains studied here are not symmetric between the left and right halves; hence the density matrices for these two halves have to be separately constructed at every iteration of the calculations. We have also verified the convergence of our results by varying the values of $m$ and the system size.

The ground states of all the systems lie in the $S^{z}=N\left(s_{1}-s_{2}\right)$ sector, as verified from extensive checks carried out by obtaining the low-energy eigenstates in different $S^{z}$ sectors of a 20-site chain. A state corresponding to the lowest energy in $S^{z}=N\left(s_{1}-s_{2}\right)$ is found in all subspaces with $\left|S^{z}\right| \leq N\left(s_{1}-s_{2}\right)$, and is absent in subspaces with $\left|S^{z}\right|>N\left(s_{1}-s_{2}\right)$. This shows that the spin in the ground state is $S_{G}=N\left(s_{1}-s_{2}\right)$. (Actually, the lowest energy states in the different $S^{z}$ sectors are found to be degenerate only up to $10^{-5} \mathrm{~J}$. Such small errors are negligible for studying thermodynamics at temperatures larger than, say, $10^{-2} \mathrm{~J}$ ).

In Fig. 14, we show the expectation value of site spin operator $\hat{S}_{i, n}^{z}$ ( spin density) at all the sites for the $(3 / 2,1),(3 / 2,1 / 2)$ and $(1,1 / 2)$ chains. The spin densities are uniform on each of the sublattices in the chain for all the three systems. For the $(3 / 2,1)$ chain, the spin density at a spin-3/2 is 1.14427 (the classical value is $3 / 2)$, while, at a spin- 1 site it is -0.64427 (classical value 1 ). For the $(3 / 2,1 / 2)$ case, the spin density at a spin-3/2 site is 1.35742 and at a spin- $1 / 2$ site it is -0.35742 . For the $(1,1 / 2)$ case, the value at a spin- 1 site is 0.79248 and at a spin- $1 / 2$ site it is -0.29248 . These can be compared with the spin wave values of 1.040 and -0.540 ; 1.314 and -0.314 ; and 0.695 and -0.195 for the spin- $s_{1}$ and spin- $s_{2}$ sites of the $(3 / 2,1) ;(3 / 2,1 / 2)$; and $(1,1 / 2)$ systems respectively. We note that the spin wave analysis overestimates the quantum fluctuations in case of systems with small site spin values. We also notice that there is a greater quantum fluctuation when the difference in site spin $\left|s_{1}-s_{2}\right|$ is larger. This is also seen in spin-wave theory. The spin density distribution in an alternating $\left(s_{1}, s_{2}\right)$ chain is more similar to that of 
a ferromagnetic chain rather than an antiferromagnet, with the net spin of each unit cell perfectly aligned (but with small quantum fluctuations on the individual sublattices). In a ferromagnetic ground state, the spin density at each site has the classical value appropriate to the site spin, whereas for an antiferromagnet, this averages out to zero at each site as the ground state is nonmagnetic. From this viewpoint, the ferrimagnet is similar to a ferromagnet and is quite unlike an antiferromagnet. The spin wave analysis also yields the same physical picture.

Because of the alternation of spin- $s_{1}$ and spin- $s_{2}$ sites along the chain, one has to distinguish between three different types of pair correlations, namely, $<\hat{S}_{1,0}^{z} \hat{S}_{1, n}^{z}>$, $<\hat{S}_{2,0}^{z} \hat{S}_{2, n}^{z}>$ and $<\hat{S}_{1,0}^{z} \hat{S}_{2, n}^{z}>$. We calculate all the three correlation functions with the mean values subtracted out, since the mean values are nonzero in all these three systems unlike in pure antiferromagnetic spin chains. In the DMRG procedure, we have computed these correlation functions from the sites inserted at the last iteration, to minimize numerical errors. In Fig. 15, we plot the two-spin correlation functions in the ground state as a function of the distance between the spins for an open chain of 100 sites for all three cases. All three correlation functions decay rapidly with distance for each of the three systems. From the figure it is clear that, except for the $<\hat{S}_{1,0}^{z} \hat{S}_{2, n}^{z}>$ correlation, all other correlations are almost zero even for the shortest possible distances. The $<\hat{S}_{1,0}^{z} \hat{S}_{2, n}^{z}>$ correlation has an appreciable value $[-0.2$ for $(3 / 2,1),-0.07$ for $(3 / 2,1 / 2)$ and -0.094 for $(1,1 / 2)]$ only for the nearest neighbors. This rapid decay of the correlation functions makes it difficult to find the exact correlation length $\xi$ for a lattice model, although it is clear that $\xi$ is very small (less than one unit cell) for the $(3 / 2,1 / 2)$ and $(1,1 / 2)$ cases, and a little greater $(1<\xi<2)$ for the $(3 / 2,1)$ system. Spin wave theory gives $\xi=2.47$ for $(3 / 2,1), \xi=0.91$ for $(3 / 2,1 / 2)$, and $\xi=1.44$ for $(1,1 / 2)$ cases. (We should remark here that our $\xi$ is not to be confused with the conventional definition of the correlation length; the latter is actually infinite in these systems due to the long-range ferrimagnetic order).

The lowest spin excitation of all the three chains is to a state with $S=S_{G}-1$. To study this state, we target the $2^{\text {nd }}$ state in the $S^{z}=S_{G}-1$ sector of the chain. To confirm that this state is a $S=S_{G}-1$ state, we have computed the $2^{\text {nd }}$ state in $S^{z}=0$ sector and find that it also has the same energy. However, the corresponding state is absent in $S^{z}$ sectors with $\left|S^{z}\right|>S_{G}-1$. Besides, from exact diagonalization of all the states of all the $s_{1}-s_{2}$ alternating spin chains with 8 sites, we find that the energy orderings of the states is such that the lowest excitation is to a state with spin $S=S_{G}-1$. We have obtained the excitation gaps for all the three alternating spin chains in the limit of infinite chain length by extrapolating from the plot of spin gap vs the inverse of the chain length (Fig. 16). We find that this excitation is 
gapless in the infinite chain limit for all three cases.

To characterize the lowest spin excitations completely, we also have computed the energy of the $S=S_{G}+1$ state by targeting the lowest state in the $S^{z}=S_{G}+1$ sector. In Fig. 17, we plot the excitation gaps to the $S=S_{G}+1$ state from the ground state for all three systems as a function of the inverse of the chain length. The gap saturates to a finite value of $(1.0221 \pm 0.0001) J$ for the $(3 / 2,1)$ case, $(1.8558 \pm 0.0001) J$ for $(3 / 2,1 / 2)$, and $(1.2795 \pm 0.0001) J$ for $(1,1 / 2)$. It appears that the gap is also higher when the difference in site spins $\left|s_{1}-s_{2}\right|$, is larger. The site spin densities expectation values computed in this state for all three cases are found to be uniform (i.e., independent of the site) on each of the sublattices. This leads us to believe that this excitation cannot be characterized as the states of a magnon confined in a box, as has been observed for a spin-1 chain in the Haldane phase 66.

We have also studied the spin excitations in the dimerized alternating $\left(s_{1}, s_{2}\right)$ chains, defined in Eq. (40). We calculate the lowest spin excitation to the $S=S_{G}-1$ state from the ground state. We find that the $S=S_{G}-1$ state is gapless from the ground state for all values of $\delta$. This result agrees with the spin wave analysis of the general $\left(s_{1}, s_{2}\right)$ chain. The systems remain gapless even while dimerized unlike the pure antiferromagnetic dimerized spin chains. There is a smooth increase of the spin excitation gap from the ground state to the $S=S_{G}+1$ state with increasing $\delta$ for all three systems studied here. We have plotted this gap vs $\delta$ in Fig. 18. The gap shows almost a linear behavior as a function of $\delta$, with an exponent of $1.0 \pm 0.01$ for all three systems. This seems to be an interesting feature of all ferrimagnets. The spin wave analysis however shows that this excitation gap is independent of $\delta$ for the general $\left(s_{1}, s_{2}\right)$ chain. The similar behaviors of these three alternating spin systems suggest that a ferrimagnet can be considered as a ferromagnet with small quantum fluctuations.

\subsection{Low-temperature thermodynamic properties}

We have varied the size of the system from 8 to 20 sites to calculate the thermodynamic properties. We impose periodic boundary conditions to minimize finite size effects with $\hat{\mathbf{S}}_{1, N+1}=\hat{\mathbf{S}}_{1,1}$, so that the number of sites equals the number of bonds. We set up the Hamiltonian matrices in the DMRG basis for all allowed $S^{z}$ sectors for a ring of $2 N$ sites. We can diagonalize these matrices completely to obtain all the eigenvalues in each of the $S^{z}$ sectors. As the number of DMRG basis states increases rapidly with increasing $m$, we retain a smaller number of dominant density matrix eigenvectors in the DMRG procedure, i.e., $50 \leq m \leq 65$, depending on 
the $S^{z}$ sector as well as the size of the system. We have checked the dependence of properties (with $m$ in the range $50 \leq m \leq 65$ ) for the system sizes we have studied $(8 \leq 2 N \leq 20)$, and have confirmed that the properties do not vary significantly for the temperatures at which they are computed; this is true for all the three systems.

It may appear surprising that the DMRG technique which essentially targets a single state, usually the lowest energy state in a chosen $S^{z}$ sector, should provide accurate thermodynamic properties since these properties are governed by the energy level spacings and not by the absolute energy of the ground state. However, there are two reasons why the DMRG procedure yields reasonable thermodynamic properties at low temperatures. Firstly, the projection of the low-lying excited state eigenfunctions on the DMRG space which contains the ground state is substantial; hence these excited states are well described in the chosen DMRG space. Secondly, the low-lying excitations of the full system are often the lowest energy states in different sectors in the DMRG procedure; hence their energies are quite accurate even on an absolute scale.

The canonical partition function $Z$ for the $2 N$ site ring can be written as

$$
Z=\sum_{j} e^{-\beta\left(E_{j}-B(M)_{j}\right)}
$$

where the sum is over all the DMRG energy levels of the $2 N$ site system in all the $S^{z}$ sectors. $E_{j}$ and $(M)_{j}$ denote the energy and the $\hat{z}$-component of the total spin of the state $j$, and $B$ is the strength of the magnetic field in units of $1 / g \mu_{B}$ ( $g$ is the gyromagnetic ratio and $\mu_{B}$ is the Bohr magneton) along the $\hat{z}$ direction. The field induced magnetization $\langle M\rangle$ is defined as

$$
<M>=\frac{\sum_{j}(M)_{j} e^{-\beta\left(E_{j}-B(M)_{j}\right)}}{Z} .
$$

The magnetic susceptibility $\chi$ is related to the fluctuation in magnetization

$$
\chi=\beta\left[<M^{2}>-<M>^{2}\right] \text {. }
$$

Similarly, the specific heat $C_{V}$ is related to the fluctuation in the energy and can be written as

$$
\frac{C_{V}}{k_{B}}=\beta^{2}\left[<E^{2}>-<E>^{2}\right] .
$$

In the discussion to follow, we present results on the 20-site ring although all calculations have been carried out for system sizes from 8 to 20 sites. This is because the qualitative behavior of the properties we have studied are similar for all the ring sizes in this range for all three systems. 
The dependence of magnetization on temperature for different magnetic field strengths are shown in Fig. 19 for all three systems. At low magnetic fields, the magnetization shows a sharp decrease at low temperatures and shows paramagnetic behavior at high temperatures. As the field strength is increased, the magnetization shows a slower decrease with temperature, and for high field strengths the magnetization shows a broad maximum. This behavior can be understood from the type of spin excitations present in these systems. The lowest energy excitation at low magnetic fields is to a state with spin $s$ less than $S_{G}$. Therefore, the magnetization initially decreases at low temperatures. As the field strength is increased, the gap to spin states with $S>S_{G}$ decreases as the Zeeman coupling to these states is stronger than to the states with $S \leq S_{G}$. The critical field strengths at which the magnetization increases with temperature varies from system to system since this corresponds to the lowest spin gap of the corresponding system. The behavior of the system at even stronger fields turns out to be remarkable. The magnetization in the ground state $(T=0)$ shows an abrupt increase signalling that the ground state at this field strength has $S^{z}>S_{G}$. The temperature dependence of the magnetization shows a broad maximum indicating the presence of states with even higher spin values lying above the ground state in the presence of this strong field. In all three cases, the ground state at very high field strengths should be ferromagnetic. For the systems at such high fields, the magnetization decreases slowly with increase of temperature as no other higher spin states lie above the ground state. While we have not studied such high field behaviors, we find that the field strength corresponding to switching the spin of the ground state $s_{G}$ to $s_{G}+1$ is higher for $(3 / 2,1 / 2)$ system compared to $(3 / 2,1)$ and $(1,1 / 2)$ systems. The switching field appears to depend on the value of $\left|s_{1}-s_{2}\right|$. We see in Fig. 19 that in the $(3 / 2,1)$ and $(1,1 / 2)$ cases, the ground state has switched to the higher spin state at the highest magnetic field strength we have studied but in the $(3 / 2,1 / 2)$ case, the ground state has not switched even at the field strength indicating that the excitation gap for this system is larger than the other two. For the $(3 / 2,1 / 2)$ case, the same situation should occur at very high magnetic fields. Thus, we predict that the highest $S^{z}$ is attained in the ground state at high magnetic field and this field strength increases with increase in the site spin difference $\left|s_{1}-s_{2}\right|$.

The dependence of $\chi T / 2 N$ on temperature for different field strengths are shown in Fig. 20 for all three systems. For zero field, the zero temperature value of $\chi T$ is infinite in the thermodynamic limit; for finite rings it is finite and equal to the average of the square of the magnetization in the ground state. For the ferrimagnetic ground state $\chi T / 2 N$, as $T \rightarrow 0$, is given by $S_{G}\left(S_{G}+1\right) / 6 N$. As the temperature increases, this product decreases and shows a minimum before increasing again. For the three systems studied here, the minimum occurs at different temperatures depending on 
the system. For the $(3 / 2,1)$ alternating spin system, it is at $k_{B} T=(0.8 \pm 0.1) \mathrm{J}$, while for the $(3 / 2,1 / 2)$ and $(1,1 / 2)$ cases, it occurs at $k_{B} T=(1.0 \pm 0.1) J$ and $k_{B} T=(0.5 \pm 0.1) J$ respectively. The minimum occurs due to the states with $S^{z}<S_{G}$ getting populated at low temperatures. In the infinite chain limit, these states turn out to be the gapless excitations of the system. The subsequent increase in the product $\chi T$ is due to the higher energy-higher spin states being accessed with further increase in temperature. This increase is slow in $(3 / 2,1 / 2)$ case, as in this system very high spin states are not accessible within the chosen temperature range. Experimentally, it has been found in the bimetallic chain compounds that the temperature at which the minimum occurs in the $\chi T$ product depends upon the magnitude of the spins $s_{1}$ and $s_{2}$ [89]. The $N i^{I I}-C u^{I I}$ bimetallic chain shows a minimum in $\chi T / 2 N$ at a temperature corresponding to $55 \mathrm{~cm}^{-1}(80 \mathrm{~K})$; an independent estimate of the exchange constant in this system is $100 \mathrm{~cm}^{-1}$ [91]. This is in very good agreement with the minimum theoretically found at temperature $(0.5 \pm 0.1) \mathrm{J}$ for the $(1,1 / 2)$ case. The minimum in $\chi T / 2 N$ vanishes at $B=0.1 \mathrm{~J} / g \mu_{B}$ which corresponds to about $10 \mathrm{~T}$ for all three systems. It would be interesting to study the magnetic susceptibility of these systems experimentally under such high fields. The low-temperature zero-field behavior of our systems can be compared with the one-dimensional ferromagnet. In the latter, the spin wave analysis shows that the $\chi T$ product increases as $1 / T$ at low temperatures [92.

In finite but weak fields, the behavior of $\chi T$ is different. The magnetic field opens up a gap and $\chi T$ falls exponentially to zero for temperatures less than the gap in the applied field for all three systems. Even in this case a minimum is found at the same temperature as in the zero-field case for the corresponding system, for the same reason as discussed in the zero field case.

In stronger magnetic fields, the behavior of $\chi T$ from zero temperature up to $k_{B} T=J_{\min }\left(J_{\min }\right.$ is the temperature at which the minimum in $\chi T$ is observed $)$ is qualitatively different. The minimum in this case vanishes for all three systems. In these field strengths, the states with higher $S^{z}$ values are accessed even below $k_{B} T=J_{\min }$. The dependence of $\chi T$ above $k_{B} T=J_{\min }$ at all field strengths is the same in all three systems. In even stronger magnetic fields, the initial sharp increase is suppressed. At very low temperature, the product $\chi T$ is nearly zero and increases almost linearly with $T$ over the temperature range we have studied. This can be attributed to a switch in the ground state at this field strength. The very high temperature behavior of $\chi T$ should be independent of field strength and should saturate to the Curie law value corresponding to the mean of magnetic moments due to spin- $s_{1}$ and spin- $s_{2}$.

The temperature dependence of specific heat also shows a marked dependence on 
the magnetic field at strong fields. This dependence is shown in Fig. 21 for various field strengths for all the three systems. In zero and weak magnetic fields, the specific heat shows a broad maximum at different temperatures which are specific to the system. Interestingly, the temperature at which the specific heat shows a maximum closely corresponds to the temperature where the low-field $\chi T$ has a minimum for the corresponding system. For a strong magnetic field $(B \sim J)$, there is a dramatic increase in the peak height at about the same temperature corresponding to the specific system, although the qualitative dependence is still the same as at low magnetic fields in all three cases. This phenomena indicates that the higher energy high-spin states are brought to within $k_{B} T$ of the ground state at this magnetic field strength for all three cases.

Studies of thermodynamic properties of the dimerized alternating spin chains in these three cases show qualitatively similar trends to that of the corresponding uniform systems; this follows from the fact that the low-energy spectrum does not change qualitatively upon dimerization.

\section{Magnetization Properties of a Spin Ladder}

As mentioned earlier, a quantum spin system can sometimes exhibit magnetization plateaus as a function of an applied magnetic field [37-45]. In this section, we will use the finite system DMRG method to study the magnetic properties of a three-legged spin-1/2 ladder [93. We consider the Hamiltonian

$$
\hat{H}=J^{\prime} \sum_{a} \sum_{n} \hat{\mathbf{S}}_{a, n} \cdot \hat{\mathbf{S}}_{a+1, n}+J \sum_{a=1}^{3} \sum_{n} \hat{\mathbf{S}}_{a, n} \cdot \hat{\mathbf{S}}_{a, n+1}-h \sum_{a=1}^{3} \sum_{n} \hat{S}_{a, n}^{z},
$$

where $a$ denotes the chain index, $n$ denotes the rung index, $h$ denotes the magnetic field (we have absorbed the gyromagnetic ratio $g$ and the Bohr magneton $\mu_{B}$ in the definition of $h$ ), and $J, J^{\prime}>0$. It is convenient to scale out the parameter $J$, and quote all results in terms of the two dimensionless quantities $J^{\prime} / J$ and $h / J$. If the length of each chain is $L$, the total number of sites is $N=3 L$. Since the total $\hat{S}^{z}$ is a good quantum number, it is more convenient to do the numerical computations without including the magnetic-field term in (50), and then to add the effect of the field at the end of the computation. For the ground state properties, we have only considered an open boundary condition (OBC) in the rung direction, namely, the summation over $a$ in the first term of (50) runs over 1,2. However, for lowtemperature properties, we have studied both OBC, as well as a periodic boundary condition (PBC) in the rung direction in which we sum over $a=1,2,3$ in the first term. 
We have done DMRG calculations (using the finite system algorithm 61]) with open boundary conditions in the chain direction. We have gone up to 120 sites, i.e., a chain length of 40. The number of dominant density matrix eigenstates, corresponding to the $m$ largest eigenvalues of the density matrix, that we retained at each DMRG iteration was $m=80$. In fact, we varied the value of $m$ from 60 to 100 , and found that $m=80$ gives satisfactory results in terms of agreement with exact diagonalization for small systems and good numerical convergence for large systems. For inputting the values of the couplings into the numerical programmes, it is more convenient to think of the system as a single chain (rather than as three chains) with the Hamiltonian

$$
\hat{H}=\frac{2}{3} J^{\prime} \sum_{i}\left[1-\cos \left(\frac{2 \pi i}{3}\right)\right] \hat{\mathbf{S}}_{i} \cdot \hat{\mathbf{S}}_{i+1}+J \sum_{i} \hat{\mathbf{S}}_{i} \cdot \hat{\mathbf{S}}_{i+3}
$$

The system is grown by adding two new sites at each iteration. Note that our method of construction ensures that we obtain the three-chain ladder structure after every third iteration when the total number of sites becomes a multiple of 6 . At various system sizes, starting from 48 sites and going up to 120 sites in multiples of 6 sites, we computed the energies after doing three finite system iterations; we found that the energy converges very well after three iterations. The energy data is used in Figs. 22 and 23 below. After reaching 120 sites, we computed the spin correlations after doing three finite system iterations. This data is used in Figs. 24 and 25. All our numerical results quoted below are for $J / J^{\prime}=1 / 3$. We chose this particular value of the ratio because there is a particularly broad magnetization plateau at $m_{s}=1 / 2$ which can be easily found numerically.

We now describe the various ground-state properties we have found with OBC along the rungs. We looked for magnetization plateaus at $m_{s}=0,1 / 2$ and 1 . For a system with $N$ sites, a given value of magnetization per rung, $m_{s}$, corresponds to a sector with total $S^{z}$ equal to $M=m_{s} N / 3$. Using the infinite system algorithm, we found the lowest energies $E_{0}\left(S^{z}, N\right)$ in the three sectors $S^{z}=M+1, M$ and $M-1$. Then we examined the three plots of $E_{0} / N J$ vs $1 / N$ and extrapolated the results up to the thermodynamic limit $N \rightarrow \infty$. We fitted these plots with the formula $E_{0} / N J=e_{i}+a_{i} / N+b_{i} / N^{2}$, where the label $i=1,2,3$ denotes the $S^{z}$ sectors $M+1, M$ and $M-1$. In the thermodynamic limit, the values of the three intercepts $e_{i}$ should match since those are just the energy per site for the three states whose $S^{z}$ 's differ by only 1 . However, the three slopes $a_{i}$ are not equal in general. We now show that there is a magnetization plateau if $a_{1}+a_{3}-2 a_{2}$ has a nonzero value. Since the three energies $E_{0}$ are computed without including the magnetic field term, the upper critical field $h_{c+}$ where the states with $S^{z}=M+1$ and $M$ become degenerate 
is given by

$$
h_{c+}(N)=E_{0}(M+1, N)-E_{0}(M, N) .
$$

Similarly, the lower critical field $h_{c-}$ where the states with $S^{z}=M$ and $M-1$ become degenerate is given by

$$
h_{c-}(N)=E_{0}(M, N)-E_{0}(M-1, N) .
$$

We therefore have a finite interval $\Delta h(N)=h_{c+}(N)-h_{c-}(N)$ in which the lowest energy state with $S^{z}=M$ is the ground state of the system with $N$ sites in the presence of a field $h$. If this interval has a nonzero limit as $N \rightarrow \infty$, we have a magnetization plateau. Thus, in the thermodynamic limit, the plateau width $\Delta h / J$ is equal to $a_{1}+a_{3}-2 a_{2}$.

We will now quote our numerical results for $J / J^{\prime}=1 / 3$. For a rung magnetization of $m_{s}=1 / 2$, i.e., $M=N / 6$, we found the three slopes $a_{i}$ to be equal to $3.77,-0.02$ and -1.93 ; see Fig. 22 . This gives the upper and lower critical fields to be

$$
\begin{aligned}
h_{c+} / J=a_{1}-a_{2} & =3.79, \quad h_{c-} / J=a_{2}-a_{3}=1.91 \\
\Delta h / J & =\left(h_{c+}-h_{c-}\right) / J=1.88 .
\end{aligned}
$$

This is a sizeable plateau width. For a rung magnetization of $m_{s}=1$, we found the $a_{i}$ to be equal to $4.97,-0.24$ and -5.43 . Thus the upper and lower critical fields are

$$
h_{c+} / J=5.21, \quad h_{c-} / J=5.19, \quad \Delta h / J=0.02 .
$$

Finally, for a rung magnetization of $m_{s}=0$, we need the energies of states with $M=0$ and $M= \pm 1$. Since the last two states must have the same energy, we have $a_{1}=a_{3}$ and it is sufficient to plot only $E_{0}(0, N)$ and $E_{0}(1, N)$ vs $1 / N$. We found $a_{1}$ and $a_{2}$ to be equal to 0.39 and 0.34 . This gives the upper and lower fields to be

$$
h_{c+} / J=0.05, \quad h_{c-} / J=-0.05, \quad \Delta h / J=0.10 .
$$

The plateau widths given in (55) and (56) are rather small. In Fig. 23, we indicate the plateau widths $\Delta h(N) / J$ as a function of $1 / N$ for $m_{s}=1 / 2,0$ and 1 .

Next, we computed various two-spin correlations for the 120-site system. These are denoted by $\left\langle\hat{S}_{a, l}^{z} \hat{S}_{b, n}^{z}\right\rangle$ and $\left\langle\hat{S}_{a, l}^{+} \hat{S}_{b, n}^{-}\right\rangle$. For the $z z$ correlations, it is convenient to subtract the product of the two separate spin densities. At $m_{s}=1 / 2$, we found that all these correlations decay very rapidly to zero as the rung separation $|l-n|$ grows. In fact, the fall offs were so fast that we were unable to compute sensible correlation 
lengths. The correlation lengths are of the order of one or two rungs as can be seen in Fig. 24.

On the other hand, for the state at $m_{s}=0$, we found that all the correlations decay quite slowly. The decays are consistent with power law fall offs of the form $A(-1)^{|l-n|} /|l-n|^{\eta}$. It is difficult to find $\eta$ very accurately since the maximum value of $|l-n|$ is only 20; this is because we fixed one site to be in the middle of the chain (to minimize edge effects), and the maximum chain length is 40 for our DMRG calculations. For $m_{s}=0$, the exponent $\eta$ for all the correlations was found to be around 1. There was no difference in the behaviors of the $z z$ and +- correlations since this was an isotropic system; $m_{s}=0$ is the ground state if the magnetic field is zero.

For the state at $m_{s}=1$ (which is the ground state only for a substantial value of the magnetic field), we found that the +- correlations again decay quite slowly consistent with a power law. The exponents $\eta$ for the different +- correlations varied from 0.61 to 0.70 with an average value of 0.66 ; see Fig. 25 for an example.

We now describe some low-temperature thermodynamic properties of the threechain system obtained using DMRG. Although DMRG is normally expected to be most accurate for targeting the lowest states in different $S^{z}$ sectors, earlier studies of mixed spin chains have shown that DMRG is quite reliable for computing low-temperature properties also [78]. There are two reasons for this; the low-lying excited states generally have a large projection onto the space of DMRG states which contains the ground state, and the low-lying excitations in one sector are usually the lowest states in nearby $S^{z}$ sectors.

We first checked that for systems with 12 sites, the results obtained using DMRG agree well with those obtained by exact diagonalization. We then used DMRG to study the magnetization, susceptibility and specific heat of 36-site systems with both $\mathrm{OBC}$ and $\mathrm{PBC}$ along the rungs. We computed these quantities using the techniques described in Eqs. (4649). The plots of magnetization vs magnetic field for various temperatures are shown in Fig. 26 for OBC along the rungs. The temperature $T$ is measured in units of $J / k_{B}$. We see that the plateau at $m_{s}=1 / 2$ disappears quite rapidly as we increase the temperature. The plateau has almost disappeared at $T=0.4$ which is substantially lower than the width $\Delta h / J=1.88$. The magnetic susceptibility is (exponentially) small at low temperatures in the region of the plateau because the magnetic excitations there are separated from the ground state by a gap. Similar results for the magnetization and susceptibility are found for the case of PBC along the rungs.

However, the specific heats at the $m_{s}=1 / 2$ plateau demonstrate an interesting difference between $\mathrm{OBC}$ and $\mathrm{PBC}$ along the rungs. While it is very small at low 
temperatures for OBC (see Fig. 27), it is not small for PBC, although it shows a plateau in the same range of magnetic fields as the magnetization itself. This observation strongly suggests that the system with $\mathrm{PBC}$ along the rungs has nonmagnetic excitations which do not contribute to the magnetization or susceptibility, but do contribute to the specific heat. Fig. 28 gives a more direct comparison between $\mathrm{OBC}$ and PBC along the rungs. Although these nonmagnetic excitations were studied by previous authors [38, 39, 94], we believe that our specific heat plots prove their existence most physically. To show these excitations even more explicitly, we present in Fig. 29 all the energy levels for a 12-site chain in the sector $S^{z}=2$ (i.e., $m_{s}=1 / 2$ ) using exact diagonalization. It is clear that the ground state is well separated from the excited states for OBC, but it is at the bottom of a band of excitations for PBC; these excitations are nonmagnetic since they have the same value of $S^{z}$ as the ground state.

We summarize our results for a three-chain spin-1/2 ladder with a large ratio of interchain coupling to intrachain coupling. There is a wide plateau with rung magnetization given by $m_{s}=1 / 2$ for both $\mathrm{OBC}$ and $\mathrm{PBC}$ along the rungs. For the case of OBC, the two-spin correlations are extremely short-ranged, and the magnetic susceptibility and specific heat are very small at low temperature in the plateau. All these are consistent with the large magnetic gap. At other values of $m$, the two-spin correlations fall off as power laws. For the case of PBC, the magnetic susceptibility is again very small at low temperature in the plateau. However the specific heat goes to zero much more slowly which dramatically shows the presence of nonmagnetic excitations.

To summarize this review, we have discussed a variety of numerical and analytical methods for studying spin clusters and quasi-one-dimensional spin systems. The methods discussed for spin clusters are directly relevant to areas of current interest such as quantum tunneling in the presence of time-dependent magnetic fields [1720,95]. One-dimensional spin systems sometimes have unusual properties (such as a disordered ground state with an excitation gap above it) which are not observed in similar models in higher dimensions. The techniques described here, particularly the DMRG method, are well-suited for studying extended systems in one dimension.

\section{Acknowledgments}

We thank H. R. Krishnamurthy, Sumathi Rao, R. Chitra, Y. Anusooya, Kunj Tandon, Siddhartha Lal, C. Raghu and Indranil Rudra for several collaborations over the years. The work on mixed spin systems was motivated by discussions with late Professor Olivier Kahn. 


\section{References}

[1] L. D. Landau and E. M. Lifshitz, Qauntum Mechanics: Non-Relativistic Theory, 3rd Ed. (Pergamon, 1977).

[2] D. C. Mattis, The Theory of Magnetism, vol. 1 (Springer-Verlag, Berlin, 1987); R. M. White, Quantum Theory of Magnetism, 2nd Ed. (Springer-Verlag, Berlin, 1983).

[3] B. Sinha and S. Ramasesha, Phys. Rev. B 48, 16410 (1993).

[4] J. N. Bray, L. V. Interrante, J. S. Jacobs and J. C. Bonner, in Extended Linear Chain Compounds, Vol. III, edited by J. S. Miller (Plenum, New York, 1982).

[5] G. T. Rado and H. Suhl eds., Magnetism, (Academic Press, New York, 1963).

[6] Z. G. Soos and S. Ramasesha, in Valence Bond Theory and Chemical Structure, edited by D. J. Klein and N. Trinajstic (Elsevier, Amsterdam, 1990) p. 81.

[7] C. Raghu, I. Rudra, D. Sen and S. Ramasesha, cond-mat/0101274, to appear in Phys. Rev. B (2001).

[8] S. Rettrup, J. Comput. Phys. 45, 100 (1982).

[9] S. Ramasesha and Z. G. Soos, J. Chem. Phys. 98, 4015 (1993).

[10] T. De Neef, Phys. Lett. A 47, 51 (1974).

[11] H. J. Blote, Physica B, 93, 93 (1978).

[12] R. Botet and R. Jullien, Phys. Rev. B 27, 613 (1983); R. Botet, R. Jullien and M. Kolb, ibid., 28, 3914 (1983).

[13] J. B. Parkinson and J. C. Bonner, Phys. Rev. B 32, 4703 (1985).

[14] A. Moreo, Phys. Rev. B 35, 8562 (1987).

[15] M. Takahashi, Phys. Rev. Lett. 62, 2313 (1989); Phys. Rev. B 48, 311 (1993).

[16] O. Golinelli, Th. Jolicoeur and R. Racaze, Phys. Rev. B 50, 3037 (1994).

[17] E. M. Chudnovsky, Science 274, 938 (1996).

[18] W. Wernsdorfer and R. Sessoli, Science 284, 133 (1999). 
[19] I. Chiorescu, W. Wernsdorfer, A. Müller, H. Bögge and B. Barbara, Phys. Rev. Lett. 84, 15 (2000).

[20] Quantum Tunneling of Magnetization - QTM'94, edited by L. Gunther and B. Barbara, NATO ASI Ser. E, Vol. 301 (Kluwer, Dordrecht, 1995); J. R. Friedman, M. P. Sarachik, J. Tejada and R. Ziolo, Phys. Rev. Lett. 76, 3830 (1996); L. Thomas, F. Lionti, R. Ballou, D. Gatteschi, R. Sessoli and B. Barbara, Nature 383, 145 (1996) and references therein; W. Wernsdorfer and R. Sessoli, Science 284, 133 (1999).

[21] D. N. Hendrickson, G. Christou, E. A. Schmitt, B. Libby, J. S. Baskin, S. Wang, H. L. Tsai, J. B. Vincent, W. P. D. Boyd, J. C. Haffman, K. Folting, Q. Li, W. E. Streib, J. Am. Chem. Soc. 114, 2455 (1992) and references therein.

[22] R. Sessoli et al., J. Am. Chem. Soc. 115, 1804 (1993).

[23] M. Hennion, L. Pardi, I. Mirebeau, E. Surad, R. Sessoli and A. Caneschi, Phys. Rev. B 56, 8819 (1997); A. A. Mukhin, V. D. Travkin, A. K. Zvezdin, S. P. Lebedev, A. Caneschi and D. Gatteschi, Europhys. Lett. 44, 778 (1998).

[24] P. A. Reynolds, E. P. Gilbert, B. N. Figgis, Inorg. Chem. 35, 545 (1996).

[25] S. M. Gorun, S. J. Lippard, Inorg. Chem. 27, 149 (1988); W. H. Armstrong, M. E. Roth, S. J. Lippard, J. Am. Chem. Soc. 109, 6318 (1987).

[26] C. D. Delfs, D. Gatteschi, L. Pardi, R. Sessoli, K. Weighardt and D. Hanke, Inorg. Chem. 32, 3099 (1993).

[27] I. Tupitsyn and B. Barbara, cond-mat/0002180, to appear in Magnetoscience From Molecules to Materials, edited by J. Miller and M. Drillon (Wiley VCH Verlag GmbH, 2000).

[28] Y. Pontillon et al., J. Am. Chem. Soc. 121, 5342 (1999).

[29] F. D. M. Haldane, Phys. Lett. 93A, 464 (1983); Phys. Rev. Lett. 50, 1153 (1983).

[30] W. J. L. Buyers, R. M. Morra, R. L. Armstrong, M. J. Hogan, P. Gerlach and K. Hirakawa, Phys. Rev. Lett. 56, 371 (1986); J. P. Renard, M. Verdaguer, L. P. Regnault, W. A. C. Erkelens, J. Rossat-Mignod and W. G. Stirling, Europhys. Lett. 3, 945 (1987); S. Ma, C. Broholm, D. H. Reich, B. J. Sternlieb and R. W. Erwin, Phys. Rev. Lett. 69, 3571 (1992). 
[31] E. Dagotto and T. M. Rice, Science 271, 618 (1996).

[32] R. S. Eccleston, T. Barnes, J. Brody and J. W. Johnson, Phys. Rev. Lett. 73, 2626 (1994).

[33] M. Azuma, Z. Hiroi, M. Takano, K. Ishida and Y. Kitaoka, Phys. Rev. Lett. 73, 3463 (1994).

[34] G. Chaboussant, P. A. Crowell, L. P. Levy, O. Piovesana, A. Madouri and D. Mailly, Phys. Rev. B 55, 3046 (1997).

[35] M. Hase, I. Terasaki and K. Uchinokura, Phys. Rev. Lett. 70, 3651 (1993); M. Nishi, O. Fujita and J. Akimitsu, Phys. Rev. B 50, 6508 (1994); G. Castilla, S. Chakravarty and V. J. Emery, Phys. Rev. Lett. 75, 1823 (1995).

[36] G. Chaboussant, Y. Fagot-Revurat, M.-H. Julien, M. E. Hanson, C. Berthier, M. Horvatic, L. P. Levy and O. Piovesana, Phys. Rev. Lett. 80, 2713 (1998).

[37] M. Oshikawa, M. Yamanaka and I. Affleck, Phys. Rev. Lett. 78, 1984 (1997).

[38] D. C. Cabra, A. Honecker and P. Pujol, Phys. Rev. Lett. 79, 5126 (1997).

[39] D. C. Cabra, A. Honecker and P. Pujol, Phys. Rev. B 58, 6241 (1998). See also the web site http://thew02.physik.uni-bonn.de/ honecker/roc.html

[40] K. Hida, J. Phys. Soc. Jpn. 63, 2359 (1994).

[41] T. Kuramoto, J. Phys. Soc. Jpn. 67, 1762 (1998).

[42] K. Totsuka, Phys. Lett. A 228, 103 (1997); Phys. Rev. B 57, 3454 (1998).

[43] T. Tonegawa, T. Nishida and M. Kaburagi, cond-mat/9712297.

[44] K. Sakai and M. Takahashi, Phys. Rev. B 57, R3201 (1998); Phys. Rev. B 57, R8091 (1998).

[45] R. Chitra and T. Giamarchi, Phys. Rev. B 55, 5816 (1997).

[46] H. J. Schulz, G. Cuniberti and P. Pieri, in Field Theories for Low Dimensional Condensed Matter Systems, edited by G. Morandi, A. Tagliacozzo and P. Sodano (Springer, Berlin, 2000); H. J. Schulz, in Proceedings of Les Houches Summer School LXI, edited by E. Akkermans, G. Montambaux, J. Pichard and J. Zinn-Justin (Elsevier, Amsterdam, 1995). 
[47] I. Affleck, in Fields, Strings and Critical Phenomena, edited by E. Brezin and J. Zinn-Justin (North-Holland, Amsterdam, 1989); I. Affleck and F. D. M. Haldane, Phys. Rev. B 36, 5291 (1987).

[48] S. Rao and D. Sen, J. Phys. Condens. Matter 9, 1831 (1997).

[49] We use the word 'phase' only for convenience to distinguish between regions with different modulations of the two-spin correlation function. Our model actually has no phase transition from Neel to spiral even at zero temperature.

[50] I. Affleck, Nucl. Phys. B 265, 409 (1986).

[51] Y. Kato and A. Tanaka, J. Phys. Soc. Jpn. 63, 1277 (1994); K. Totsuka, Y. Nishiyama, N. Hatano, and M. Suzuki, J. Phys. Condens. Matter 7, 4895 (1995).

[52] S. Rao and D. Sen, Nucl. Phys. B 424, 547 (1994).

[53] D. Allen and D. Senechal, Phys. Rev. B 51, 6394 (1995).

[54] A. O. Gogolin, A. A. Nersesyan and A. M. Tsvelik, Bosonization and Strongly Correlated Systems (Cambridge University Press, Cambridge, 1998).

[55] R. Shankar, Lectures given at the BCSPIN School, Kathmandu, 1991, in Condensed Matter and Particle Physics, edited by Y. Lu, J. Pati and Q. Shafi (World Scientific, Singapore, 1993).

[56] J. von Delft and H. Schoeller, Ann. der Physik 4, 225 (1998).

[57] J. P. Boucher and L. P. Regnault, J. de Phys. I 6, 1939 (1996).

[58] K. G. Wilson, Rev. Mod. Phys. 47, 773 (1975).

[59] J. W. Bray and S. T. Chui, Phys. Rev. B 19, 4876 (1979); J. E. Hirsh, Phys. Rev. B 22, 5259 (1980).

[60] S. R. White and R. M. Noack, Phys. Rev. Lett. 68, 3487 (1992).

[61] S. R. White, Phys. Rev. Lett. 69, 2863 (1992); Phys. Rev. B 48, 10345 (1993).

[62] R. McWeeny and B. T. Sutcliffe, Methods of Molecular Quantum Mechanics (Academic Press, London, 1969).

[63] Y. Anusooya, S. K. Pati and S. Ramasesha, J. Chem. Phys. 106, 1 (1997).

[64] S. R. White, Phys. Rev. Lett. 45, 5752 (1992). 
[65] R. Chitra, S. K. Pati, H. R. Krishnamurthy, D. Sen and S. Ramasesha, Phys. Rev. B 52, 6581 (1995); S. K. Pati, R. Chitra, D. Sen, H. R. Krishnamurthy and S. Ramasesha, Europhys. Lett. 33, 707 (1996).

[66] S. R. White and D. A. Huse, Phys. Rev. B 48, 3844 (1993); E. S. Sorensen and I. Affleck, Phys. Rev. Lett. 71, 1633 (1993).

[67] E. S. Sorensen and I. Affleck, Phys. Rev. B 49, 13235 (1994); Phys. Rev. B 49, 15771 (1994).

[68] U. Schollwöck and Th. Jolicoeur, Europhys. Lett. 30, 493 (1995); Y. Nishiyama, K. Totsuka, N. Hatano and M. Suzuki, J. Phys. Soc. Jpn. 64, 414 (1995).

[69] U. Schollwöck, Th. Jolicoeur, and T. Garel, Phys. Rev. B 53, 3304 (1996).

[70] R. J. Bursill, T. Xiang and G. A. Gehring, J. Phys. A 28, 2109 (1994); R. J. Bursill, G. A. Gehring, D. J. Farnell, J. B. Parkinson, T. Xiang and C. Zeng, J. Phys. C 7, 8605 (1995).

[71] S. R. White, R. M. Noack and D. J. Scalapino, Phys. Rev. Lett. 73, 886 (1994); M. Azzouz, L. Chen and S. Moukouri, Phys. Rev. B 50, 6223 (1994).

[72] T. Narushima, T. Nakamura and S. Takada, J. Phys. Soc. Jpn. 64, 4322 (1995); U. Schollwöck and D. Ko, Phys. Rev. B 53, 240 (1996).

[73] S. J. Qin, T. K. Ng and Z. B. Su, Phys. Rev. B 52, 12844 (1995).

[74] H. B. Pang, H. Akhlaghpour and M. Jarrel, Phys. Rev. B 53, 5086 (1996).

[75] K. A. Hallberg, Phys. Rev. B 52, R9827 (1995).

[76] S. K. Pati, S. Ramasesha, Z. Shuai and J. L. Brédas, Phys. Rev. B 59, 14827 (1999).

[77] L. J. Caron and S. Moukouri, Phys. Rev. Lett. 77, 4640 (1996).

[78] S. K. Pati, S. Ramasesha and D. Sen, Phys. Rev. B 55, 8894 (1997); J. Phys. Condens. Matt. 9, 8707 (1997).

[79] L. G. Caron and S. Moukouri, Phys. Rev. Lett. 76, 4050 (1996).

[80] T. Nishino and K. Okunishi, J. Phys. Soc. Jpn. 64, 4084 (1995).

[81] T. Nishino and K. Okunishi, J. Phys. Soc. Jpn. 65, 891 (1996). 
[82] T. Tonegawa, M. Kaburagi, N. Ichikawa, and I. Harada, J. Phys. Soc. Jpn. 61, 2890 (1992).

[83] S. Ramasesha and Z. G. Soos, Solid State Commun. 46, 509 (1983).

[84] S. Rao and D. Sen, Phys. Rev. B 48, 12763 (1993).

[85] B. Sutherland, Phys. Rev. B 12, 3795 (1975).

[86] For a review, see M. Steiner, J. Villain and C. G. Windsor, Adv. Phys. 25, 88 (1976).

[87] O. Kahn, Structure and Bonding 68, 89 (Berlin).

[88] O. Kahn, Y. Pei, M. Verdaguer, J. P. Renard and J. Sletten, J. Am. Chem. Soc. 110, 782 (1988); P. Van Koningsbruggen, O. Kahn, K. Nakatani, Y. Pei, J. P. Renard, M. Drillon and P. Leggol, Inorg. Chem. 29, 3325 (1990).

[89] O. Kahn, Adv. Inorg. Chem. 43, 179 (1995).

[90] K. A. Hallberg, P. Horsch and G. Martinez, Phys. Rev. B 52, R719 (1995); R. J. Bursill, T. Xiang and G. A. Gehring, J. Phys. A, 28, 2109 (1994); Y. Kato and A. Tanaka, J. Phys. Soc. Jpn. 63, 1277 (1994).

[91] O. Kahn, Molecular Magnetism (VCH, New York, 1993).

[92] M. Takahashi, Phys. Rev. B 36, 3791 (1987).

[93] K. Tandon, S. Lal, S. K. Pati, S. Ramasesha and D. Sen, Phys. Rev. B 59, 396 (1999).

[94] K. Kawano and M. Takahashi, J. Phys. Soc. Jpn. 66, 4001 (1997).

[95] I. Rudra, S. Ramasesha and D. Sen, Phys. Rev. B 64, 014408 (2001). 
Table 1: Low-Lying states of $\mathrm{Mn}_{12} \mathrm{Ac}$, relative to the ground state for the parameter in question. Entries in parenthesis in cases A, B and C correspond to the effective Hamiltonian results of Sessoli et al. [22]. Case D corresponds to the parameters suggested by Chudnovsky [17]. The parameters corresponding to different cases are: case (A) $J_{1}=225 \mathrm{~K}, J_{2}=90 \mathrm{~K}, J_{3}=90 \mathrm{~K}, J_{4}=0 \mathrm{~K}$; case (B) $J_{1}=225 \mathrm{~K}$, $J_{2}=90 \mathrm{~K}, J_{3}=93.8 \mathrm{~K}, J_{4}=0 \mathrm{~K}$; case $(\mathrm{C}) J_{1}=225 \mathrm{~K}, J_{2}=90 \mathrm{~K}, J_{3}=86.2 \mathrm{~K}, J_{4}=0 \mathrm{~K}$; case (D) $J_{1}=215 \mathrm{~K}, J_{2}=85 \mathrm{~K}, J_{3}=-85 \mathrm{~K}, J_{4}=-45 \mathrm{~K}$; case (E) $J_{1}=215 \mathrm{~K}, J_{2}=85 \mathrm{~K}, J_{3}=85 \mathrm{~K}$, $J_{4}=-64.5 \mathrm{~K}$. All the energies are in $\mathrm{K}$.

\begin{tabular}{|c|c|c|c|c|c|c|}
\hline Case A & Case B & Case C & \multicolumn{2}{|c|}{ Case D } & \multicolumn{2}{|c|}{ Case E } \\
\hline state $\mathrm{S} \quad \mathrm{E}(\mathrm{K})$ & state $\mathrm{S} \quad \mathrm{E}(\mathrm{K})$ & state $\mathrm{S} \quad \mathrm{E}(\mathrm{K})$ & state & $\mathrm{S} \quad \mathrm{E}(\mathrm{K})$ & state & $\mathrm{S} \mathrm{E}(\mathrm{K})$ \\
\hline $\begin{array}{lrl}{ }^{e} B & 0 & 0.0 \\
& (8) & \\
& \end{array}$ & $\begin{array}{lrr}{ }^{e} B & 0 & 0.0 \\
& (0) & \end{array}$ & $\begin{array}{ccc}{ }^{e} B & 6 & 0.0 \\
& (10) & \end{array}$ & ${ }^{e} A$ & $\begin{array}{ll}10 & 0.0\end{array}$ & ${ }^{e} A$ & $10 \quad 0.0$ \\
\hline $\begin{array}{ccc}{ }^{o} E & 1 & 10.8 \\
& (9) & (6.4) \\
& (10) & (6.4)\end{array}$ & $\begin{array}{rrr}{ }^{o} E & 1 & 16.2 \\
& (8) & (1.4)\end{array}$ & $\begin{array}{rrr}{ }^{o} E & 1 & 15.5 \\
& (8) & (2.7)\end{array}$ & ${ }^{o} E$ & $\begin{array}{ll}9 & 223\end{array}$ & ${ }^{o} E$ & $\begin{array}{ll}9 & 35.1\end{array}$ \\
\hline $\begin{array}{lrr}{ }^{o} B & 1 & 19.8 \\
& (0) & (6.8)\end{array}$ & 120.0 & $\begin{array}{lrr}{ }^{o} B & 1 & 19.6 \\
& (9) & (5.0)\end{array}$ & ${ }^{o} B$ & $\begin{array}{ll}9 & 421.2\end{array}$ & ${ }^{e} B$ & $\begin{array}{ll}8 & 62.1\end{array}$ \\
\hline $\begin{array}{lll}{ }^{e} A & 2 & 24.7\end{array}$ & 230.5 & 223.8 & ${ }^{o} B$ & $\begin{array}{ll}9 & 425.1\end{array}$ & ${ }^{o} E$ & $\begin{array}{ll}7 & 82.4\end{array}$ \\
\hline 339.0 & $4 \quad 58.4$ & 128.8 & ${ }^{e} B$ & $8 \quad 439.5$ & ${ }^{e} A$ & $\begin{array}{ll}6 & 99.7\end{array}$ \\
\hline 249.9 & 260.9 & 653.6 & ${ }^{e} B$ & 8443.7 & ${ }^{e} B$ & $\begin{array}{ll}0 & 102.0\end{array}$ \\
\hline $4 \quad 57.1$ & 364.3 & $\begin{array}{ll}6 & 54.4\end{array}$ & ${ }^{e} B$ & 8458.1 & ${ }^{e} A$ & 2121.0 \\
\hline 857.8 & 280.0 & $\begin{array}{ll}8 & 57.2\end{array}$ & ${ }^{o} A$ & $\begin{array}{ll}11573.4 \\
\end{array}$ & ${ }^{0} B$ & 1133.3 \\
\hline 257.8 & $\begin{array}{ll}3 & 88.1 \\
\end{array}$ & $\begin{array}{ll}2 & 63.0\end{array}$ & ${ }^{o} E$ & $\begin{array}{ll}9 & 583.8\end{array}$ & ${ }^{e} E$ & $\begin{array}{ll}2 & 177.1 \\
\end{array}$ \\
\hline 378.4 & $\begin{array}{ll}6 & 88.3\end{array}$ & 377.0 & ${ }^{e} E$ & $\begin{array}{ll}8 & 632.8\end{array}$ & ${ }^{o} A$ & $\begin{array}{ll}3 & 211.3\end{array}$ \\
\hline 386.8 & $\begin{array}{ll}3 & 112.8\end{array}$ & $\begin{array}{ll}3 & 85.3\end{array}$ & ${ }^{o} A$ & 9640.5 & ${ }^{o} A$ & 3220.8 \\
\hline $\begin{array}{ll}6 & 105.7\end{array}$ & $5 \quad 114.6$ & 286.1 & ${ }^{e} E$ & $\begin{array}{ll}8 & 658.3\end{array}$ & ${ }^{e} E$ & $4 \quad 249.9$ \\
\hline $\begin{array}{ll}3 & 113.4\end{array}$ & $\begin{array}{ll}5 & 158.4\end{array}$ & $\begin{array}{ll}6 & 97.1\end{array}$ & ${ }^{e} A$ & $\begin{array}{ll}8 & 767.1\end{array}$ & ${ }^{0} B$ & $5 \quad 278.5$ \\
\hline $\begin{array}{ll}4 & 117.3\end{array}$ & 1165.2 & $\begin{array}{ll}6 & 98.2\end{array}$ & ${ }^{e} B$ & 8807.6 & ${ }^{o} A$ & $\begin{array}{ll}7 & 332.1\end{array}$ \\
\hline $\begin{array}{ll}5 & 154.2\end{array}$ & 181.6 & $\begin{array}{ll}3 & 112.2\end{array}$ & ${ }^{e} A$ & 8815.8 & ${ }^{o} A$ & 7340.8 \\
\hline
\end{tabular}


Table 2: Energies (in unit of $\mathrm{K}$ ) of few low lying states in $\mathrm{Fe}_{8}$. The exchange constants corresponding to the various cases are: case (1) $\mathrm{J}_{1}=150 \mathrm{~K}, \mathrm{~J}_{2}=25 \mathrm{~K}, \mathrm{~J}_{3}$ $=30 \mathrm{~K}, \mathrm{~J}_{4}=50 \mathrm{~K}$; case $(2) \mathrm{J}_{1}=180 \mathrm{~K}, \mathrm{~J}_{2}=153 \mathrm{~K}, \mathrm{~J}_{3}=22.5 \mathrm{~K}, \mathrm{~J}_{4}=52.5 \mathrm{~K}$; case (3) $\mathrm{J}_{1}=195 \mathrm{~K}, \mathrm{~J}_{2}=30 \mathrm{~K}, \mathrm{~J}_{3}=52.5 \mathrm{~K}, \mathrm{~J}_{4}=22.5 \mathrm{~K}$. All the energies are in $\mathrm{K}$.

\begin{tabular}{|cc|cc|cc|}
\hline \multicolumn{2}{|c|}{ Case 1 } & \multicolumn{2}{c|}{ Case 2 } & \multicolumn{2}{c|}{ Case 3 } \\
\hline $\mathrm{S}$ & $\mathrm{E}(\mathrm{K})$ & $\mathrm{S}$ & $\mathrm{E}(\mathrm{K})$ & $\mathrm{S}$ & $\mathrm{E}(\mathrm{K})$ \\
\hline 10 & 0.0 & 10 & 0.0 & 10 & 0.0 \\
\hline 9 & 13.1 & 9 & 3.4 & 9 & 39.6 \\
\hline 8 & 27.3 & 8 & 10.2 & 9 & 54.2 \\
\hline 9 & 41.7 & 7 & 20.1 & 9 & 62.4 \\
\hline
\end{tabular}




\section{Figure Captions}

1. A schematic representation of the VB diagrams for eight spin- $1 / 2$ objects. States $\mid 1>$ and $\mid 2>$ are legal VB diagrams and $\mid 3>$ is an illegal VB diagram.

2. VB diagram for spin- $1 / 2$ objects with total spin $1 / 2(\mid 1>)$ and total spin 1 $(\mid 2>)$. Their bit representations $(0$ and 1$)$ as well as the unique integer $I_{k}$ representing them are shown. $P$ and $P^{\prime}$ are the phantom sites. $\mid 3>$ is a singlet VB diagram corresponding to two spin- 1 , a spin- $5 / 2$ and a spin-3/2 object.

3. Effect of operation by the operator $\left(\hat{\mathbf{S}}_{i} \cdot \hat{\mathbf{S}}_{j}-1 / 4\right)$ on a state with a singlet line between sites $i$ and $j$ and on a state with sites $i$ and $j$ singlet paired with two different sites $i^{\prime}$ and $j^{\prime}$.

4. A schematic diagram of the exchange interactions between the Mn ions in the $\mathrm{Mn}_{12} \mathrm{Ac}$ molecule.

5. Spin densities in the ground state $\left(\mathrm{S}=10, \mathrm{M}_{\mathrm{S}}=10\right)$ of $\mathrm{Mn}_{12} \mathrm{Ac}$ for parameter values $J_{1}=215 \mathrm{~K}, J_{2}=85 \mathrm{~K}, J_{3}=85 \mathrm{~K}$ and $J_{4}=-64.5 \mathrm{~K}$.

6. A schematic diagram of the exchange interactions between the Fe ions in the $\mathrm{Fe}_{8}$ molecule.

7. Spin density in the ground state $\left(S=10, \mathrm{M}_{\mathrm{S}}=10\right)$ of $\mathrm{Fe}_{8}$ for three different parameter values: (a) $\mathrm{J}_{1}=150 \mathrm{~K}, \mathrm{~J}_{2}=25 \mathrm{~K}, \mathrm{~J}_{3}=30 \mathrm{~K}, \mathrm{~J}_{4}=50 \mathrm{~K}$, (b) $\mathrm{J}_{1}=180 \mathrm{~K}$, $\mathrm{J}_{2}=153 \mathrm{~K}, \mathrm{~J}_{3}=22.5 \mathrm{~K}, \mathrm{~J}_{4}=52.5 \mathrm{~K}$, and (c) $\mathrm{J}_{1}=195 \mathrm{~K}, \mathrm{~J}_{2}=30 \mathrm{~K}, \mathrm{~J}_{3}=52.5 \mathrm{~K}$, $\mathrm{J}_{4}=22.5 \mathrm{~K}$.

8. Schematic picture of the frustrated and dimerized spin chain.

9. Classical phase diagram of the spin chain in the $J_{2}-\delta$ plane.

10. 'Phase' diagram for the spin- 1 chain in the $J_{2}-\delta$ plane.

11. Doubly degenerate ground states (a) and (b) of the $J-J_{2}$ chain (see Fig. 8 for $\delta=0)$ at $J_{2}=J / 2$. The solid line between sites $i$ and $j$ represents a singlet, $\left[\left|\uparrow_{i \downarrow_{j}}>-\right| \downarrow_{i} \uparrow_{j}>\right] / \sqrt{2}$.

12. 'Phase' diagram for the spin- $1 / 2$ chain in the $J_{2}-\delta$ plane. 
13. Gap $\Delta$ vs $J$ for coupled spin chains $(\delta=1)$. Spin- $1 / 2$ and spin-1 data are indicated by crosses and circles respectively.

14. Expectation values of the $z$-components of the two spins vs the unit cell index $n$ for an alternating spin chain. The upper and lower points are for the spin- $s_{1}$ and the spin- $s_{2}$ sites respectively.

15. Subtracted two-spin correlation functions as a function of distance between the two spins. (a) spin- $s_{1}$ spin- $s_{1}$ correlations, (b) spin- $s_{2}$ spin- $s_{2}$ correlations, and (c) spin- $s_{1}$ spin- $s_{2}$ correlations. In each figure, squares correspond to $(3 / 2,1)$, circles to $(3 / 2,1 / 2)$ and triangles to $(1,1 / 2)$ systems.

16. Energy difference (units of $J$ ) between the ground state and the lowest energy state with spin $S=S_{G}-1$ as a function of inverse system size. $S_{G}$ is the total spin of the ground state.

17. Excitation gap (units of $J$ ) from the ground state ( $\operatorname{spin} S=S_{G}$ ) to the state with spin $S=S_{G}+1$ as a function of the inverse system size.

18. Excitation gap (units of $J$ ) to the state with spin $S=S_{G}+1$ from the ground state $\left(S=S_{G}\right)$ as a function of $\delta$ for the dimerized alternating chain. The exponent is $1.0 \pm 0.01$ for all three systems.

19. Plot of magnetization per site as a function of temperature $T$ for four different values of the magnetic fields $B$. Squares are for $B=0.1 J / g \mu_{B}$, circles for $B=0.5 \mathrm{~J} / g \mu_{B}$, triangles for $B=\mathrm{J} / g \mu_{B}$ and diamonds for $B=2 \mathrm{~J} / g \mu_{B}$.

20. $\chi T$ ( defined in the text) per site as a function of temperature $T$ for various magnetic fields $B$. Zero field results are shown by squares, $B=0.01 \mathrm{~J} / g \mu_{B}$ by circles, $B=0.1 \mathrm{~J} / g \mu_{B}$ by triangles and $B=\mathrm{J} / g \mu_{B}$ by diamonds.

21. Specific heat per site as a function of temperature $T$ for four different values of magnetic fields $B$. Zero field data are shown by squares, $B=0.01 \mathrm{~J} / g \mu_{B}$ by circles, $B=0.1 \mathrm{~J} / g \mu_{B}$ by triangles and $B=\mathrm{J} / g \mu_{B}$ by diamonds.

22. The energy/site in units of $J$ vs $1 / N$ at the $m_{s}=1 / 2$ plateau, for the threechain ladder with $J / J^{\prime}=1 / 3$. The curves indicate quadratic fits for (a) $E_{0}(M+1, N)$, (b) $E_{0}(M, N)$, and (c) $E_{0}(M-1, N)$.

23. Plateau widths vs $1 / N$ for (a) $m_{s}=1 / 2$, (b) $m_{s}=0$, and (c) $m_{s}=1$.

24. Correlation function $<\hat{S}_{2, l}^{+} \hat{S}_{2, n}^{-}>$at the $m_{s}=1 / 2$ plateau for $J / J^{\prime}=1 / 3$. 
25. Correlation functions $<\hat{S}_{1, l}^{+} \hat{S}_{2, n}^{-}>$in the $m_{s}=1$ state for $J / J^{\prime}=1 / 3$.

26. Magnetization vs magnetic field at six different temperatures, for a 36-site system with $\mathrm{OBC}$ along the rungs and $J / J^{\prime}=1 / 3$.

27. Specific heat in units of $k_{B}$ vs magnetic field at six different temperatures, for a 36-site system with $\mathrm{OBC}$ along the rungs and $J / J^{\prime}=1 / 3$.

28. Comparisons of specific heat and susceptibility vs the magnetic field for a 36-site systems with $\mathrm{OBC}$ and $\mathrm{PBC}$ along the rungs.

29. Comparison of the energy spectra in units of $J$ of the 12 -site system with OBC and $\mathrm{PBC}$ along the rungs. The energies in the $S^{z}=2$ sector are shown for $J / J^{\prime}=1 / 3$. 

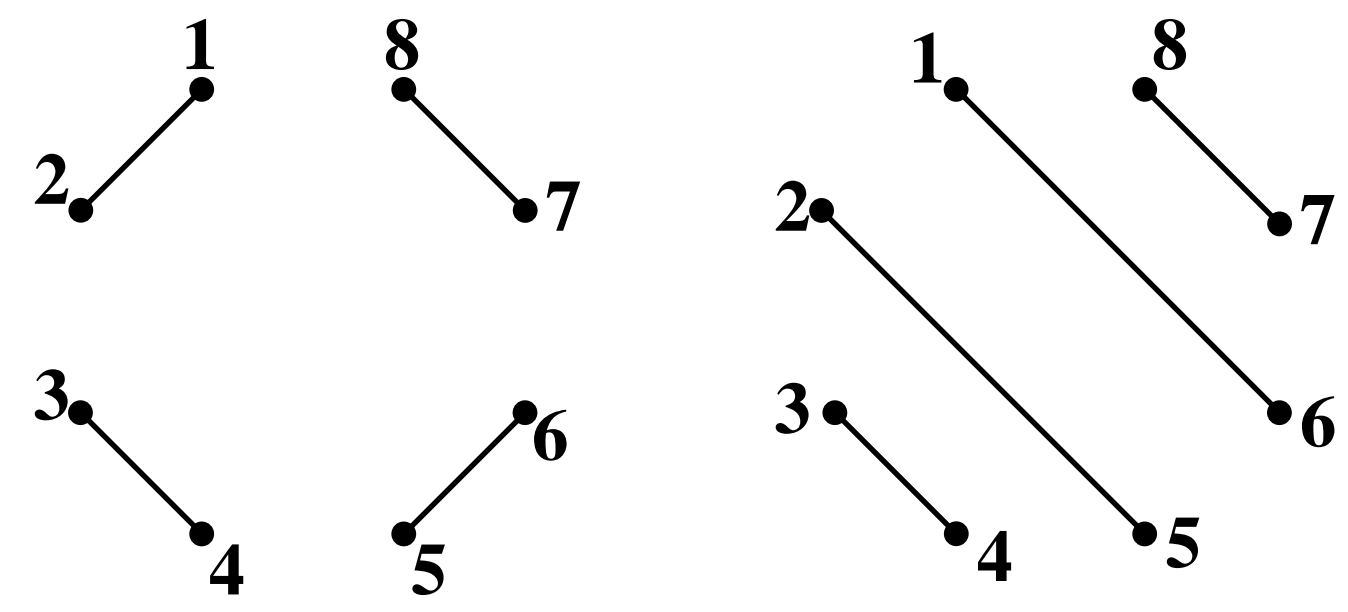

|1>

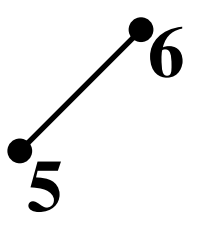

|2>

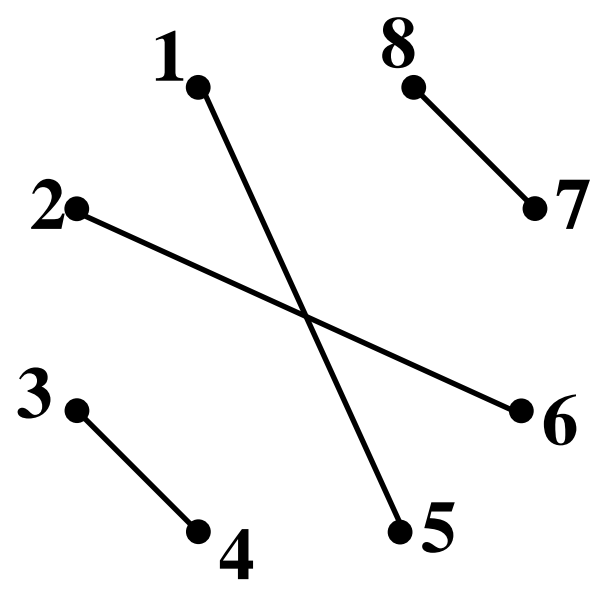

|3>

Fig. 1 

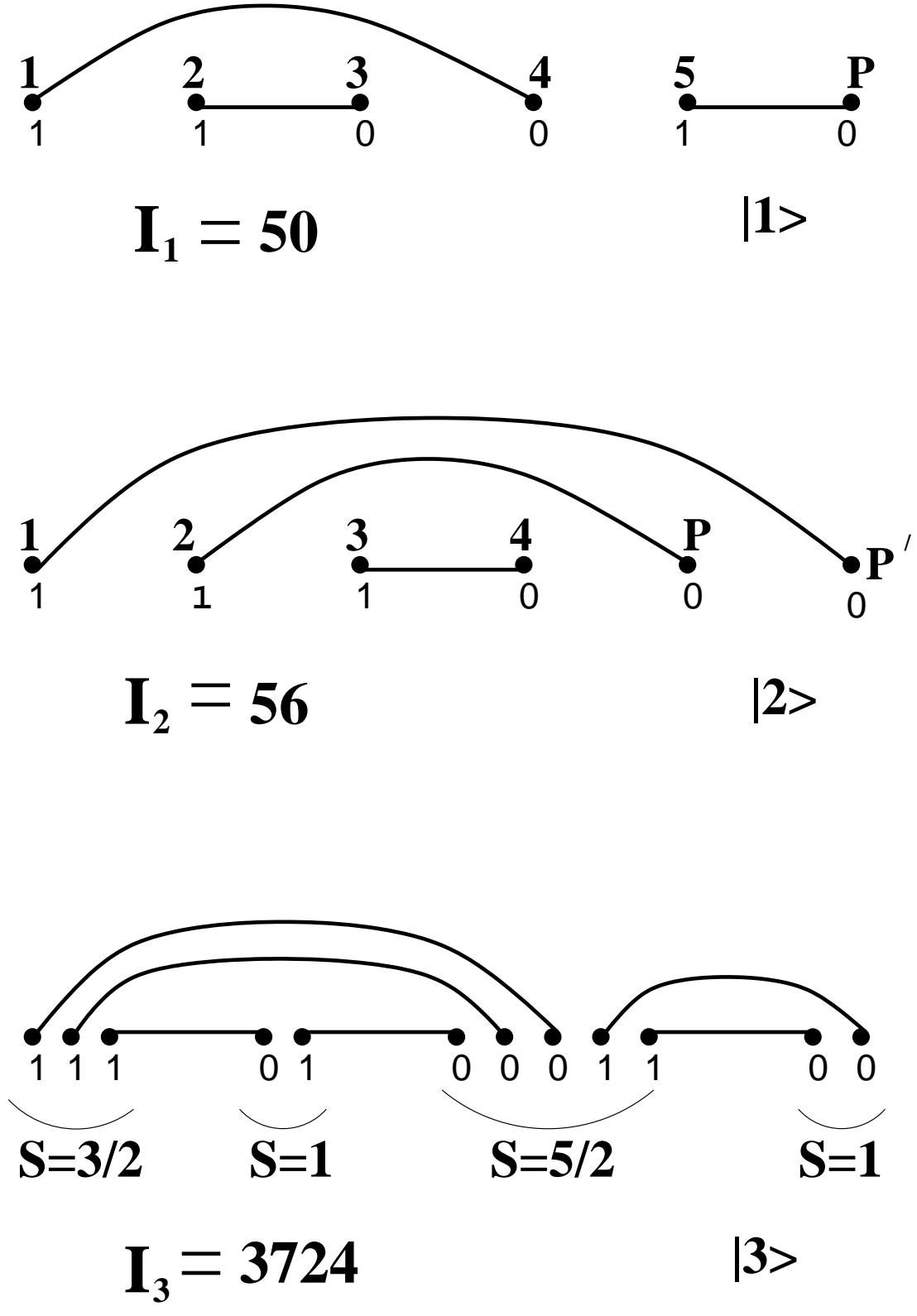

Fig. 2 


$$
\begin{aligned}
& \left(\hat{\mathbf{S}}_{\mathbf{i}} \cdot \hat{\mathbf{S}}_{\mathbf{j}}-\frac{1}{4}\right)|\mathbf{i} \quad \mathbf{j}\rangle=-\mid \begin{array}{ll}
\mathbf{i} & \mathbf{j}\rangle
\end{array}
\end{aligned}
$$

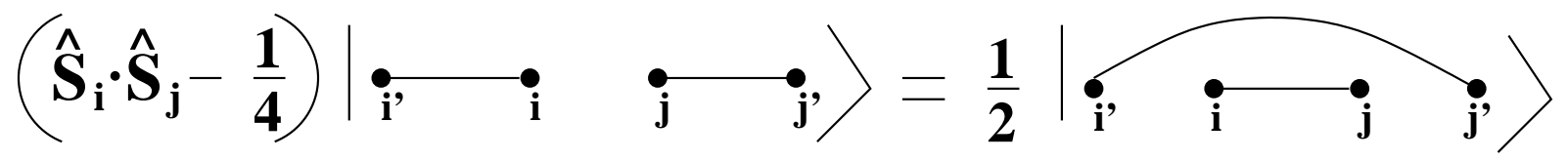

Fig. 3 


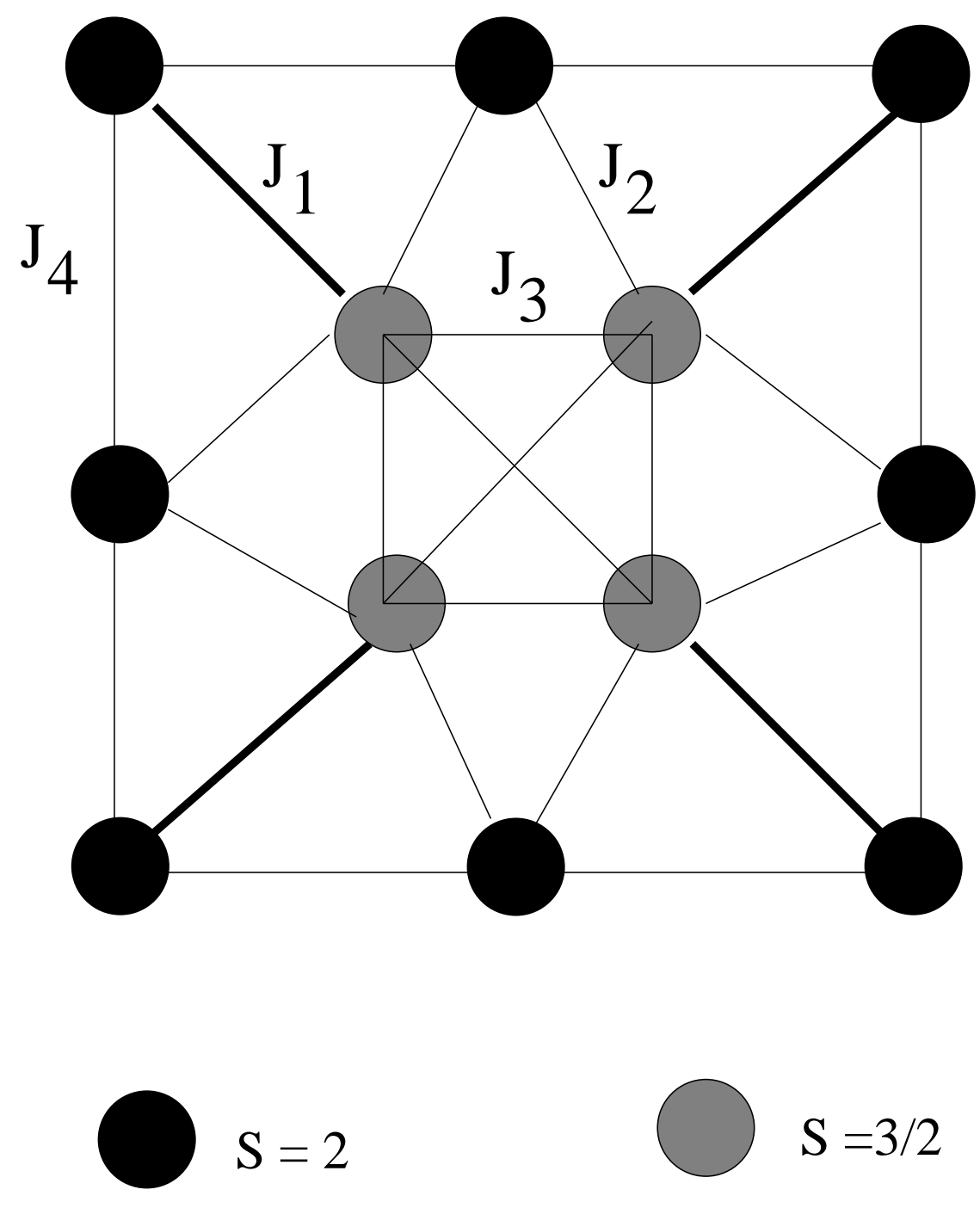

Fig. 4 


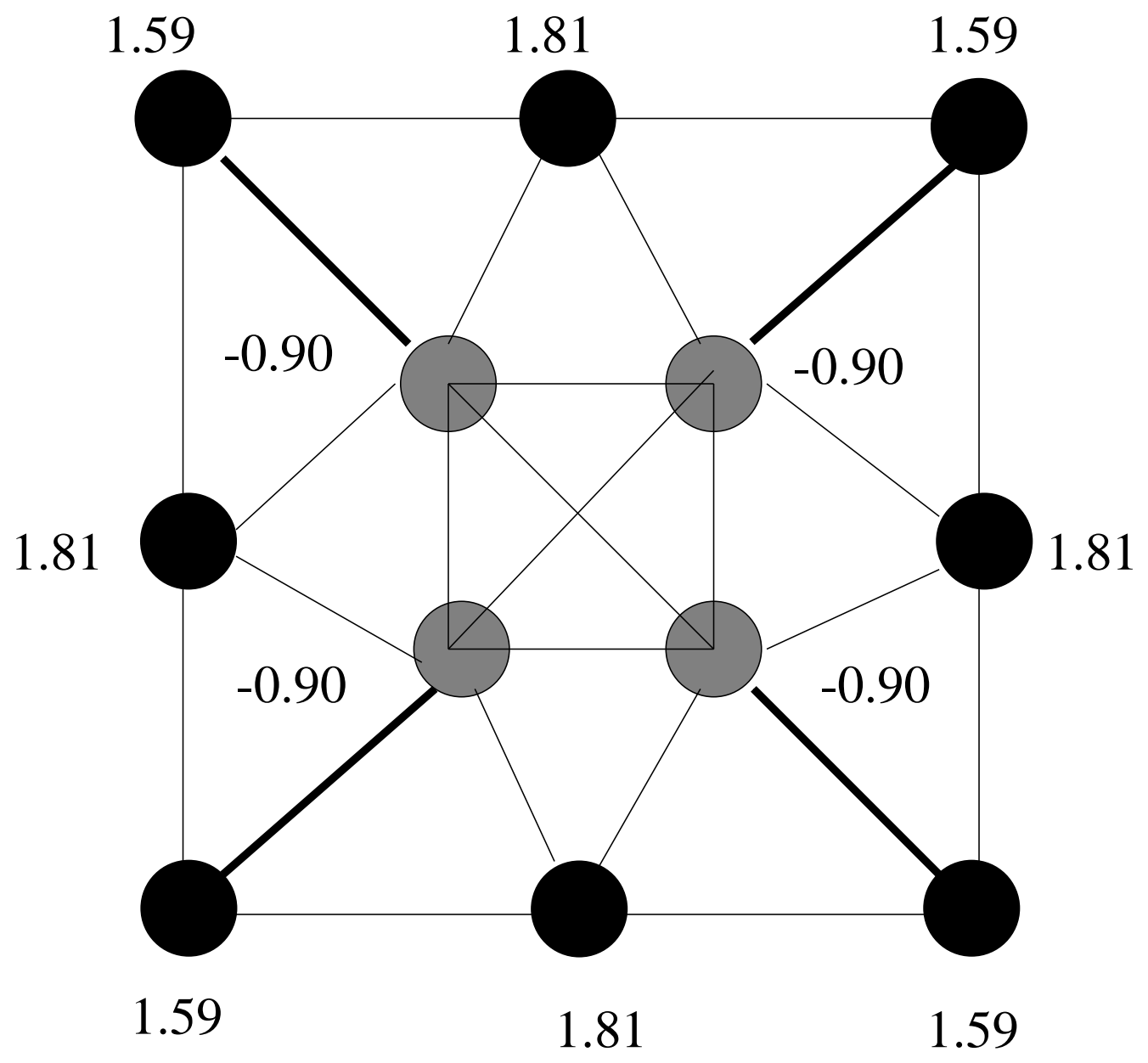

Fig. 5 


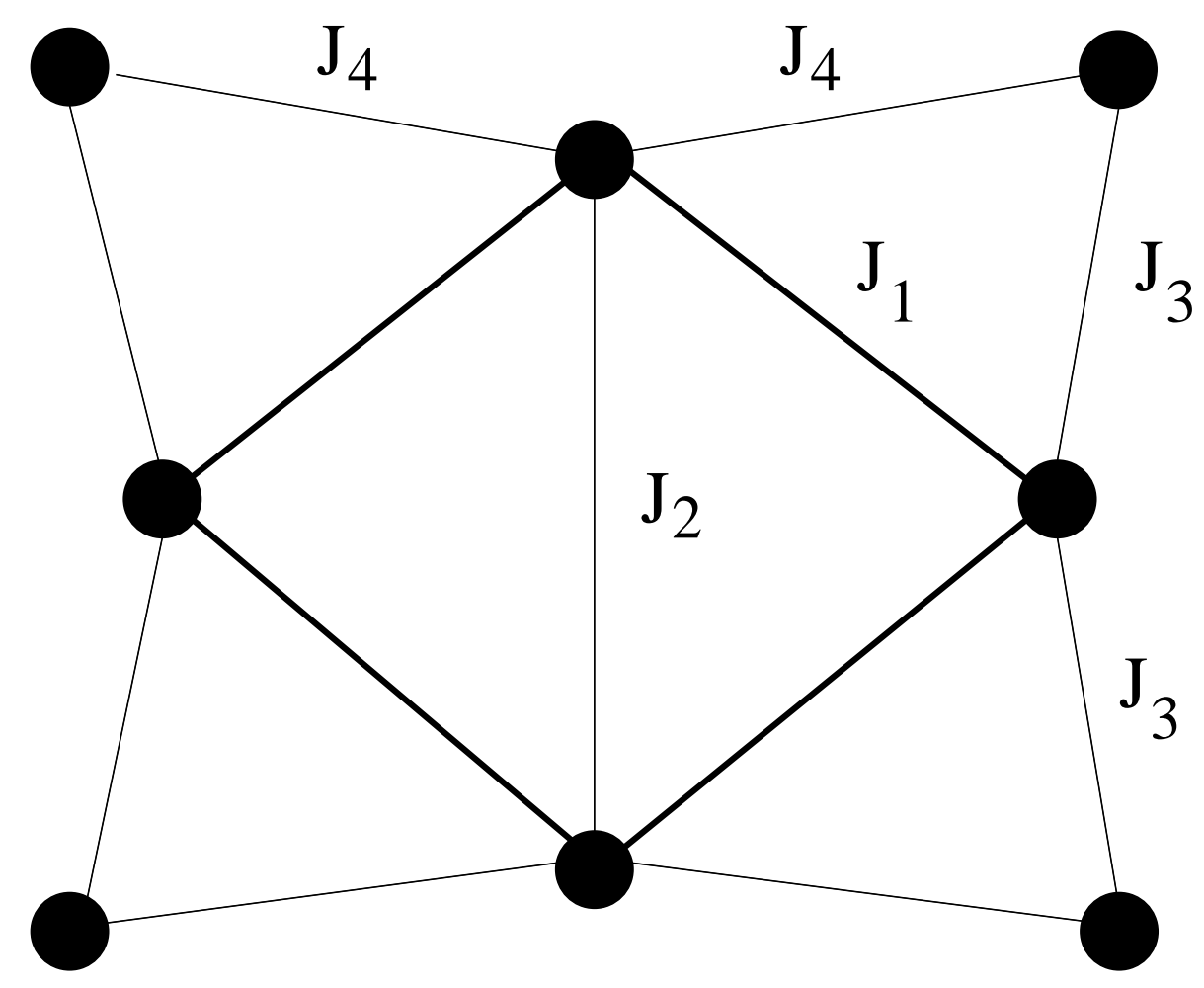

Fig. 6 


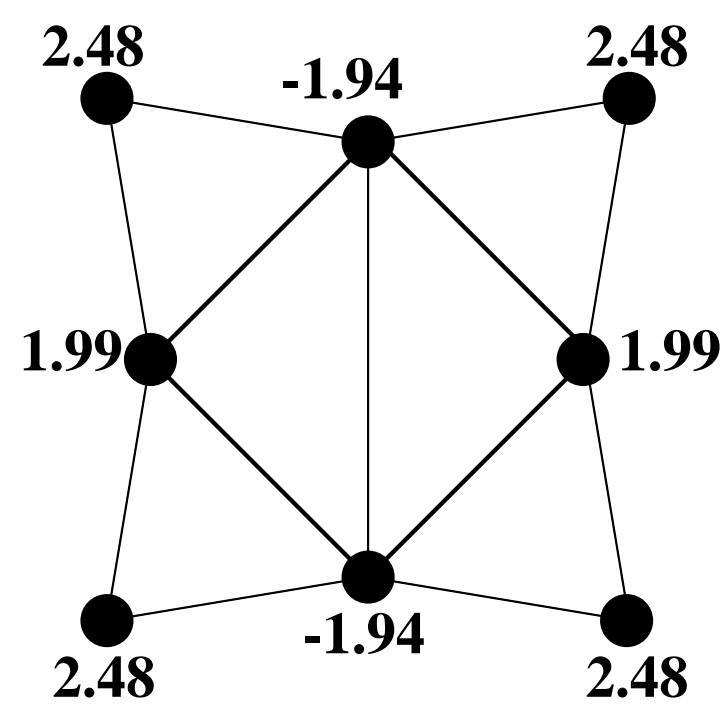

(a)

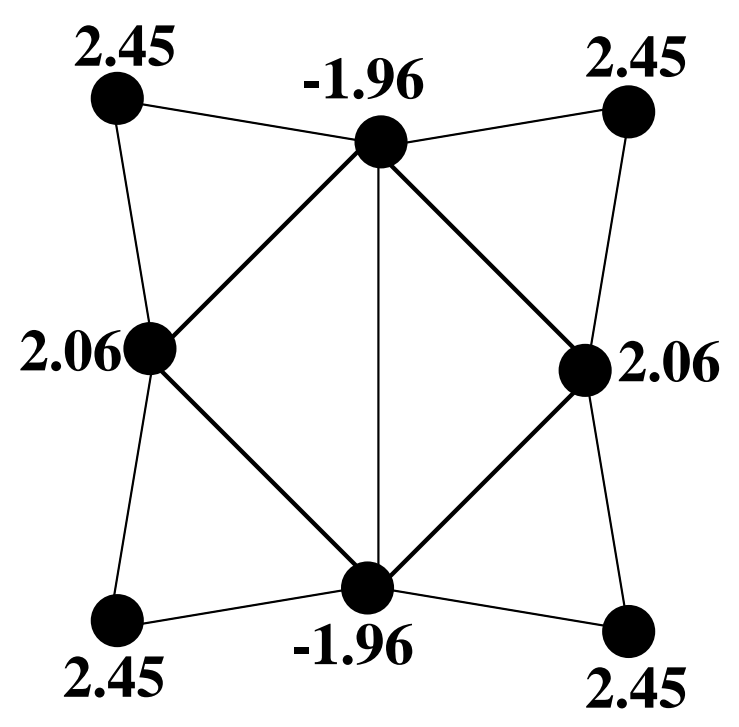

(b)

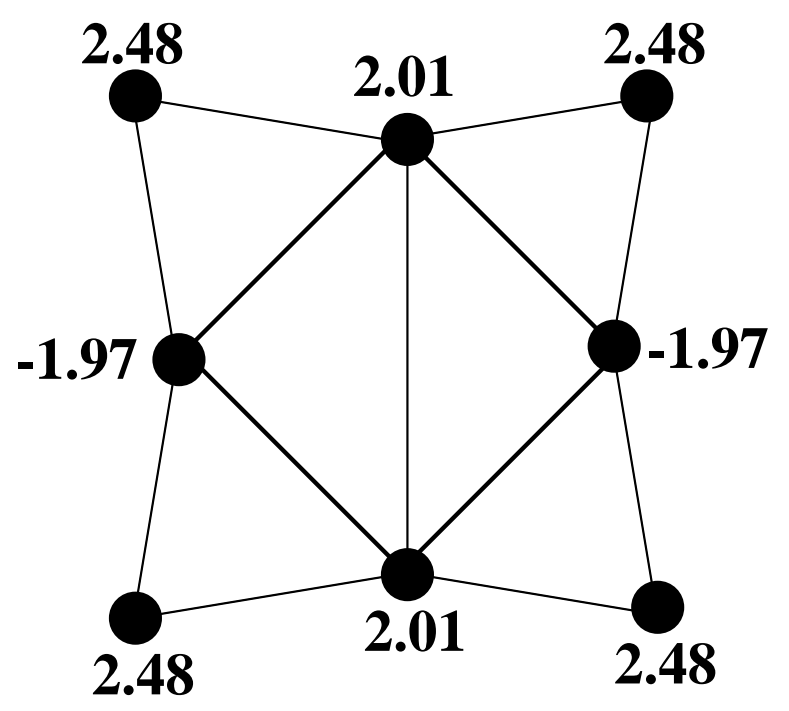

(c)

Fig. 7 


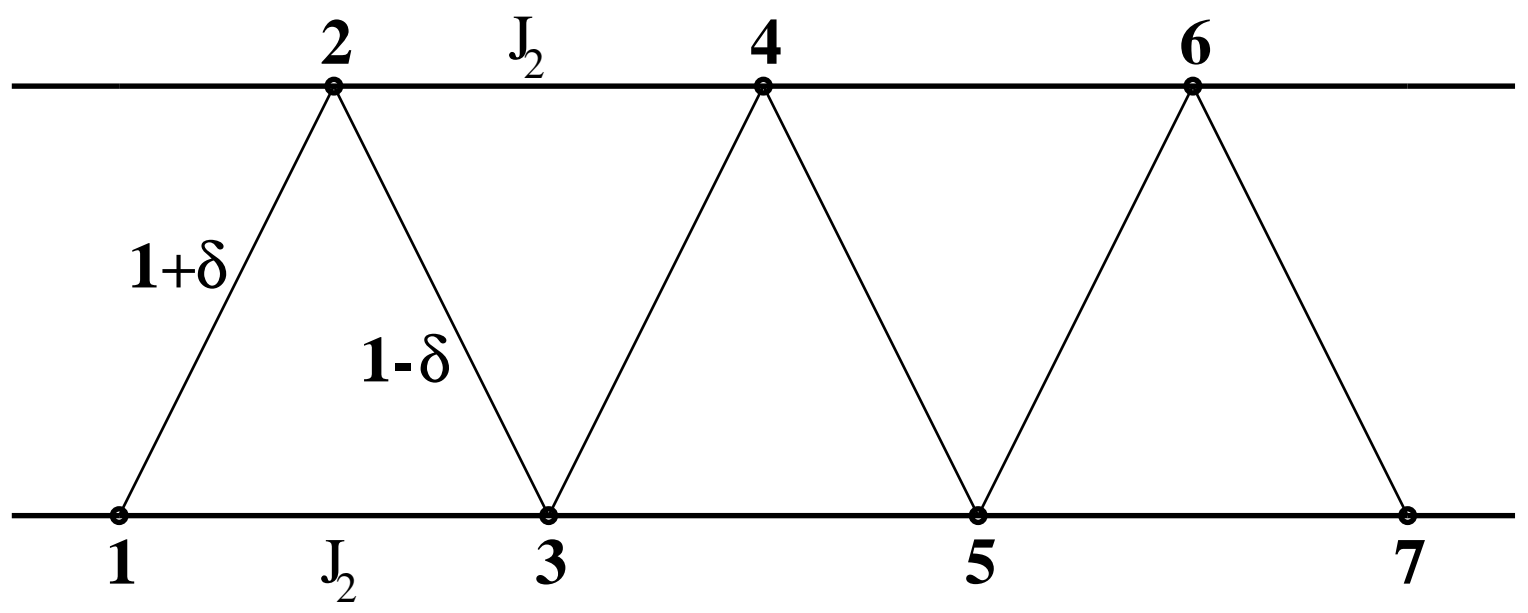

Fig. 8 


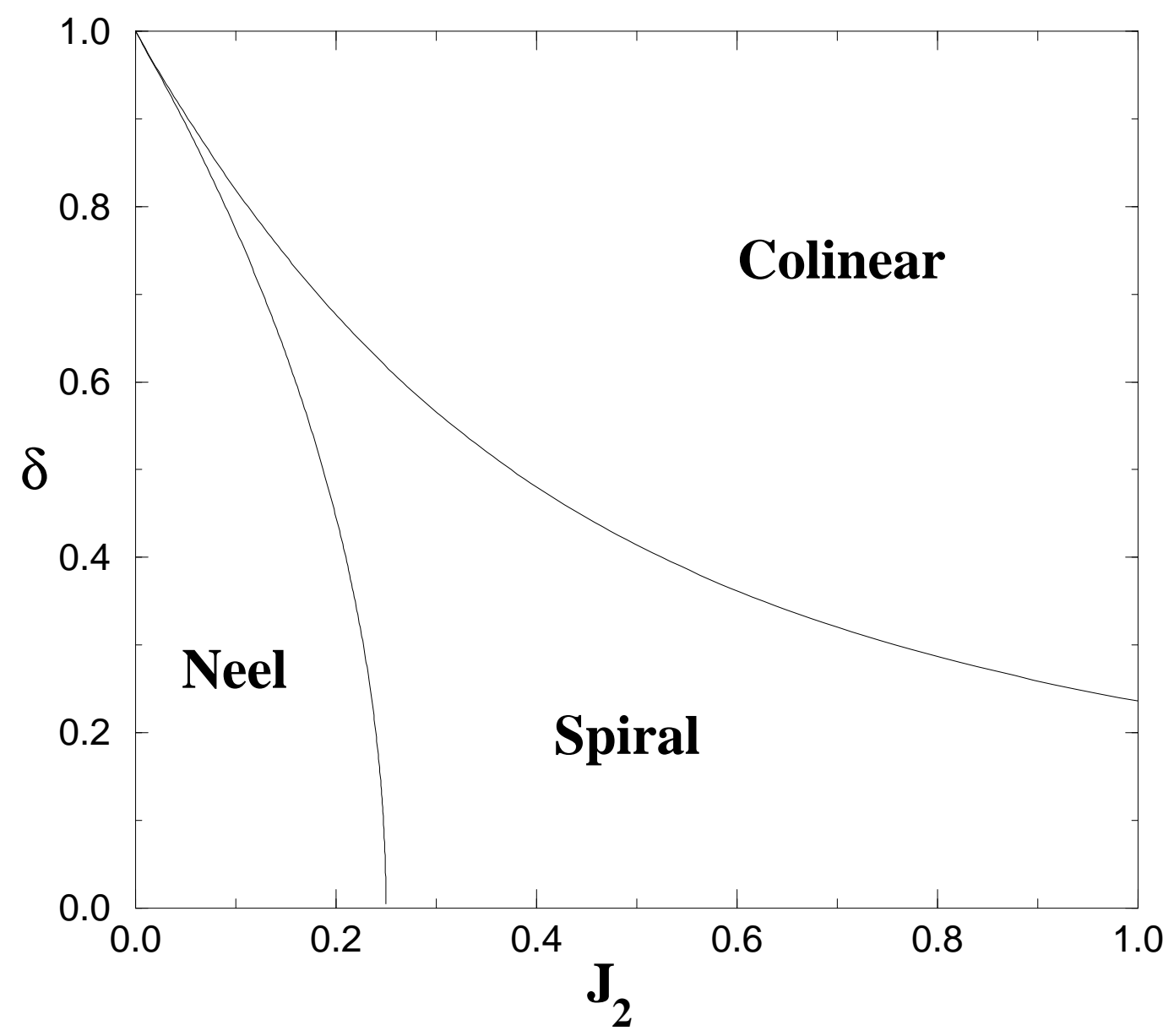

Fig. 9 


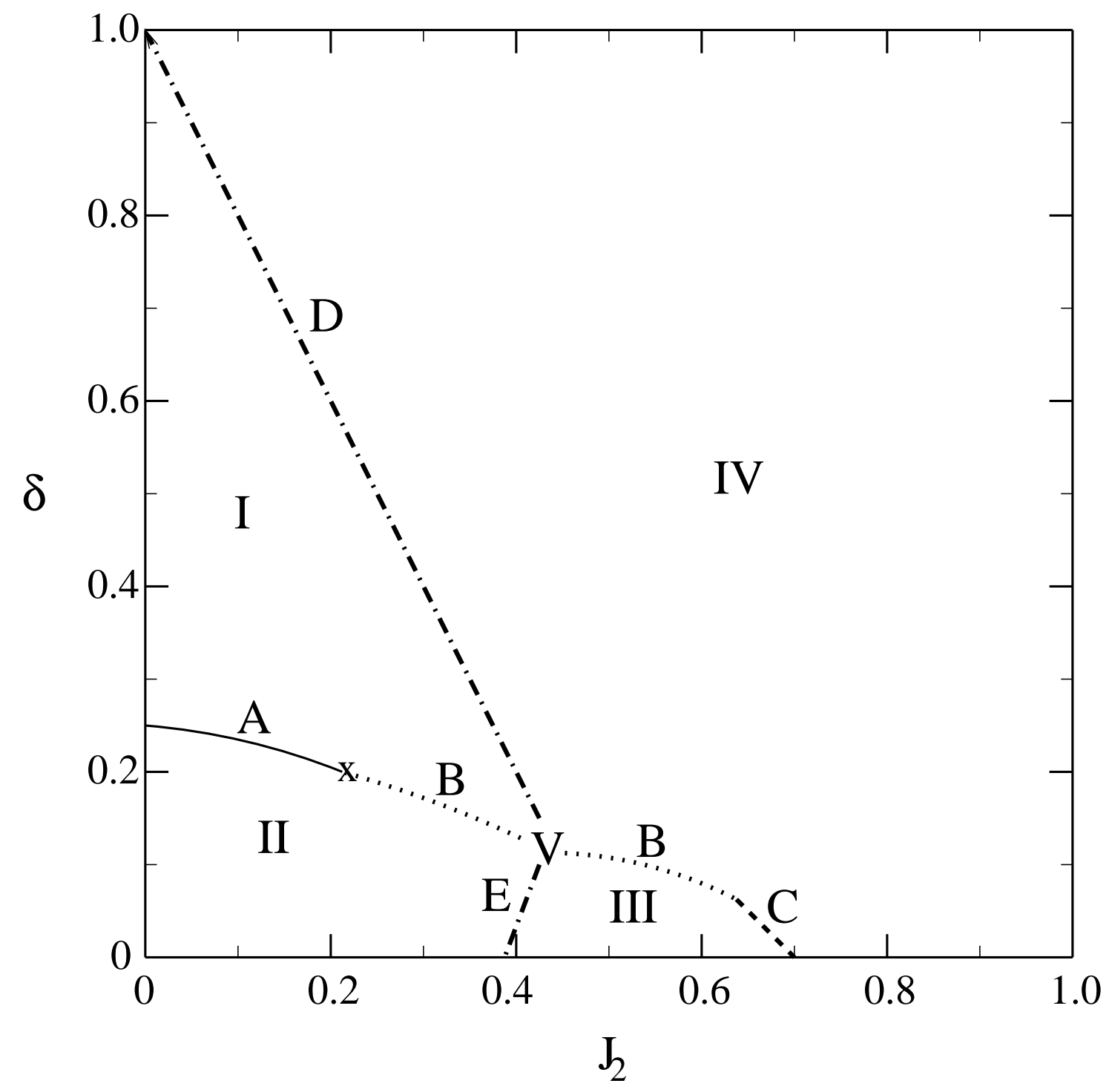

Fig. 10 

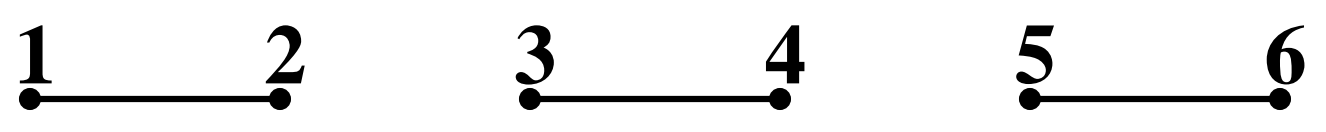

7

(a)

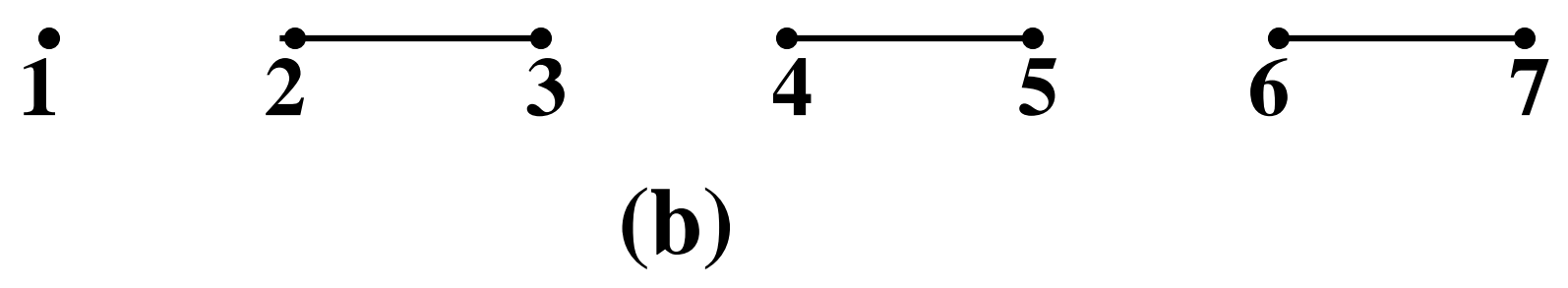

Fig. 11 


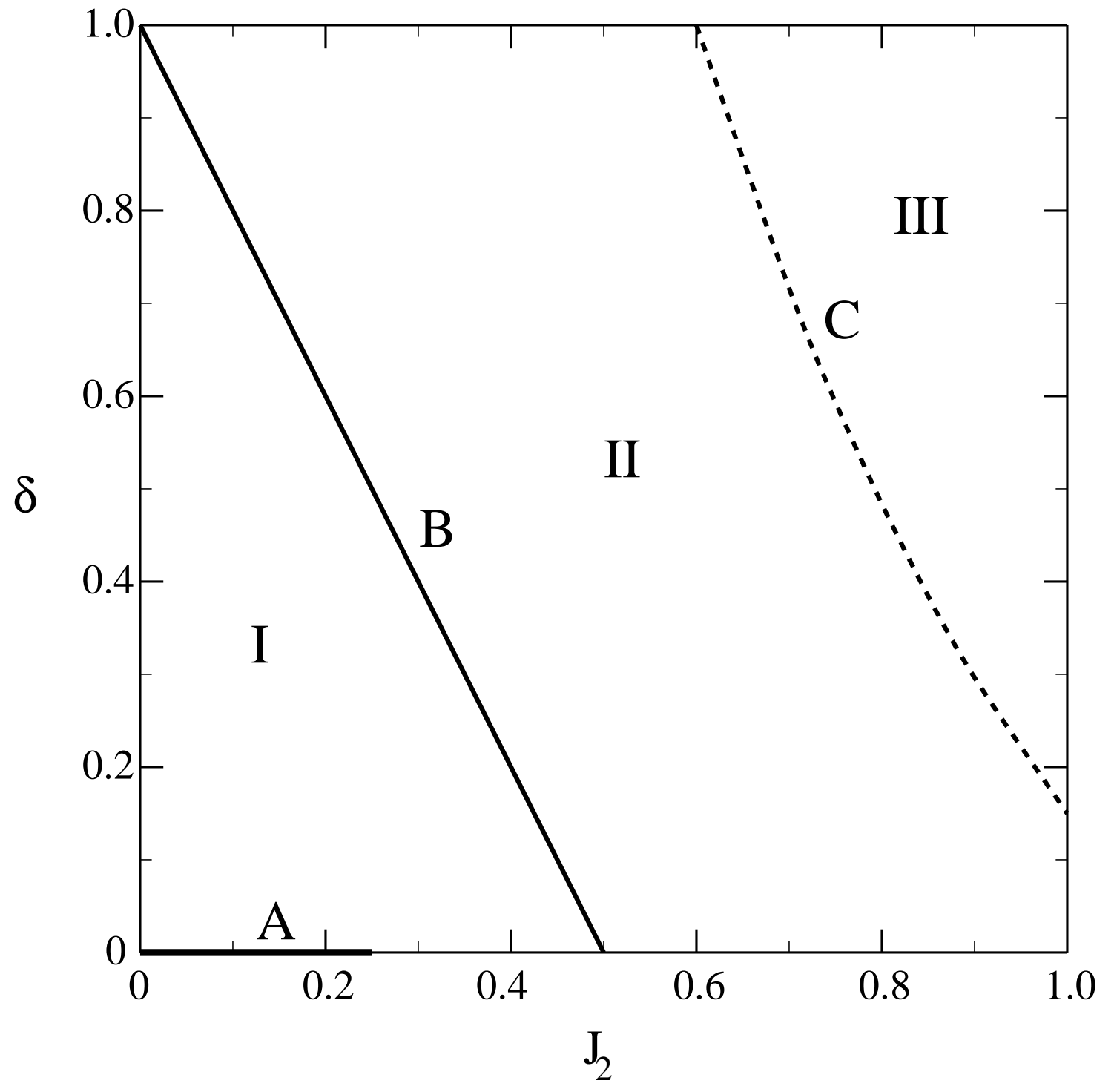

Fig. 12 


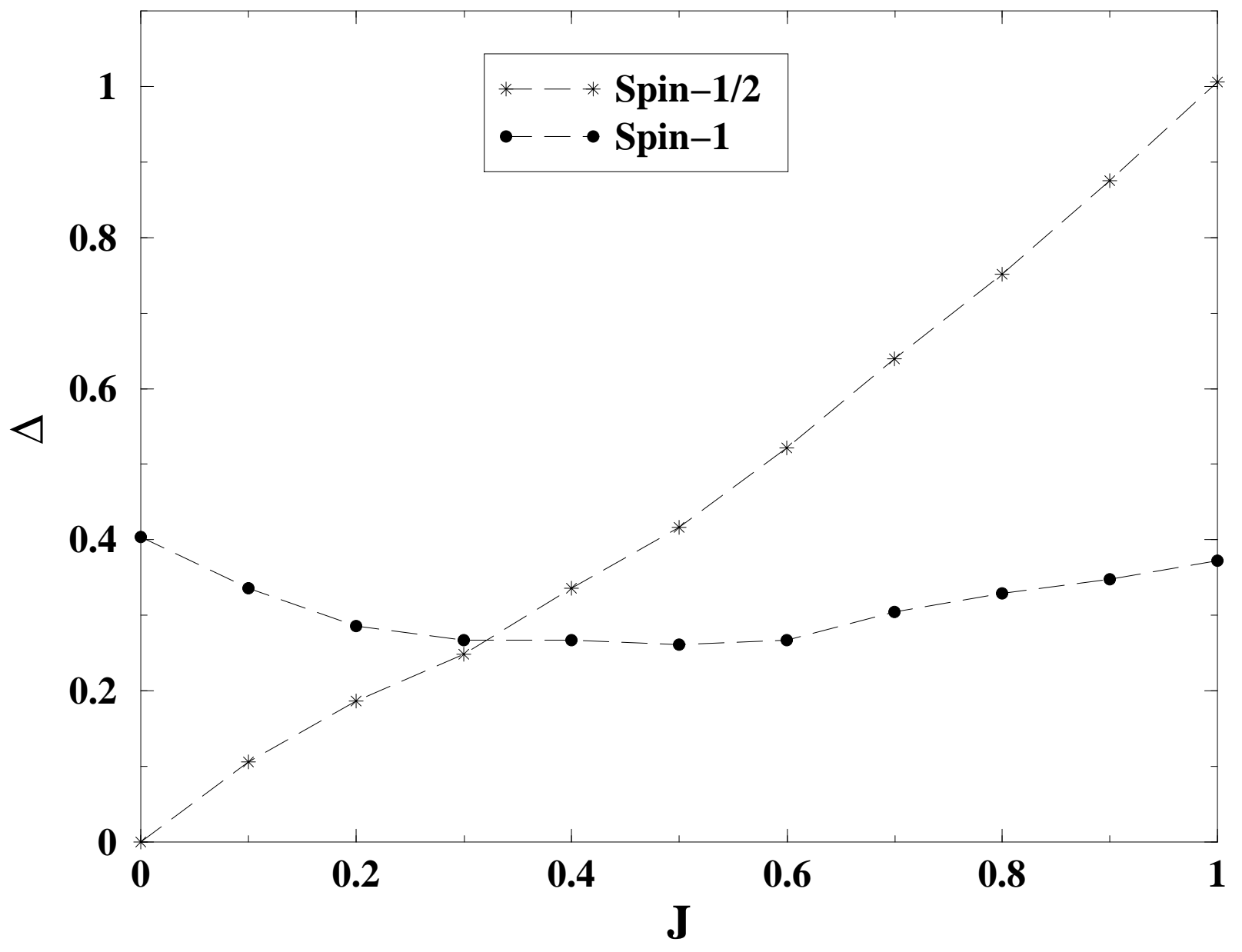

Fig. 13 

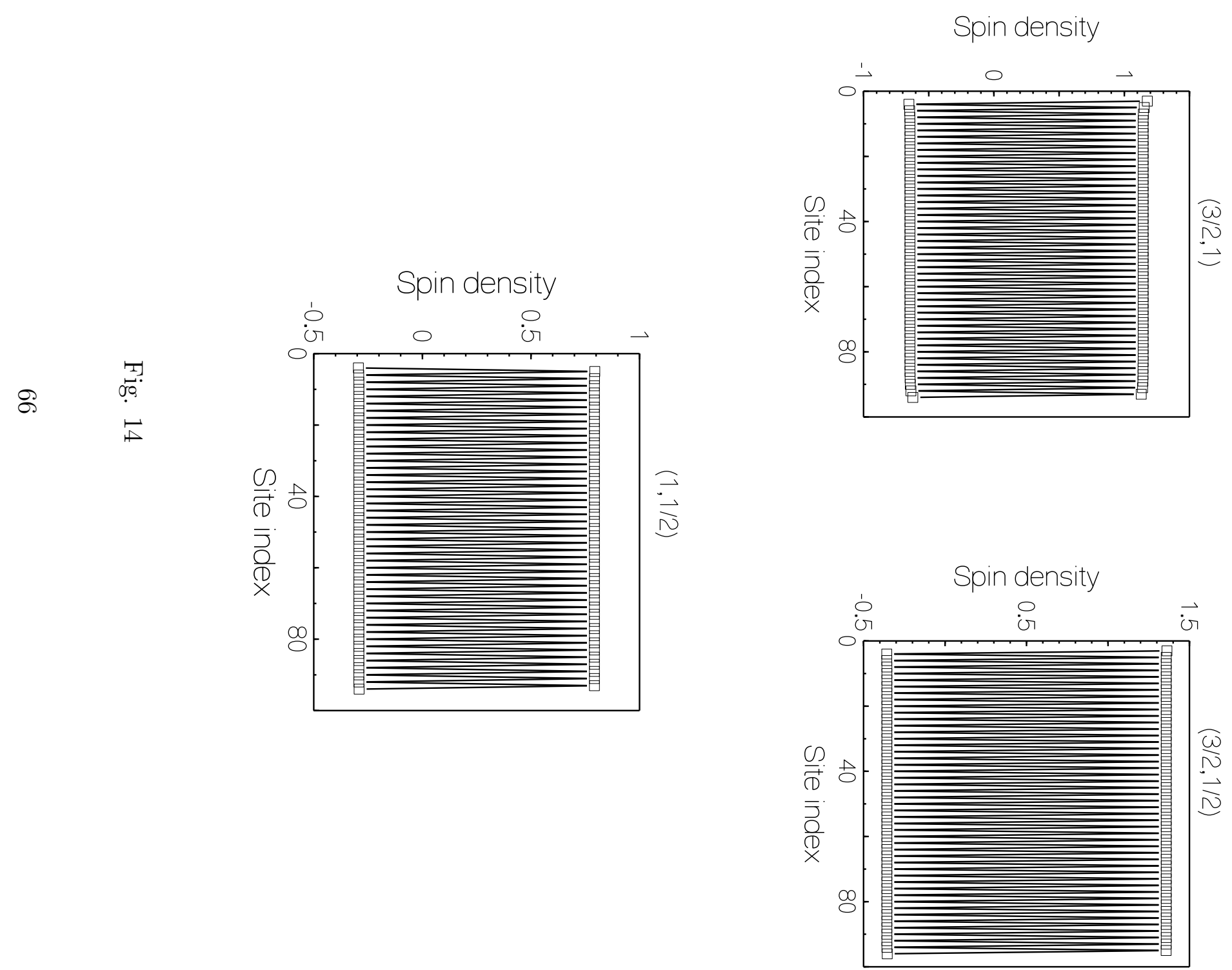


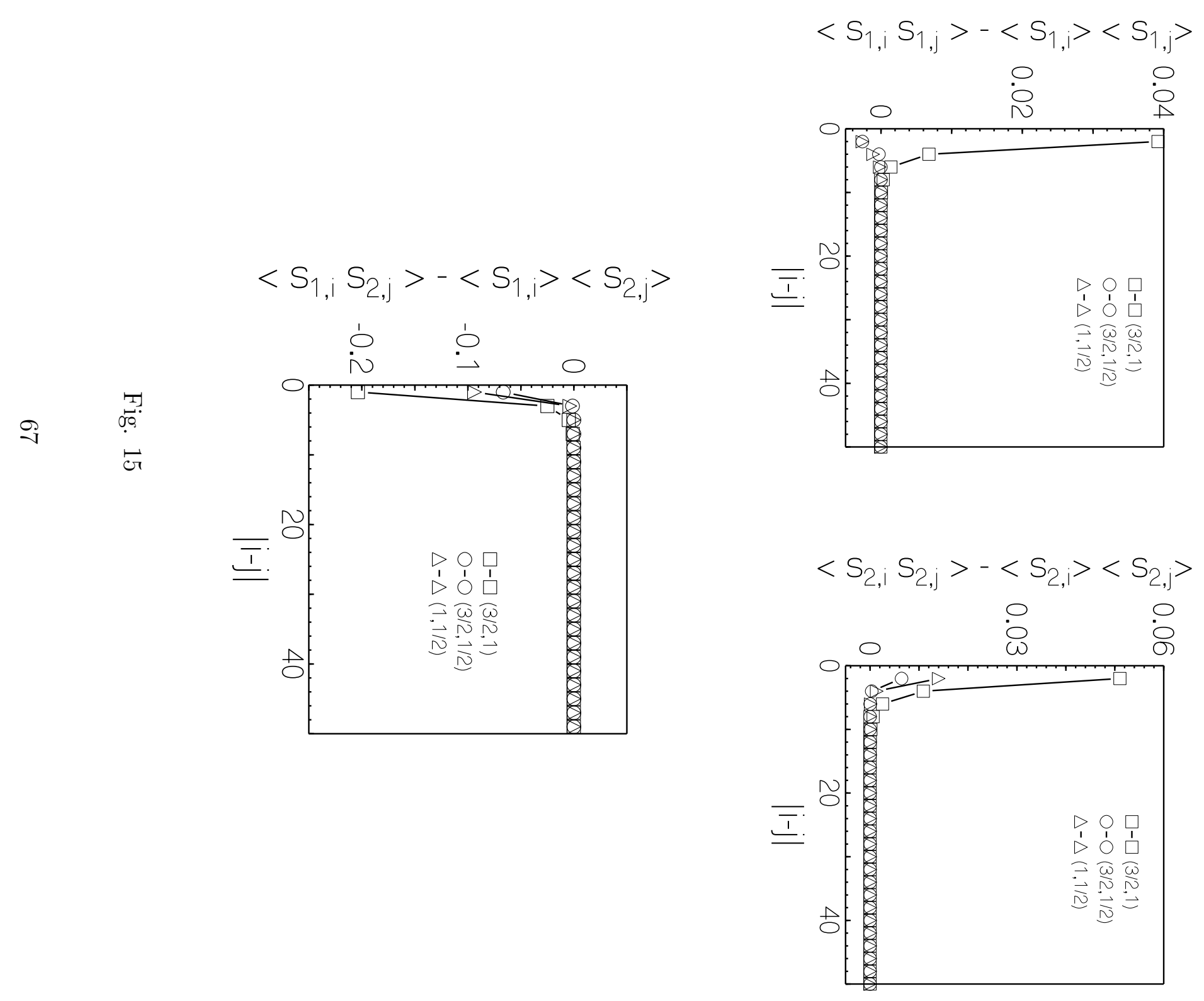




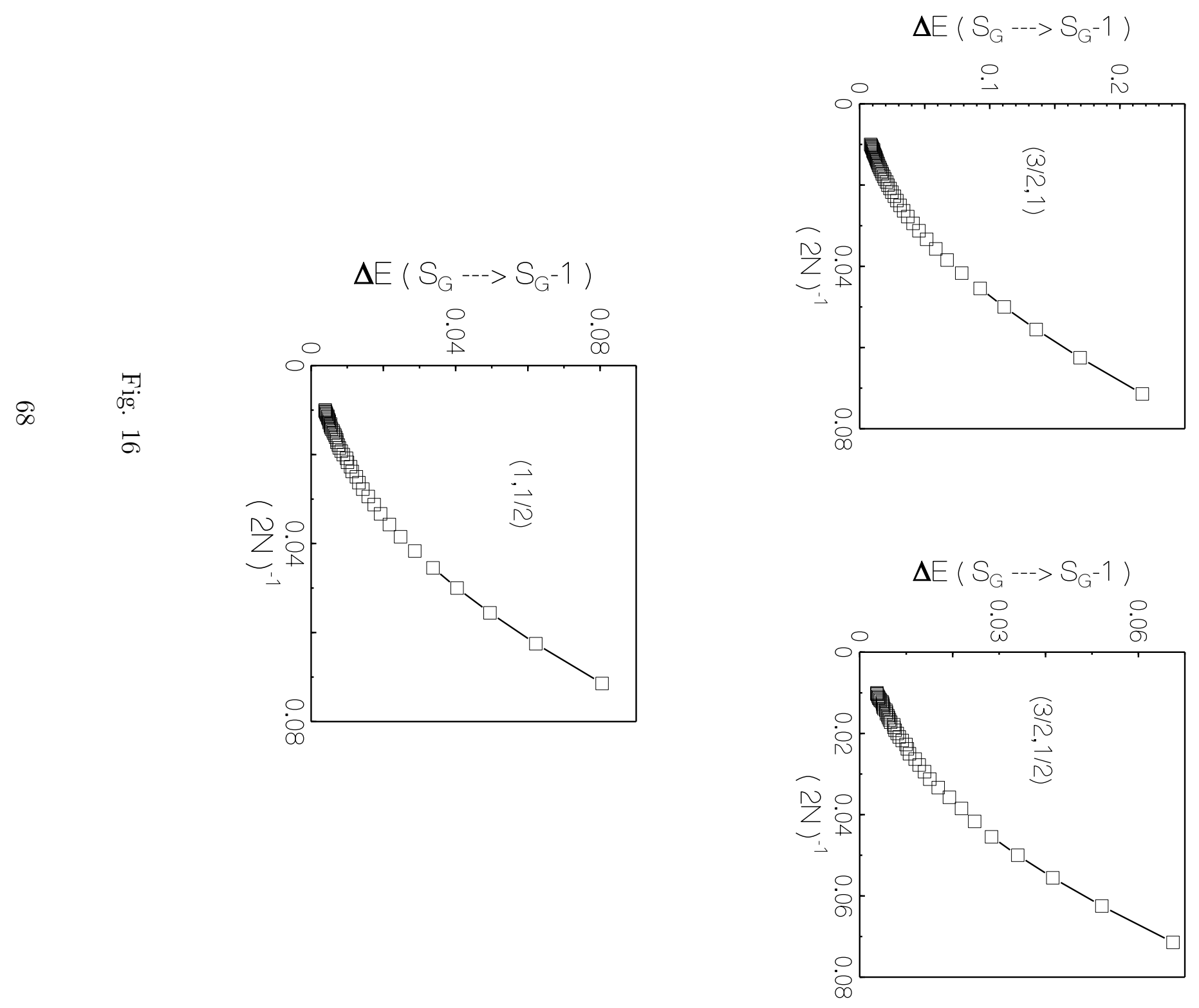




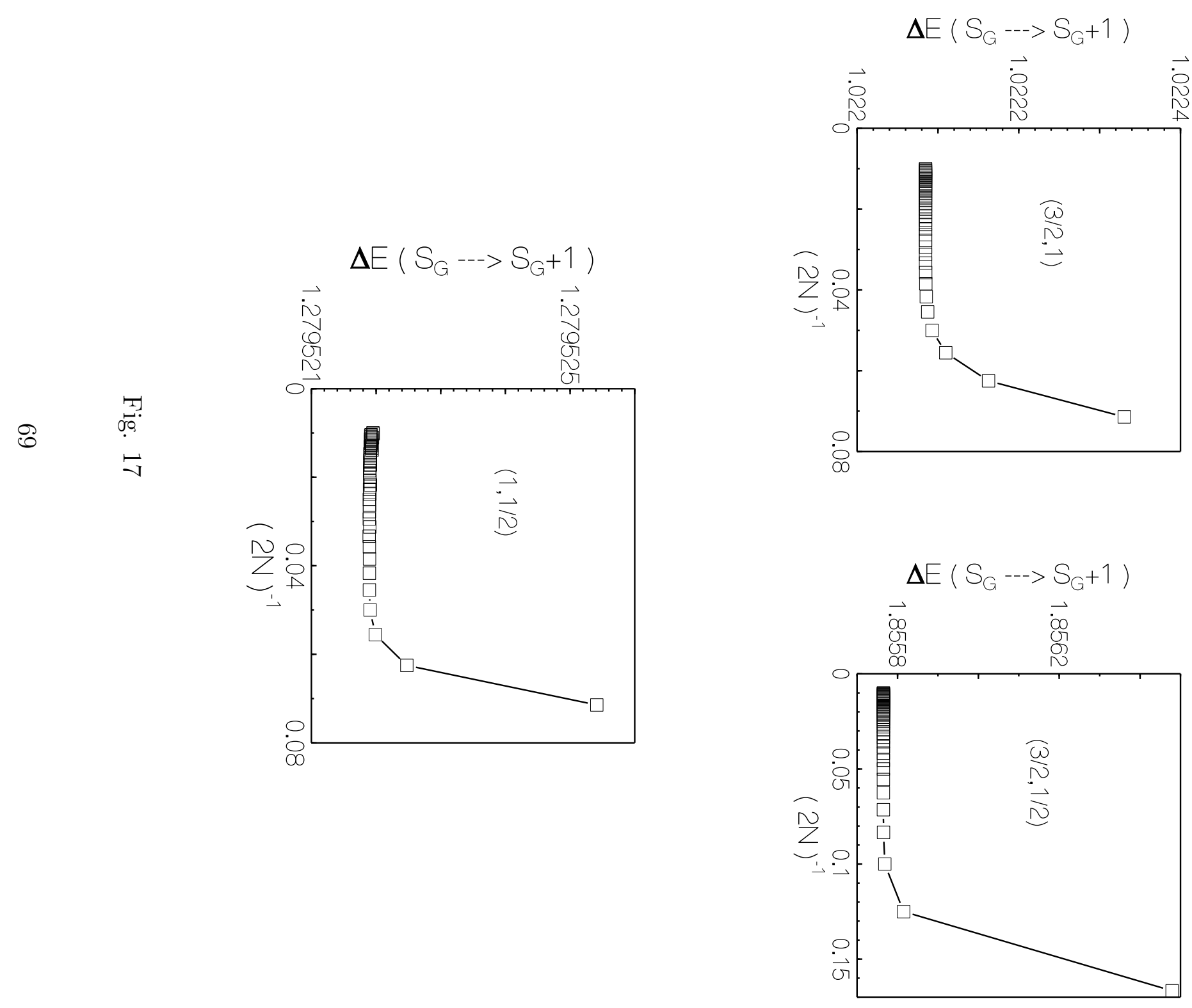




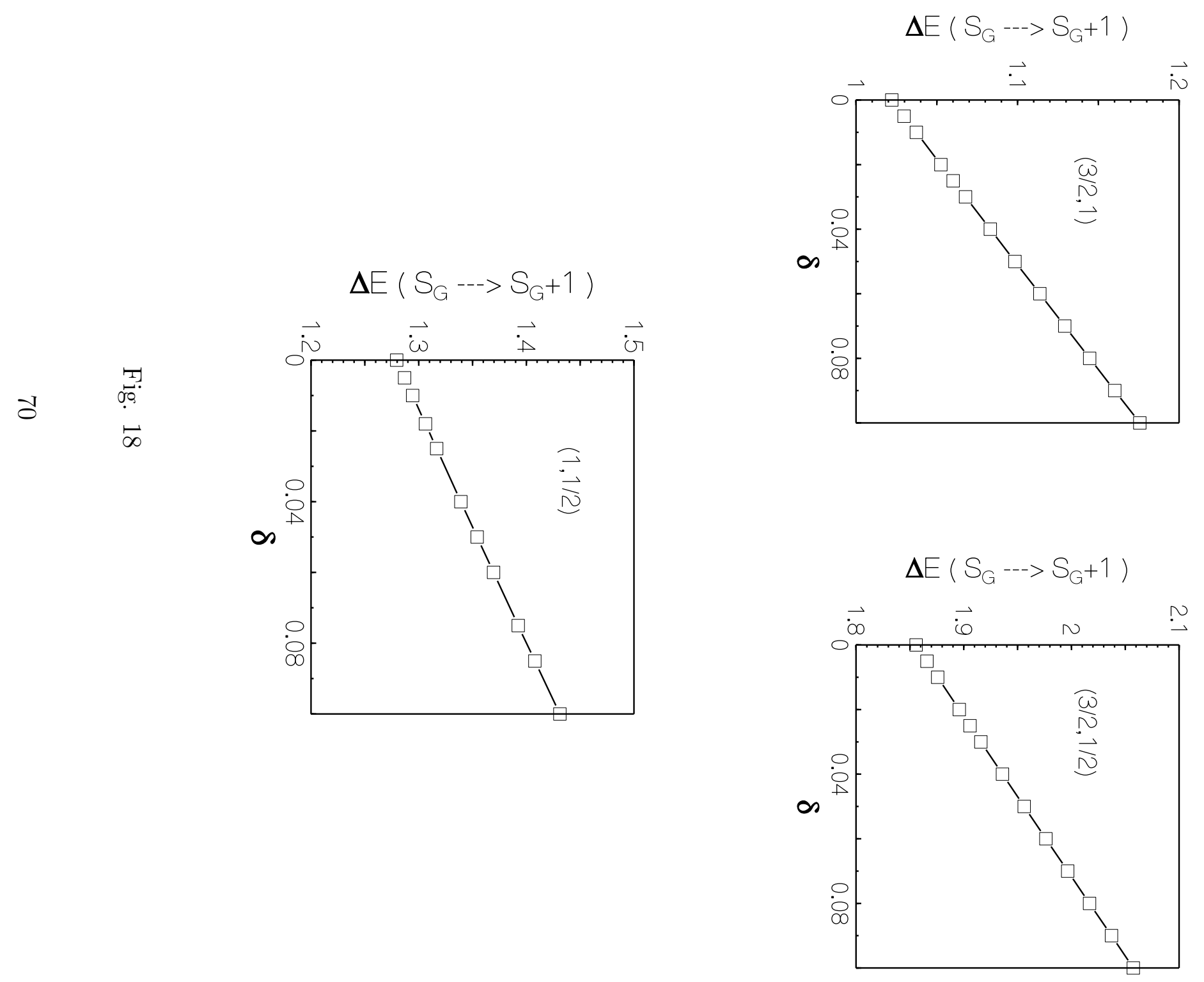




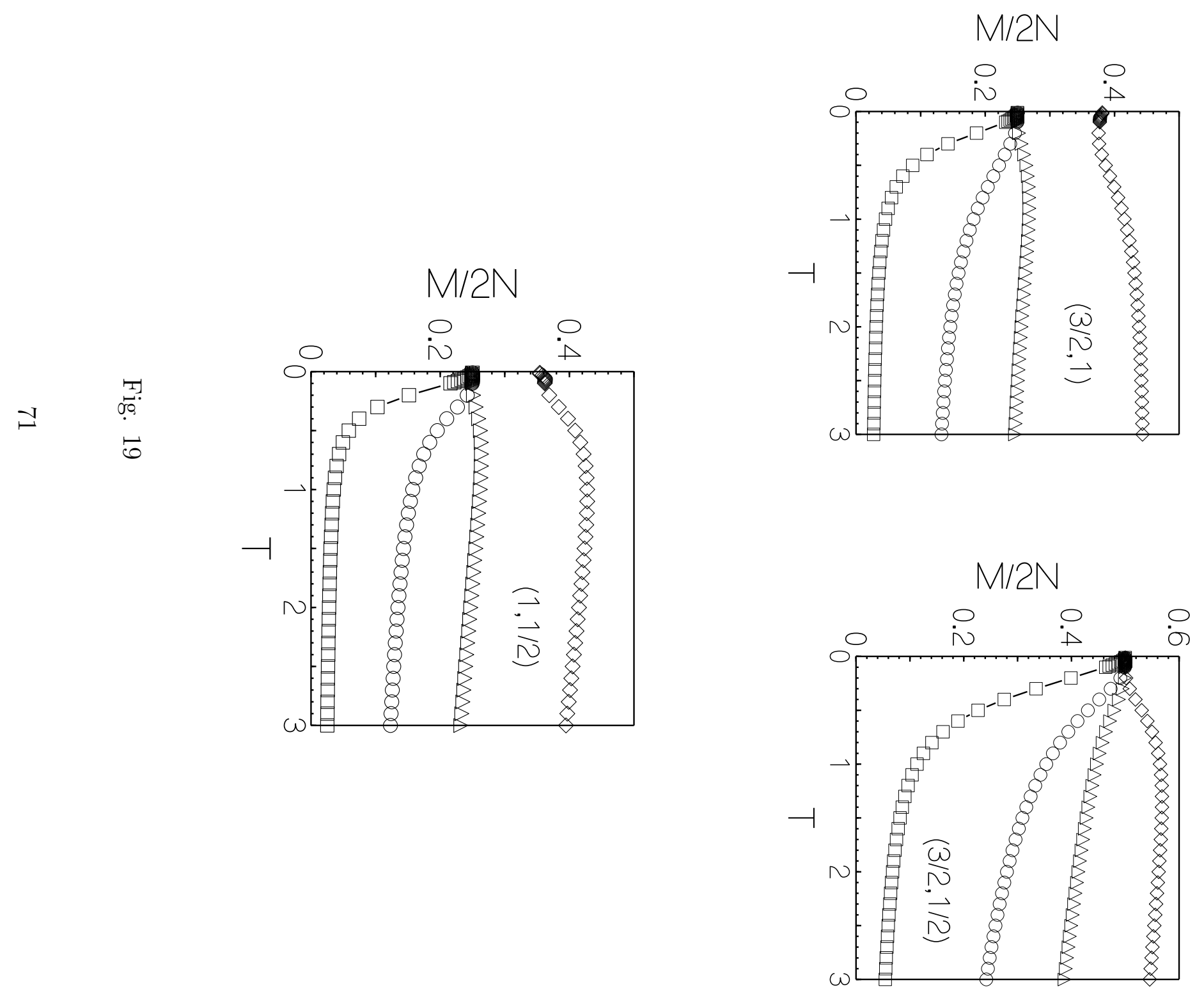




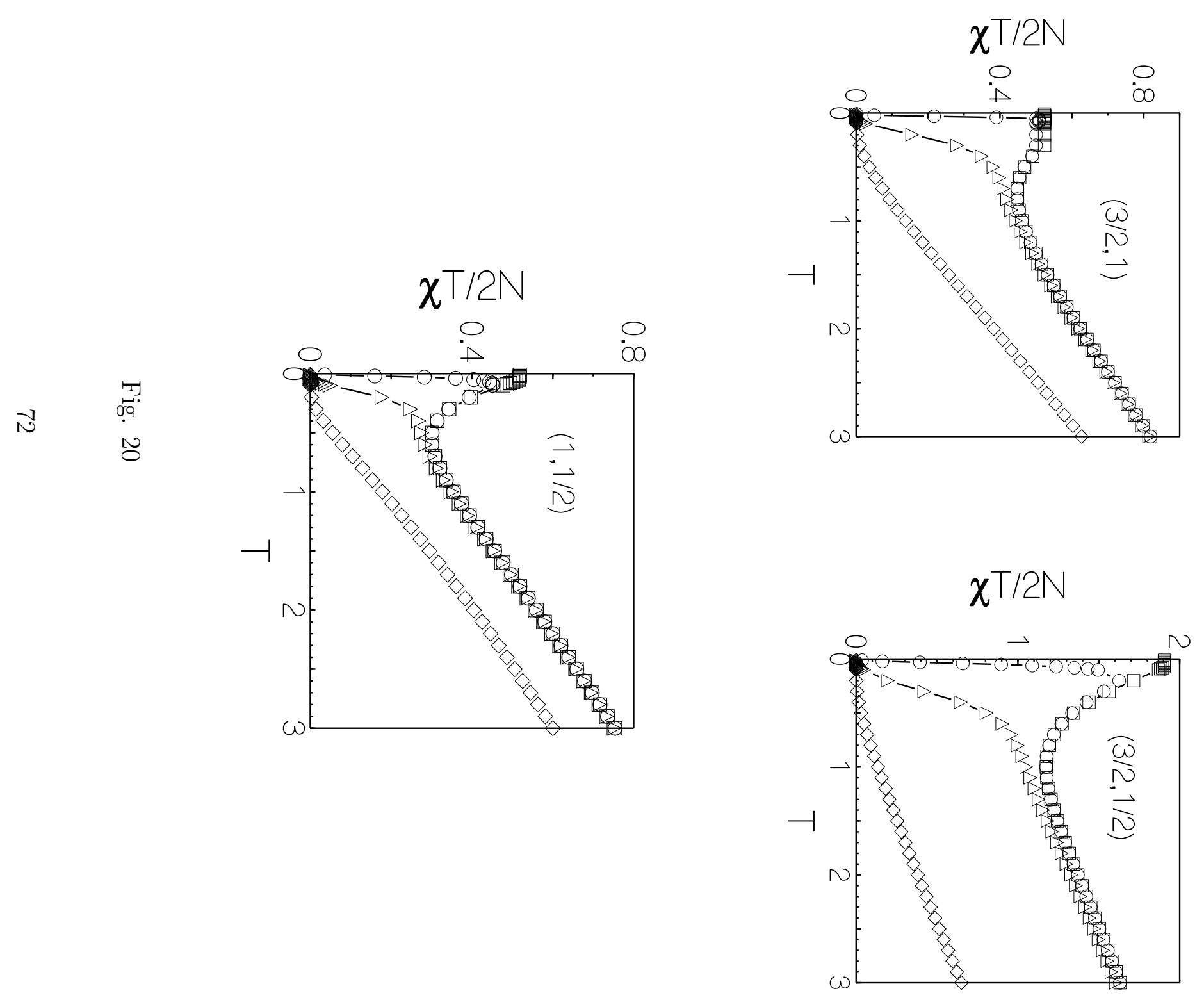




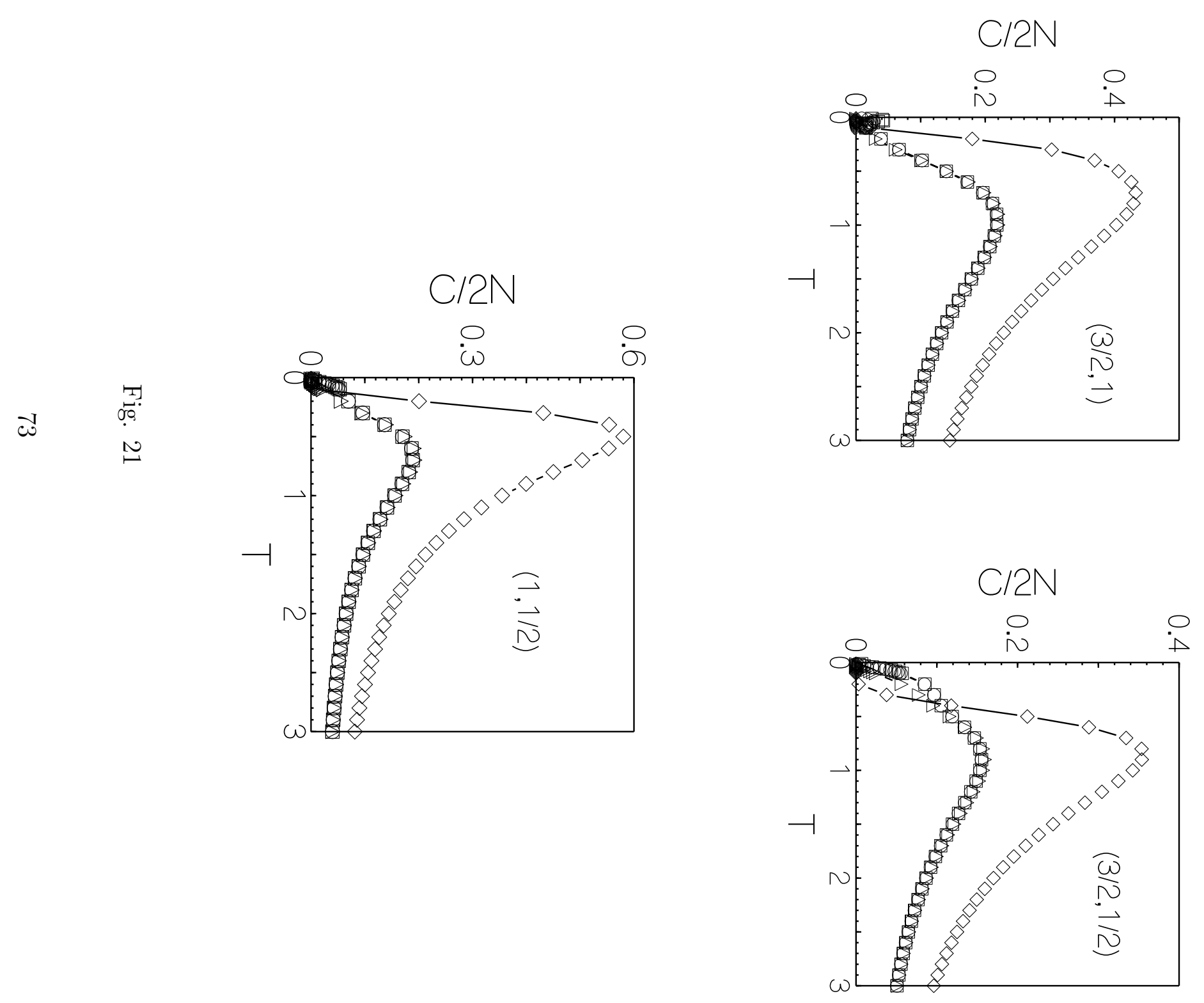




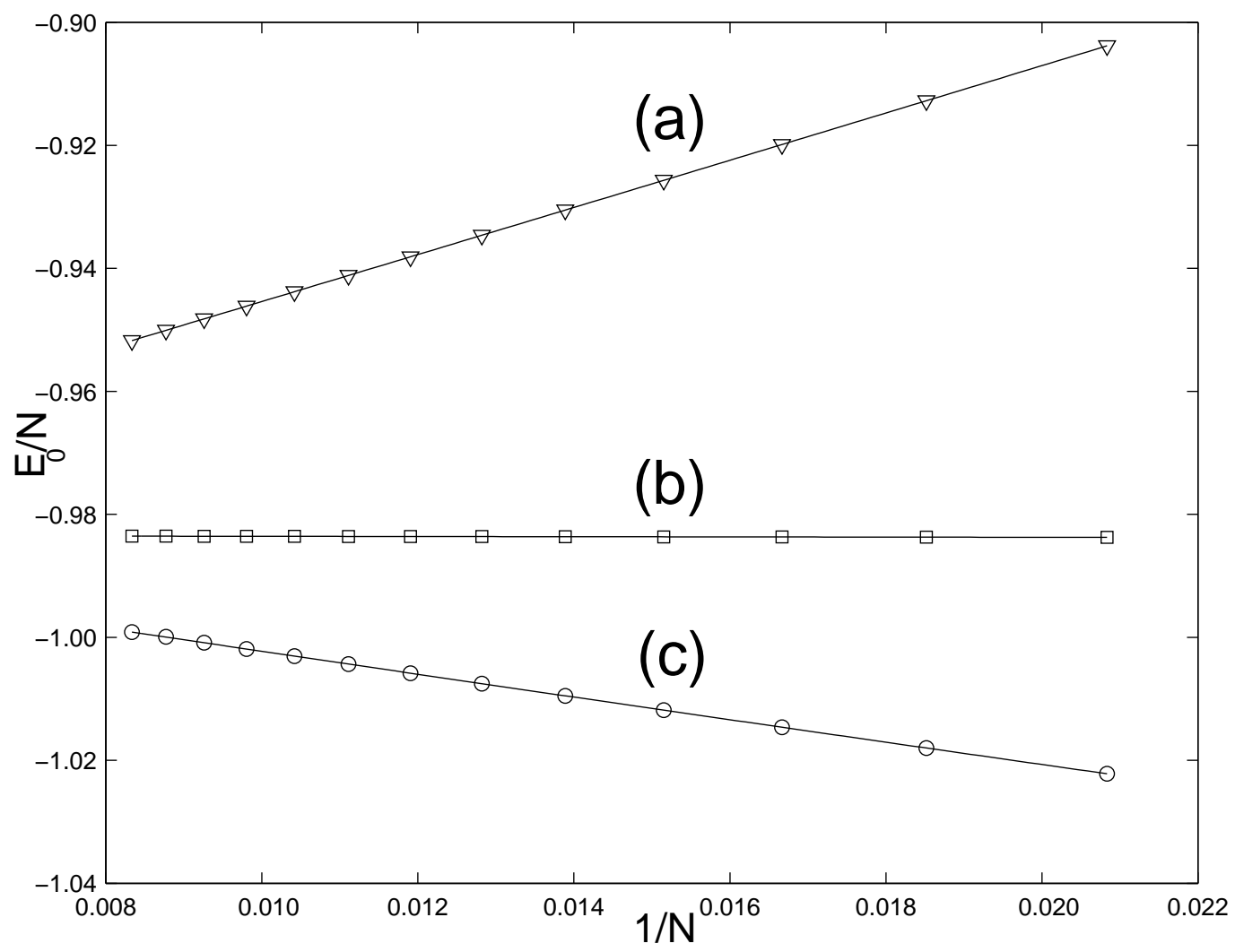

Fig. 22 


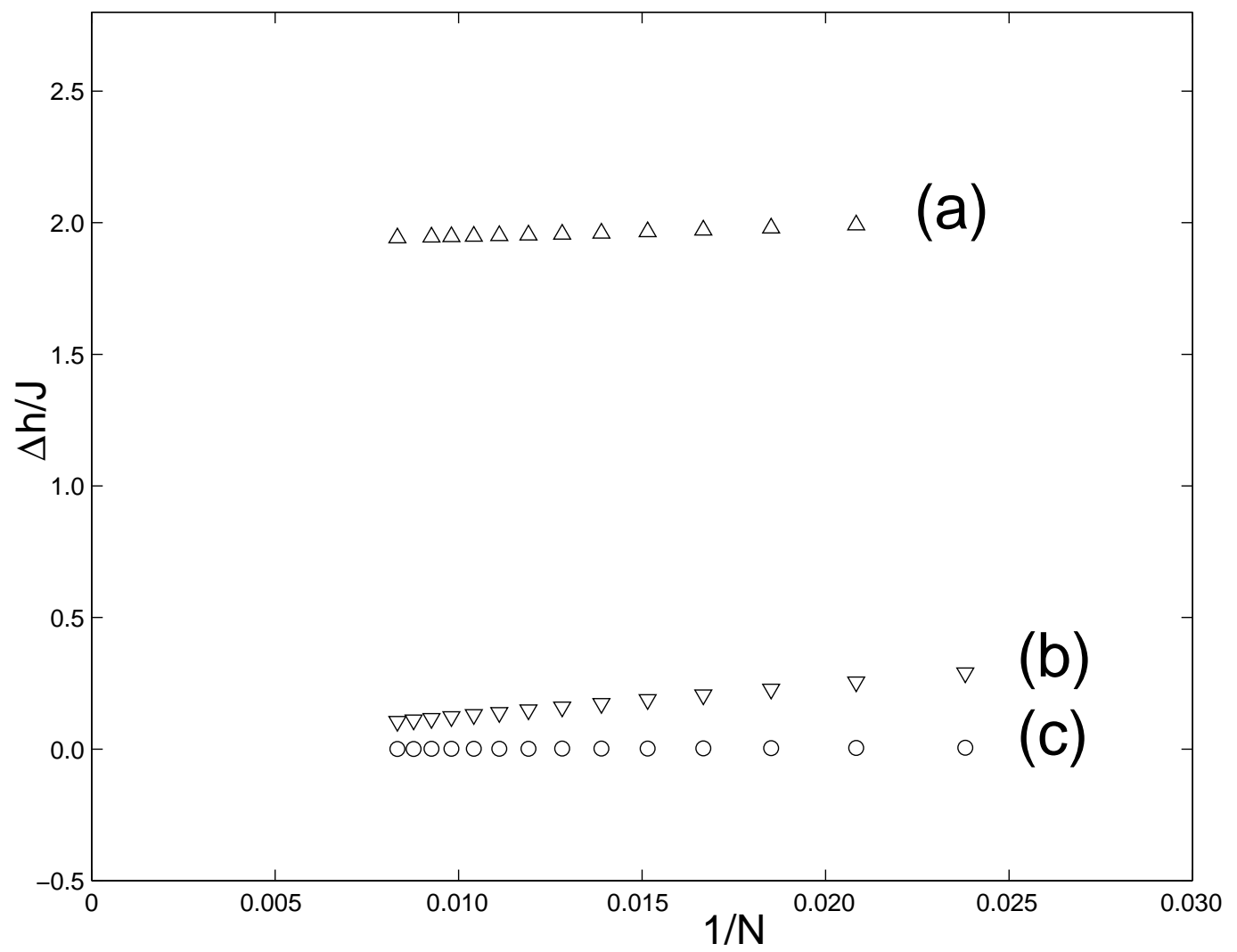

Fig. 23 


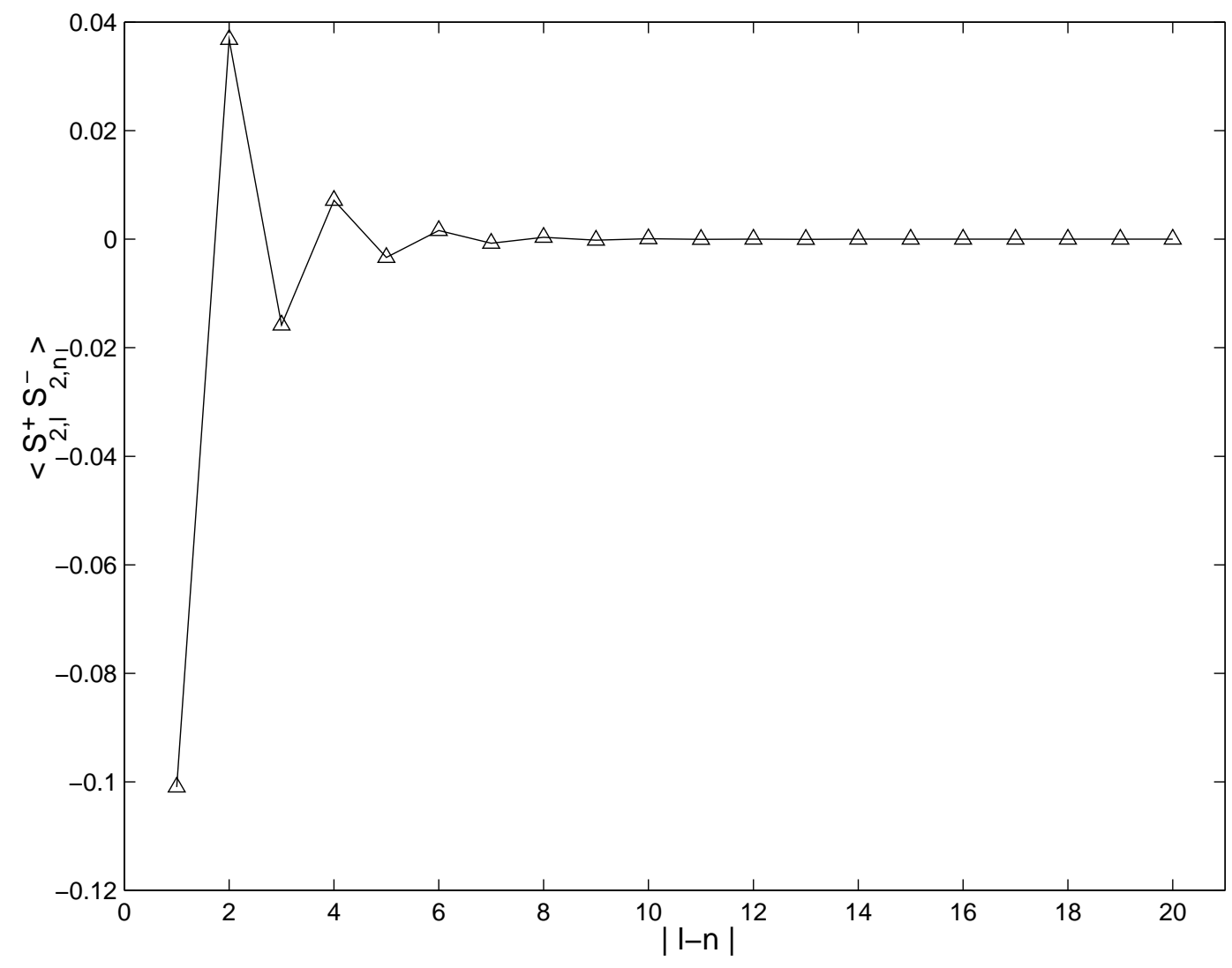

Fig. 24 


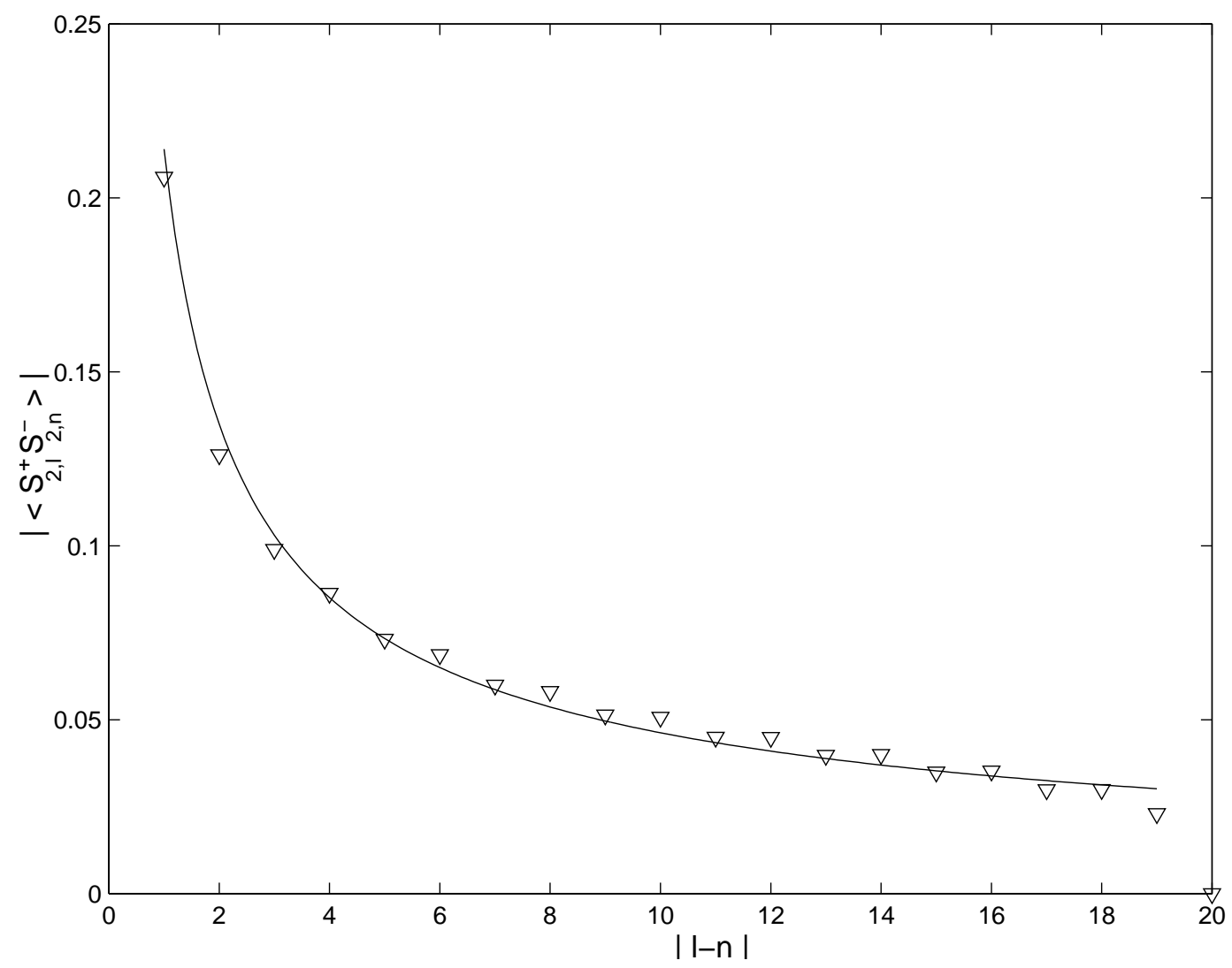

Fig. 25 

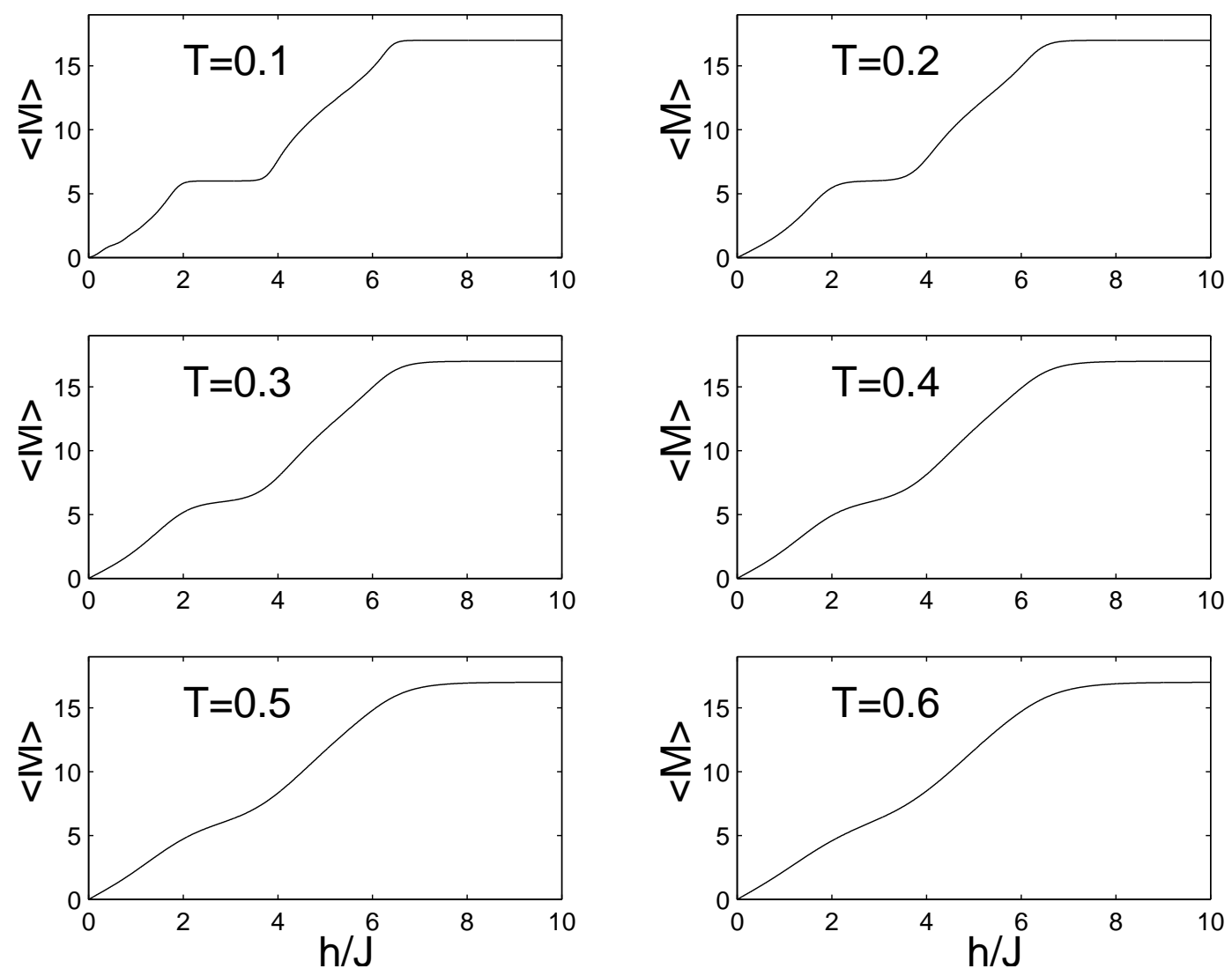

Fig. 26 

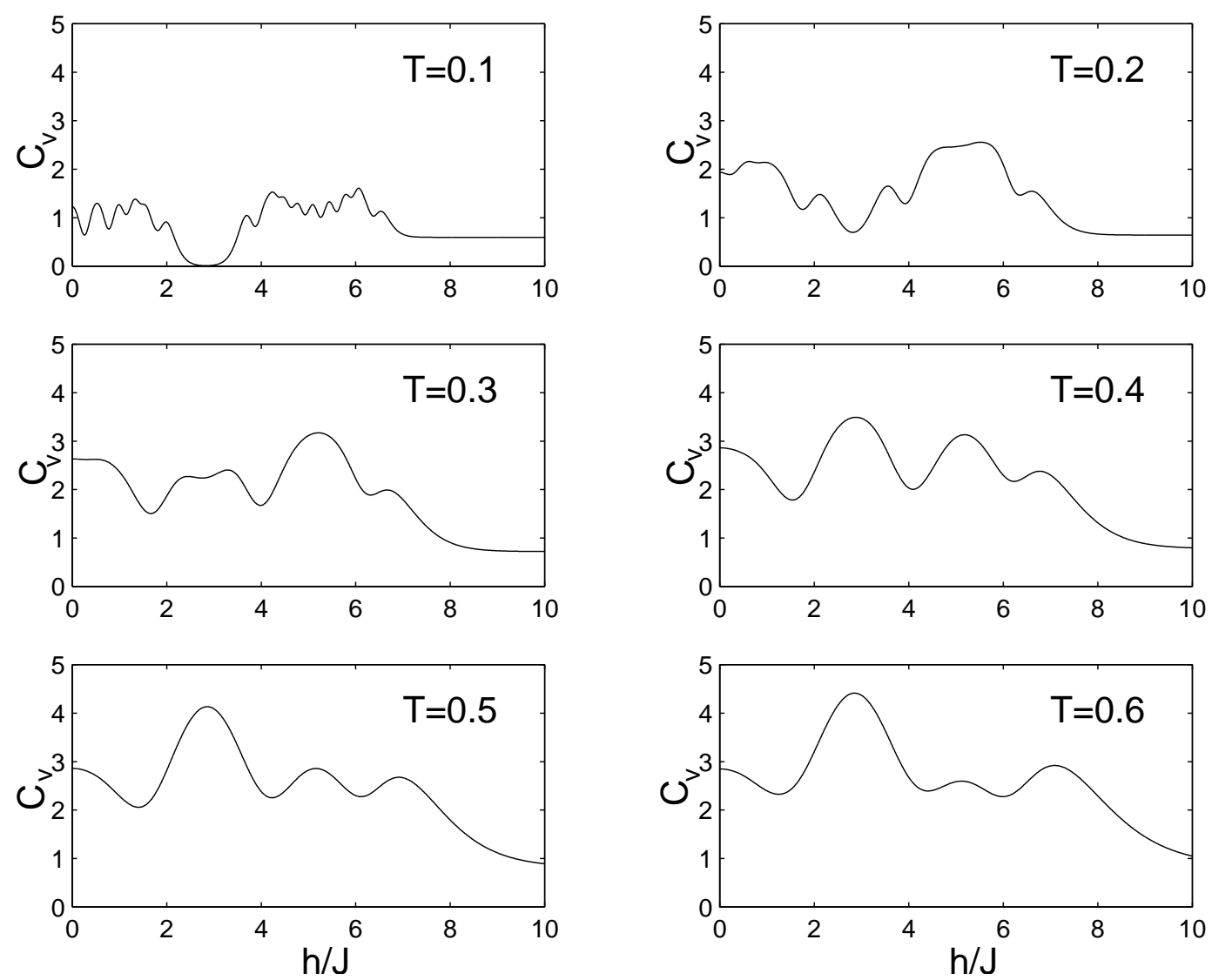

Fig. 27 

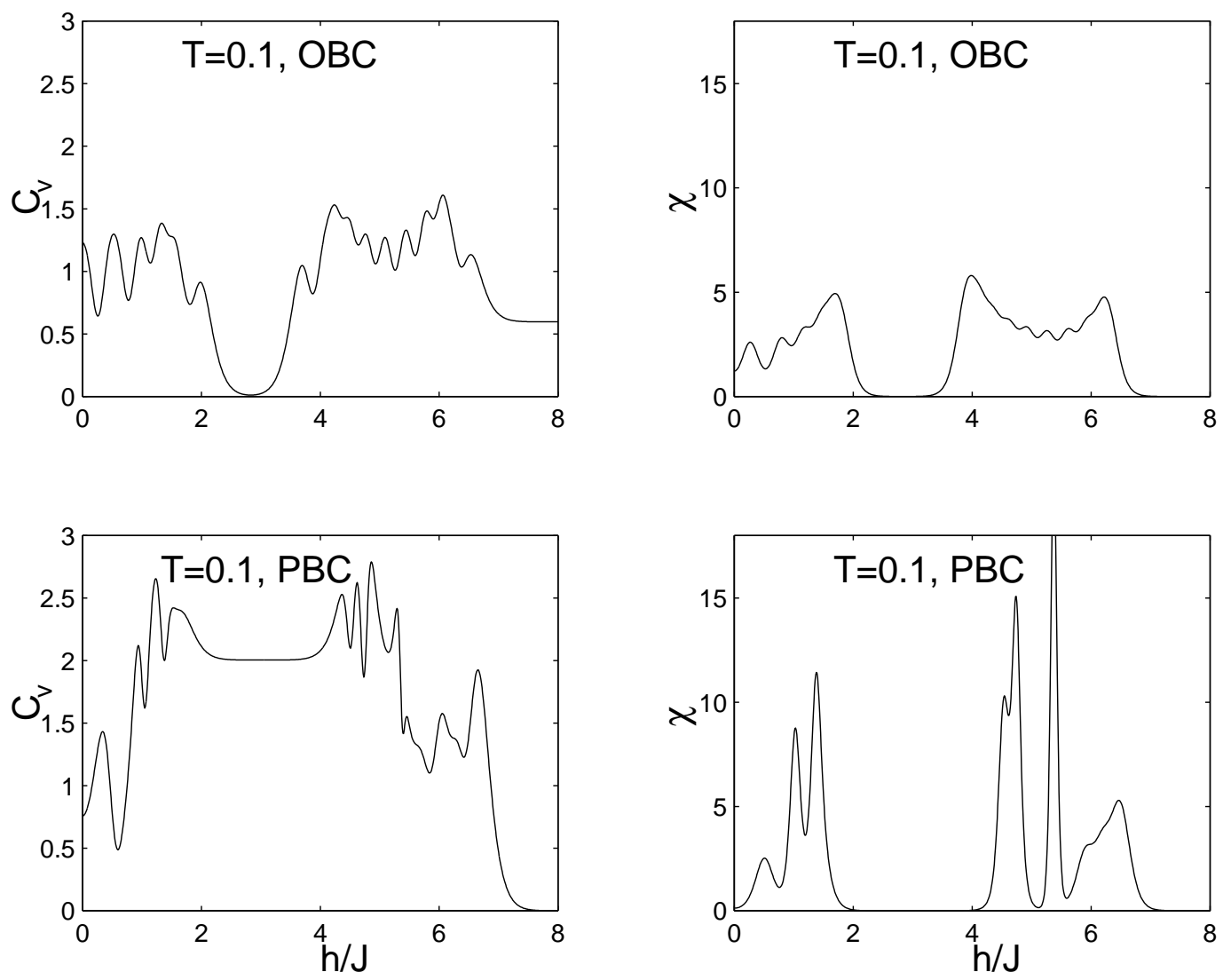

Fig. 28 

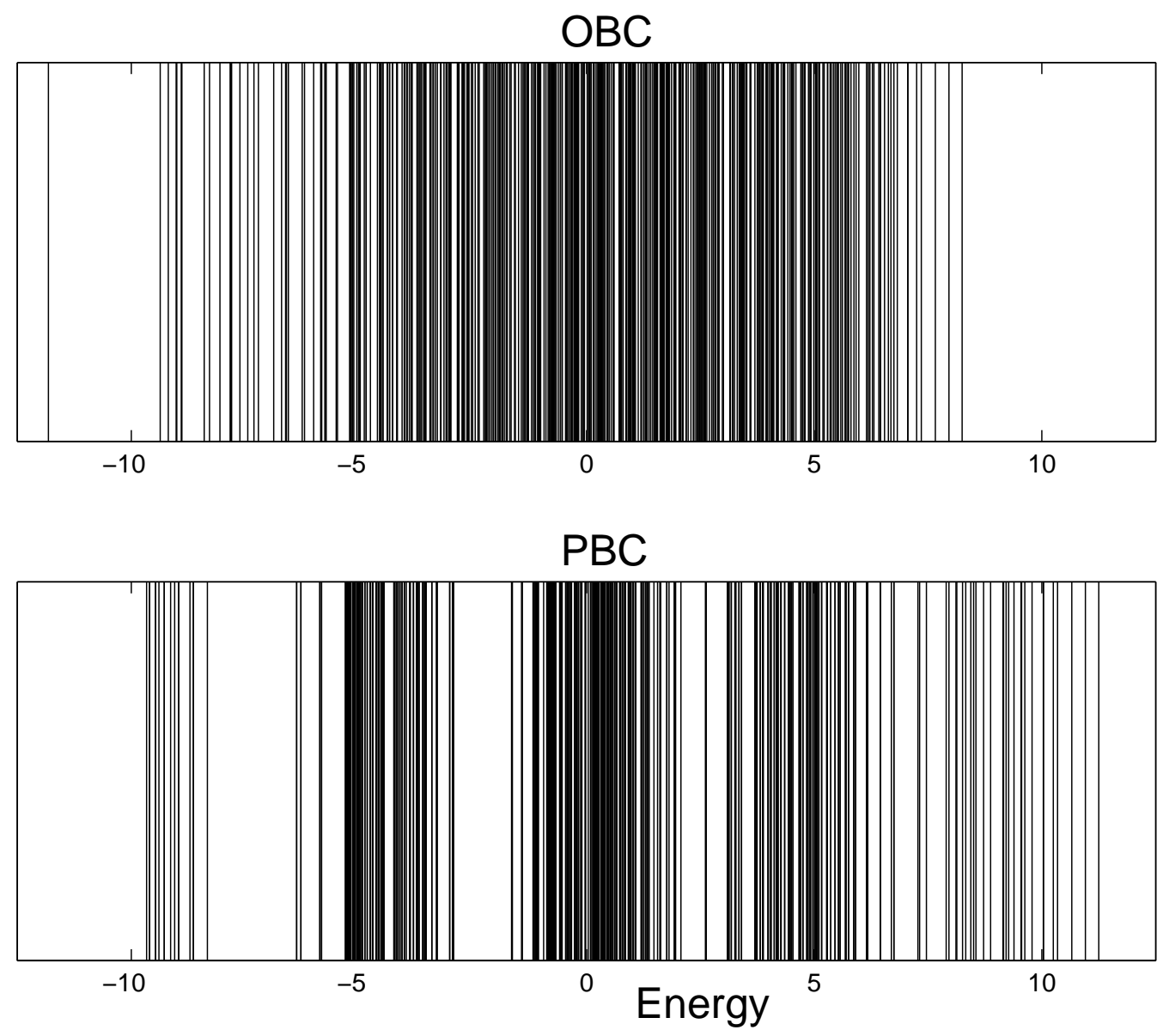

Fig. 29 